AMERICAN FISHERIES SOCIETY

SPECIAL PUBLICATION NO. 201960

SHELVE WITH:

Transactions of the American Eisheries society

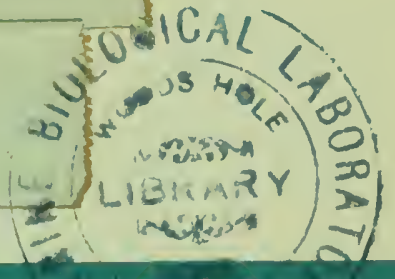

a list of

Common \& Scientific Names of Fishes From the United States \& Canada Second Edition

QL

614

. A43

V. 2 



\section{AMERICAN FISHERIES SOCIETY}

Special Publication No. 2

\section{A List of}

Common and Scientific Names of Fishes from the United States and Canada

( SECOND EDITION)

Report of the Committee on Names of Fishes, Presented at the

Eighty-ninth Annual Meeting, Clearwater, Florida, September 16-18, 1959

Reeve M. Bailey, Chairman

Ernest A. Lachner, C. C. Lindsey, C. Richard Robins

Phil M. Roedel, W. B. Scott, Loren P. Woods

Ann Arbor, Michigan • 1960 
Copies of this publication may be purchased for $\$ 1.00$ each (paper cover) or $\$ 2.00$ (cloth cover). Orders, accompanied by remittance payable to the American Fisheries Society, should be addressed to E. A. Seaman, Secretary-Treasurer, American Fisheries Society, Box 483, McLean, Virginia.

Copyright 1960

American Fisheries Society

Printed by

Waverly Press, Inc.

Baltimore, Maryland 


\section{Introduction}

This second list of the names of fishes of the United States and Canada is not simply a reprinting with corrections, but constitutes a major revision and enlargement. The earlier list, published in 1948 as Special Publication No. 1 of the American Fisheries Society, has been widely used and has contributed substantially toward its goal of achieving uniformity and avoiding confusion in nomenclature. Of the 570 names on that list, 560 are retained in the present version. The 10 removed have since proved to be synonyms of other included species, or are extralimital. In the preparation of the first list, the Committee made arbitrary decisions as to what species should be included as "better known fishes." As interest in fishes expands, it becomes increasingly difficult to determine when a given species merits an approved vernacular or common name. The present Committee has avoided this decision by attempting to include common names for all native and successfully introduced species in the region of coverage. Except for possible errors of oversight, the chief failure to realize complete listing involves small, rare, and poorly-known forms near the periphery of our area: Bering Sea, deepwater fishes that occasionally are found over the continental shelf, and Caribbean species that sporadically invade southern Florida. As knowledge of such forms increases they may be added in subsequent revisions. This list includes 1892 entries, more than a threefold increase. We make no apology for the greater length, believing that these small, less wellknown fishes have real or potential importance as laboratory experimental animals, in public or private aquaria, for bait, or, most often, simply as objects of naturalhistory inquiry or aesthetic appeal. The plan of organization and indexing are such that the inclusion of the lesser fishes need not interfere with use by those not concerned with them.

\section{Area of Coverage}

The present list purports to include all species of fishes known from the fresh waters of the continental United States and Canada, and those marine species inhabiting contiguous shore waters on or above the continental shelf, to a depth of 100 fathoms.
The shore fishes from Greenland, eastern Canada and the United States, and the northern Gulf of Mexico to the mouth of the Rio Grande are included, but those from Iceland, Bermuda, the Bahamas, Cuba and the other West Indian islands, and Mexico are excluded unless they occur also in the region covered. In the Pacific, the area treated includes that part of the continental shelf from the Mexican-United States boundary to Bering Strait. The Arctic shore waters of Alaska and Canada are included. Hawaii, with a large and strikingly different Indo-Pacific fish fauna, is excluded. Deepsea fishes, whether benthic or bathypelagic, are excluded unless they appear also within the 100-fathom isobath. In practice this line of distinction is difficult to apply, and becomes arbitrary to that extent. Pelagic fishes that enter waters over the continental shelf are included. The list provides a general guide to distribution: " $\mathrm{A}$ " denotes Atlantic Ocean but includes the eastern Arctic, "P" refers to Pacific Ocean and includes the western Arctic, and " $F$ " indicates occurrence in fresh water. Appropriate combinations of letters are used for those fishes that live, either naturally or by introduction, in more than one of these regions.

\section{Common Names}

The Committee aims at the development of a body of common names that reflect broad current usage, the creation of a richer, more meaningful and colorful vernacular nomenclature, and the promotion of mechanisms that will add to stability and the universality of names applied to American fishes.

The common name as here employed is viewed as a formal, usually anglicized appellation to be used in lieu of the Latinized scientific name of a species. It is hoped, and the history of the recent past confirms, that common names may be more stable than scientific names. Certainly they should be more readily adaptable to lay uses than scientific names. There is clear need for standardization and uniformity in vernacular names not only for sport or commercial fishes, but as trade names, for aquarium fishes, in legal terminology, and as substitutes for scientific names of almost any fish 
in popular or scientific writing. The Committee believes that it is desirable to establish a common name for each species of fish occurring naturally or through successful introduction in the waters of Englishspeaking North America.

Agreement on many names may be arrived at quickly, but others are attended by complications and marked disagreement develops. This is especially true of fish known by market names that differ from those names that are more familiar to sport fishermen, biologists or others. The existence for a single species of different names in separate parts of its geographic range creates difficulties that seem soluble only through arbitration. Conversely a given name may be employed in several places for diverse species. Although committee action on such situations may not be expected to change local use quickly, at least it seems plainly improper to sanction use of one name for two or more different species.

After wrestling with common names for over twenty years, the Committee on Names of Fishes realized the importance of establishing a set of guiding principles to be employed in the determination of names. Such a code permits a more objective appraisal of the relative merits among several names than if selection is based primarily on personal experience and preference. Consideration of many vernacular names of fishes makes it apparent that few principles can be established for which some exceptions must not be admitted. This is true because at this late date a majority of our larger and more abundant, hence important, species have such firmly established common names that it would be unrealistic to reject them. Decision on the name for a species may often be decided by weighing the pros and cons among the possible alternatives and selecting that one which best fits the aggregate of guiding criteria.

In the introduction to the earlier list of names a number of rules of procedure were itemized. Recently the Committee incorporated these into the following statement of criteria that it regards as appropriate to the selection of common names of fishes.

\section{Principles Governing Selection of Common Names ${ }^{1}$}

1. A single vernacular name shall be accepted for each species or taxonomic unit included.-

\footnotetext{
1 First printed in 1955 (Trans. Am. Fish. Soc., Vol. 84 [1954] pp. 368-371).
}

In the present list only two fishes have two approved names: redfish and ocean perch for Sebastes marinus; cisco and lake herring for Coregonus artedii.

2. No two species on the list shall have the same approved name.

3. The expression "common" as part of a fish's name shall be avoided wherever possible.

4. Simplicity in names is favored.-Hyphens, suffixes, and apostrophes shall be omitted (e.g., smallmouth bass) except where they are orthographically essential (e.g., threeeye flounder), have a special meaning (e.g., C-O sole), or are necessary to avoid possible misunderstanding (e.g., cusk-eel). Compounded modifying words, including paired structures, should usually be treated as singular nouns in apposition with a group name (e.g., spottail shiner, soupfin shark), but a plural modifier should usually be placed in adjectival form (e.g., blackbanded sunfish, spotted hake). Preference shall be given to names that are short and euphonious.

The compounding of brief, familiar words into a single name, written without hyphen, may in some cases promote clarity and simplicity (e.g., tomcod, goldfish, mudminnow), but the wholesale practice of combining words, especially those that are lengthy, awkward, or unfamiliar, is deplored.

5. Common names shall not be capitalized in text use except for those elements that are proper names (e.g., rainbow trout but Sacramento perch).

6. Names intended to honor persons (e.g., Allison's tuna, Julia's darter) are not admissible.

7. Only clearly defined and well-marked taxonomic entities (usually species) shall be assigned common names.-Most subspecies are not suitable subjects for common names, but those forms that are so different in appearance (not just in geographic distribution) as to be distinguished readily by sport or commercial fishermen or laymen or for which a common name constitutes a significant aid in communication may merit separate names. Subspecies have importance in evolutionary inquiry but are rarely of significance to laymen or in those aspects of biological endeavor in which common names are of concern. The practice of adding geographic modifiers to designate regional 
populations makes for a cumbersome terminology.

8. The common name shall not be intimately tied to the scientific name. - Thus the vagaries of scientific nomenclature do not entail constant changing of common names. The practice of applying a name to each genus, a modifying name for each species, and still another modifier for each subspecies, while appealing in its simplicity, has the defect of inflexibility. If a fish is transferred from genus to genus, or shifted from species to subspecies or vice versa, the common name should nevertheless remain unaffected. It is not a primary function of common names to indicate relationship. When two or more taxonomic groups (e.g., nominal species) are found to be identical, one name shall be adopted for the combined group.

This principle is regarded not only as fundamental to the achievement of stability, but as essential to the development of a true vernacular nomenclature.

9. Names shall not violate the tenets of good taste.

The preceding principles are largely in the nature of procedural dicta. Those given below are criteria that are regarded as aids in the selection of suitable names.

10. Colorful, romantic, fanciful, metaphorical, and otherwise distinctive and original names are especially appropriate.-Such terminology adds to the richness and breadth of the nomenclature and yields a harvest of satisfaction to the user. Examples of such names include: Dolly Varden, madtom, hogchoker, garibaldi, pumpkinseed, flier, angelfish, midshipman, and chilipepper.

11. American Indian names are welcome for adoption as common names. -Those in current use include muskellunge, eulachon, mummichog, chinook, tautog, menhaden, and cisco.

12. Regardless of origin, truly vernacular names that are widespread and in common use by the public are to be retained wherever possible.-In addition to aboriginal names, many now well-known names of American fishes have been derived from non-Englishspeaking fishermen of foreign extraction: barracuda, cero, grouper, pompano (Spanish); bocaccio (Italian); capelin, inconnu (French). Although too little genuine originality is evident, many excellent names have been developed by American immigrants. Most of these conform to principles 14 and 15 below.

13. Commonly employed names adopted from traditional English usage (e.g., cod, pike, sole, flounder, bass, perch, chub, minnow) may be given considerable latitude in taxonomic placement.-Adherence to customary English practice is to be preferred if this does not conflict with the broad general use of another name. Many English names, however, have been applied to similar appearing but often distantly related fishes in America. We find perch in use for representatives of at least 9 families of spinyrayed fishes. Chub appears in such diverse groups as the Salmonidae, Cyprinidae, and Kyphosidae. The ocean whitefish (Caulolatilus princeps) is not a salmonid and the blue pike (Stizostedion vitreum glaucum) is remote from the Esocidae, yet each is best known to fishermen throughout its range by the name indicated. For widely-known species the Committee believes it preferable to recognize general use than to engage in a probably futile effort to gain the general adoption of bookish or pedantic substitutes. Thus, established practice should outweigh consistency with original English usage.

14. Structural attributes, including color and color pattern, are desirable and are in common use in forming names. - Sailfin, flathead, slippery, giant, mottled, copper, tripletail, and a multitude of others decorate fish names. Efforts should be made to select terms that are descriptively accurate, and to hold repetition of those most frequently employed (e.g., white, black, spotted, banded) to a minimum.

15. Ecological characteristics are useful in making good names. - They too should be properly descriptive. Terms such as sand, rock, lake, riffle, freshwater and mountain are well known in fish names.

16. Geographic distribution provides suitable adjectival modifiers. - Poorly descriptive or misleading geographic characterizations (e.g., Kentucky bass for a wide-ranging species) should be corrected unless they are too deeply entrenched in current usage. In the interest of brevity it is usually possible to delete words such as lake, river, or ocean in the names of species (e.g., Colorado squawfish, not Colorado River squawfish).

17. Generic names may be employed outright (e.g., remora) or in modified form (e.g., 
molly, from Mollienesia) as common names.Once adopted such names should be maintained even if the generic name is changed. These vernaculars should be written in Roman and without capitalization. Brevity and euphony are of especial importance for names of this type, which will probably be adopted most often for aquarium fishes or other smaller and little-known kinds that do not already have a well-established vernacular nomenclature.

18. The duplication of common names for fishes and other organisms should be avoided if possible, but names in wide general use need not be rejected on this basis alone.-The name dolphin is commonly applied to certain cetaceans and also to Coryphaena hippurus. Similarly, buffalo is employed for various artiodactyls and for suckers of the genus Ictiobus. On the basis of prevailing use these names are admissible as fish names.

19. Names that appear on official lists of names of fishes prepared by other agencies shall be preferred.

\section{PlaN of List}

The list is presented as a natural or phyletic sequence of families of fishes, with the species of each family alphabetized to generic and specific names. In order to facilitate use the three classes of recent fishes (Agnatha-jawless fishes, Chondrichthyescartilaginous fishes, and Osteichthyes-bony fishes) and the orders are indicated. For the latter, the ordinal names of the Goodrich system (formes endings) are given first, followed, in parentheses, by equivalent names employed by Regan, Jordan, Romer or others. Both common and technical names of families (idae endings) are given. Within families the sequence is alphabetical to scientific name; the occasional disadvantage of separating closely related forms within a family is regarded as more than offset by the greater ease in use.

An innovation in this edition is the inclusion of authorities for scientific names. These are commonly needed by fishery workers, who may not have ready access to the necessary references. In any event their appearance here in a single cover is a great convenience. Care has been taken to prevent errors in author's names; usage here reflects current interpretation of the International Rules on Zoological Nomenclature. In line with those rules, the author's name(s) follows the specific name directly and without punctuation if the species, when originally described, was assigned to the same genus in which it appears here; if the species was described in another genus, the author's name(s) appears in parentheses. For example, the brook trout was originally named Salmo fontinalis by Mitchill; it appears here as Salvelinus fontinalis (Mitchill).

In preparation of the list hundreds of decisions had to be made that involved interpretation of rules of nomenclature, systematic status and limits of species, genera, and families, occurrence within the prescribed region, and other problems. Four times in six years it has been possible for a majority of the committee members to gather around a table and attack these problems together. The resultant interchange of ideas and information, extended by circulation of dozens of work lists, has made possible striking concordance on what started as areas of disagreement. There remain, to be sure, many differences of opinion, but there has been a broadened area of understanding and a spirit of good humor and willingness to compromise difficulties. Decisions have been made by majority opinion of the Committee. Thus, no committee member subscribes to all decisions reached. In many places, information available to the Committee exceeded that in the current literature. In general it has been our policy to withhold making such changes here, although we have made some considered exceptions, especially when the new data will appear shortly.

\section{INDICES}

The main list, Part I, appears on pages 6 to 50 ; there follow indices, Part II, to common names (pages 51 to 75) and, Part III, to scientific names (pages 76 to 102 ). The index to common names gives page references to approved common names of classes, families, and species. A single entry is included for each species; for example, Salmo gairdneri is entered only under "trout, rainbow." In addition to approved names, the index includes also other names that are in wide use followed by the equivalent approved name. There are a great many vernacular names of American fishes, and extensive search would be necessary to assemble even a large fraction of them. This the Committee has not attempted to do, but most of the more widely used names are included.

The index to scientific names gives page references to the names here entered for 
classes, orders, families, genera, and species. Each species is entered only under its specific name. For example, the bluegill may be located under "macrochirus, Lepomis" but not under Lepomis macrochirus, although an entry for Lepomis directs the reader to the proper page. Scientific names that are not accepted in this list are excluded except for those that appeared in the first edition and have since been placed in synonymy.

\section{ACKNowledgments}

Many persons have contributed to the preparation of this report. In addition to the present Committee, these include author's of the first edition, Walter A. Chute, W. A Clemens, John R. Dymond, the late Samuel F. Hildebrand, George S. Myers, and Leonard P. Schultz, and others who served during the interim between the two editions, Charles M. Breder, Ralph Hile, Raymond E. Johnson, Frank T. Knapp, John C. Marr, Edward C. Raney, William E. Ricker, and Royal D. Suttkus. Specialists have been most generous in their assistance, which is much appreciated. Among the many, a few may be mentioned for their especial helpfulness: Frederick H. Berry, James E. Böhlke, John C. Briggs, Homer Circle, Daniel M.
Cohen, John E. Fitch, W. I. Follett, Earl S. Herald, Carl L. Hubbs, Clark Hubbs, William A. Kennedy, A. H. Leim, Gordon F. Luce, Jr., IVilliam R. Martin, Robert R. Miller, George A. Moore, C. Lavett Smith, William R. Taylor, Boyd W. Walker, Vladimir Walters, and Norman J. Wilimovsky.

In 1951 the American Fisheries Society approved a plan whereby the Conmittee on Names of Fishes of that Society would serve jointly as a Committee on Common Names of Fishes in the American Society of Ichthyologists and Herpetologists. The president of the ASIH formally appointed the Committee. Since that time this arrangement has worked smoothly. All personnel on the Committee are members of both societies and the formulation of this list, it is believed, serves common interests. Annual meetings of the ASIH have provided a convenient opportunity to assemble the joint committee to attack problems in the preparation of this list. The plan is mutually advantageous, and we hope it may continue.

We gratefully acknowledge financial assistance in the publication of this list provided by the National Science Foundation. (NSF-G12424). 


\section{PART I}

\section{Accepted Common Name, Occurrence, and Scientific Name}

COMMON NAME $\quad$ OCCURRENCE ${ }^{1}$ SCIENTIFIC NAME

\section{CLASS AGNATHA-JAWLESS FISHES \\ Order Myxiniformes (Myxinoidea; Hyperotreti) \\ Myxinidae-hagfishes}

Atlantic hagfish............. A ............. Myxine glutinosa Linnaeus Black hagfish............... P ..Polistotrema deani Evermann and Goldsborough Pacific hagfish .............. P . . . . . . . Polistotrema stouti (Lockington)

Order Petromyzontiformes (Petromyzontia; Hyperoartit)

Petromyzontidae-lampreys

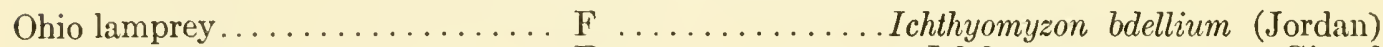
Chestnut lamprey............F . . ........... Ichthyomyzon castaneus Girard Northern brook lamprey ......... F ..... Ichthyomyzon fossor Reighard and Cummins Southern brook lamprey........F . ...... Ichthyomyzon gagei Hubbs and Trautman Allegheny brook lamprey........ F ..... Ichthyomyzon greeleyi Hubbs and Trautman Mountain brook lamprey........F ............... Ichthyomyzon hubbsi Raney Silver lamprey.............. F ...Ichthyomyzon unicuspis Hubbs and Trautman

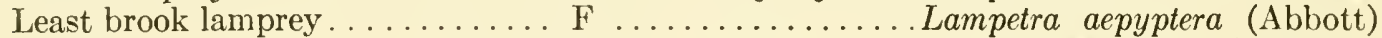

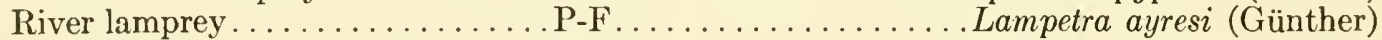
Arctic lamprey ............. .F. . . . . . . . . Lampetra japonica (Martens)

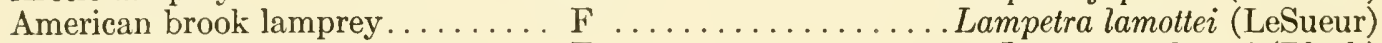

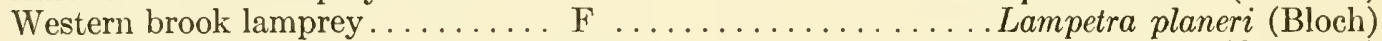
Pacific lamprey ..............F. . . . . . . . . Lampetra tridentata (Gairdner)

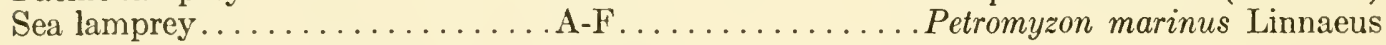

\section{CLASS CHONDRICHTHYES-CARTILAGINOUS FISHES}

\section{Order Squaliformes (Selachit)}

Hexanchidae-cow sharks

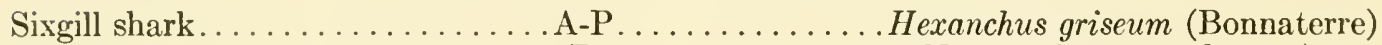

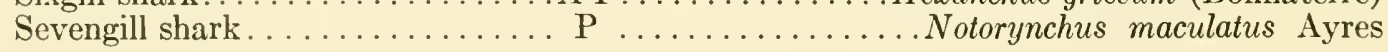

Chlamydoselachidae-frill sharks

Frill shark. ............. . ....... Chlamydoselachus anguineum Garman

Heterodontidae-horn sharks

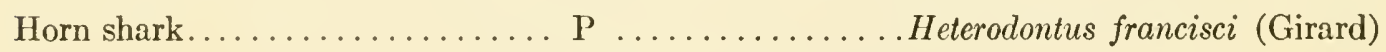

Carchariidae-sand sharks

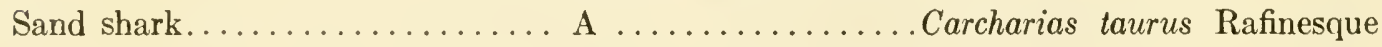

Lamnidae-mackerel sharks

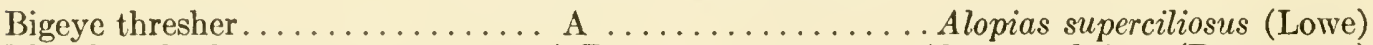

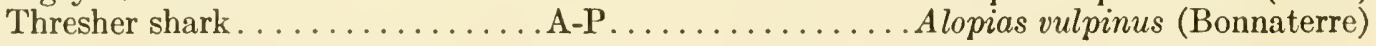

$\therefore=$ Atlantic; $P=$ Pacific; $F=$ Freshwater 
White shark.

A-P.

Carcharodon carcharias (Linnaeus)

Basking shark.............. A-P

.Cetorhinus maximus (Gunnerus)

Bonito shark

Mako.

Salmon shark.

Lamna nasus (Bonnaterre)

Orectolobidae - nurse sharks

Nurse shark. . Ginglymostoma cirratum (Bonnaterre)

Rhincodontidae-whale sharks

Whale shark. A-P Rhincodon typus Smith

Scyliorhinidae-cat sharks

Brown cat shark............ P

Swell shark................ P

Filetail cat shark............ P

False cat shark ............ A

Chain dogfish.............. A
Apristurus brunneus (Gilbert) Cephaloscyllium uter (Jordan and Gilbert) Parmaturus xaniurus (Gilbert) A .........Pseudotriakis microdon Brito Capello A .................. Scyliorhinus retifer (Garman)

\section{Carcharhinidae-requiem sharks}

Finetooth shark.

Blacknose shark.

Bignose shark.

Roundnose shark

Sickle shark

Silky shark.

Bay shark.

A .........Aprionodon isodon (Müller and Henle)

A

Bull shark.

Blacktip shark

Whitetip shark.

Spinner shark.

Sandbar shark

Dusky shark.

Smalltail shark.

Narrowtooth shark ............ P Tiger shark.

Soupfin shark

Night shark.

Gray smoothhound

Smooth dogfish

Sicklefin smoothhound

Florida smoothhound

Lemon shark.

Blue shark.

Pacific sharpnose shark . . . . . . A-P

Ptlantic sharpnose shark........ P

Brown smoothhound.......... P

Leopard shark.
A

A

A

A

A

A

A

A-P $\mathrm{P}$ A

$\mathrm{P}$

A

A

A $\mathrm{P}$

Carcharhinus acronotus (Poey)

. Carcharhinus altima (Springer)

Carcharhinus azureus (Gilbert and Starks) . Carcharhinus falciformis (Müller and Henle) . Carcharhinus floridanus Bigelow,

Schroeder, and Springer .Carcharhinus lamiella (Jordan and Gilbert) Carcharhinus leucas (Müller and Henle) . Carcharhinus limbatus (Müller and Henle) .Carcharhinus longimanus (Poey) Carcharhinus maculipinnis (Poey) . Carcharhinus obscurus (LeSueur) . Carcharhinus porosus (Ranzani) . Carcharhinus remotus (Duméril) Galeocerdo cuvieri (Peron and LeSueur) Galeorhinus zyopterus Jordan and Gilbert Hypoprion signatus Poey Mustelus californicus Gill Mustelus canis (Mitchill) Mustelus lunulatus Jordan and Gilbert .Mustelus norrisi Springer Negaprion brevirostris (Poey) Prionace glauca (Linnaeus) ......... Scoliodon longurio (Jordan and Gilbert) A .............Scoliodon terraenovae (Richardson) P .................... Triakis henlei (Gill) P .................. Triakis semifasciata Girard Carcharhinus milberti (Müller and Henle)

Sphyrnidae-hammerhead sharks

Scalloped hammerhead ............A-P. . Sphyrna lewini (Griffith) Great hammerhead. 
Bonnethead.

A-P

Sphyrna tiburo (Linnaeus)

Smooth hammerhead.

A-P Sphyrna zygaena (Linnaeus)

Squalidae-dogfish sharks

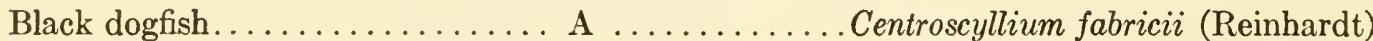

Kitefin shark............... A Dalatias licha (Bonnaterre)

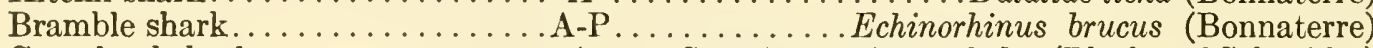

Greenland shark ............. A ....Somniosus microcephalus (Bloch and Schneider)

Pacific sleeper shark........... P ......Somniosus pacificus Bigelow and Schroeder

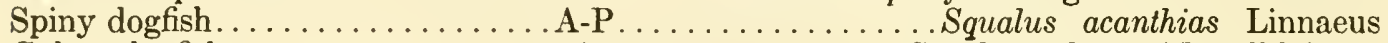

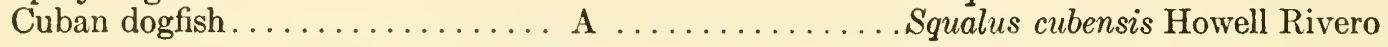

Squatinidae -angel sharks

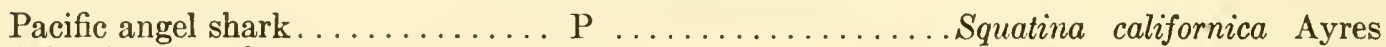

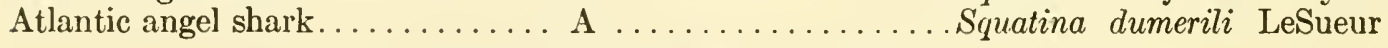

\section{Order Rajiformes (Batoidei)}

Pristidae-sawfishes

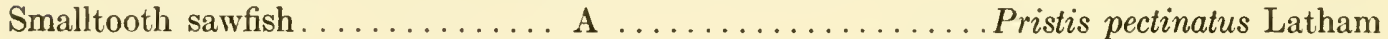

Largetooth sawfish

Rhinobatidae-guitarfishes

Thornback ............. P ...Platyrhinoidis triseriata (Jordan and Gilbert)

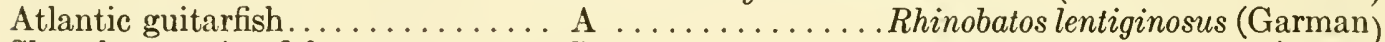

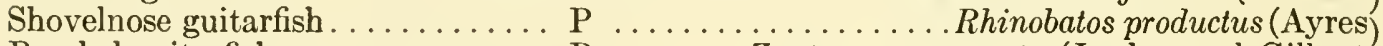
Banded guitarfish ............ P ....... Zapteryx exasperata (Jordan and Gilbert)

Torpedinidae-electric rays

Lesser electric ray............. A

Pacific electric ray. . . . . . . . . P

Atlantic torpedo.
Narcine brasiliensis (Olfers)

Rajidae-skates

Ocellate skate.............. A

Aleutian skate............... P

Big skate. . . ............ P

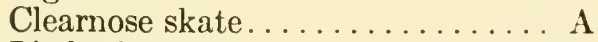

Little skate. . . . . . . . . . . . . A A

Rosette skate............... A

California skate.............. P

Bering skate.............. P

Black skate............... P

Barndoor skate................ A

Freckled skate.............. A

Winter skate................ A

Spreadfin skate.............. A

Alaska skate................. P

Thorny skate................ A

Longnose skate.............. P

Flathead skate............... P

Smooth skate. Torpedo californica Ayres Torpedo nobiliana Bonaparte

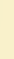
(

$\mathrm{A}$
$\mathrm{P}$
$\mathrm{A}$
$\mathrm{P}$
$\mathrm{P}$
$\mathrm{P}$
$\mathrm{A}$
$\mathrm{A}$
$\mathrm{A}$
$\mathrm{A}$
$\mathrm{P}$
$\mathrm{A}$
$\mathrm{P}$
$\mathrm{P}$
$\mathrm{A}$
Raja ackleyi Garman Raja aleutica Gilbert Raja binoculata Girard Raja eglanteria Bosc Raja erinacea Mitchill Raja garmani Whitley Raja inornata Jordan and Gilbert Raja interrupta Gill and Townsend Raja kincaidi Garman . . . . . . . Raja lentiginosa Bigelow and Schroeder Raja ocellata Mitchill . . . . . . . Raja olseni Bigelow and Schroeder Raja parmifera Bean $\ldots \ldots \ldots$. . . Raja rhina Jordan and Gilbert Raja rosispinis Gill and Townsend Raja senta Garman 
Spinytail skate.

Starry skate.

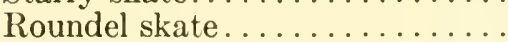

Roughtail skate.
A $\mathrm{P}$

A

$\mathrm{P}$

\begin{abstract}
Raja spinicauda Jensen Raja stellulata Jordan and Gilbert Raja texana Chandler Raja trachura Gilbert
\end{abstract}

Dasyatidae - stingrays

Southern stingray.

A ....Dasyatis americana Hildebrand and Schroeder

Roughtail stingray A

Diamond stingray $\mathrm{P}$

Atlantic stingray...

A

Bluntnose stingray

A

Pelagic stingray....

Spiny butterfly ray

Smooth butterfly ray.

Round stingray. .Dasyatis centroura (Mitchill)

Dasyatis dipterurus (Jordan and Gilbert) Dasyatis sabina (LeSueur)

Yellow stingray.............

Spotted eagle ray.

Bat stingray.

Bullnose ray

Southern eagle ray . . . . . . . .

Cownose ray. . . . . . . . . . . . . .
$\mathrm{P}$

A

$\mathrm{P}$

A

$\mathrm{P}$

A

Myliobatidae - eagle rays
A
$\mathrm{P}$
A
A
A
Aetobatus narinari (Euphrasen)
...Myliobatis californicus Gill
$\ldots \ldots \ldots \ldots \ldots$ Myliobatis freminvillei LeSueur

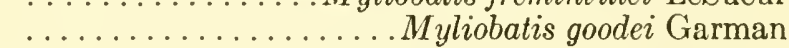
................. Rhinoptera bonasus (Mitchill)

Dasyatis violacea (Bonaparte)

Gymnura altavela (Linnaeus)

Gymnura marmorata (Cooper)

....... Gymnura micrura (Bloch and Schneider)

Urolophus halleri Cooper

Urolophus jamaicensis (Cuvier)

Mobulidae-mantas

Atlantic manta

A

Pacific manta................ P

Devil ray................. A

Spinetail mobula............ P

Smoothtail mobula........... P

Manta birostris (Walbaum)

Manta hamiltoni (Newman)

Mobula hypostoma (Bancroft)

Mobula japanica (Müller and Henle)

Mobula lucasana Beebe and Tee-Van

\section{Order Chimaeriformes (Chimaerae)}

Chimaeridae-chimaeras

Ratfish.

$P$

Hydrolagus colliei (Lay and Bennett)

\section{CLASS OSTEICHTHYES-BONY FISHES}

\section{Order ACipenseriformes (Chondrostei)}

Acipenseridae-sturgeons

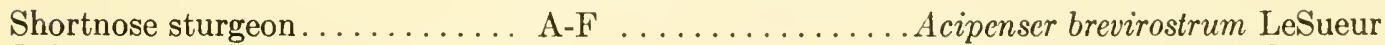

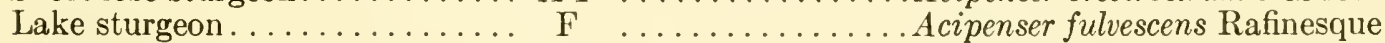

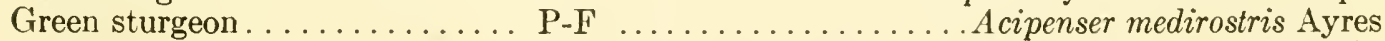
Atlantic sturgeon.......... A-F .............. icipenser oxyrhynchus Mitchill

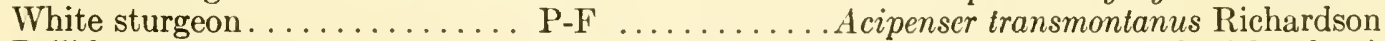
Pallid sturgeon............ F ....Scaphirhynchus albus (Forbes and Richardson) Shovelnose sturgeon......... F F .......Scaphirhynchus platorynchus (Rafinesque)

Polyodontidae-paddlefishes

Paddlefish. 


\section{Order Semionotiformes (Protospondyli and Ginglymodi)}

\section{Lepisosteidae-gars}

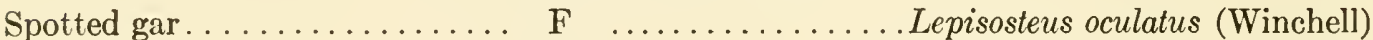

Longnose gar............. F F .............Lepisosteus osseus (Linnaeus)

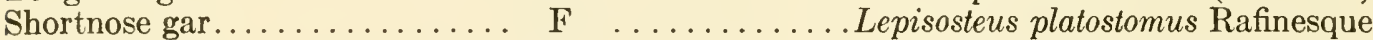

Florida gar.............. F ............Lepisosteus platyrhincus DeKay

Alligator gar............. F ...............episosteus spatula Lacépède

\section{Order Amifformes (Halecomorphi)}

Amiidae-bowfins

Bowfin................ F Amia calva Linnaeus

\section{Order Clupeiformes (Isospondy l)}

Elopidae-tarpons

Machete................. P-F

Ladyfish. . . . . . . . . . . . . A

Elops affinis Regan

Tarpon.

Albulidae-bonefishes

Bonefish

Blueback herring.......... A-F

Alabama shad ............... A-F

Skipjack herring. . . . . . . . . A-F

Hickory shad............. A

Ohio shad............... F

Alewife................ A-F

American shad ...............A-F-P

Finescale menhaden.......... A

Largescale menhaden.......... A

Yellowfin shad............. A

Atlantic menhaden........... A

Atlantic herring............ A

Pacific herring . . . . . . . . . . P

Gizzard shad .............. A-F

Threadfin shad ............ A-F

California round herring ....... P

Atlantic round herring........ A

False pilchard ............. A

Redear sardine............. A

Scaled sardine............ A

Flatiron herring............ P

Dwarf herring............ A

Pacific thread herring......... P

Atlantic thread herring. ....... A

Spanish sardine........... A

Pacific sardine ............... P
Alosa aestivalis (Mitchill) Alosa alabamae Jordan and Evermann . Alosa chrysochloris (Rafinesque) Alosa mediocris (Mitchill) . Alosa ohiensis Evermann Alosa pseudoharengus (Wilson) . Alosa sapidissima (Wilson) Brevoortia gunteri Hildebrand . Brevoortia patronus Goode Brevoortia smithi Hildebrand Brevoortia tyrannus (Latrobe) Clupea harengus harengus Linnaeus Clupea harengus pallasi Valenciemnes Dorosoma cepedianum (LeSueur) . . Dorosoma petenense (Günther)

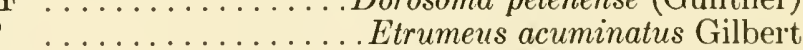
A..............Etrumeus sadina (Mitchill)

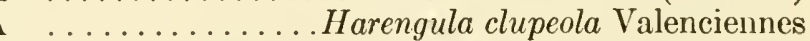
A ................ Harengula humeralis Cuvier A........Harengula pensacolae Goode and Bean P .........Harengula thrissina (Jordan and Gilbert) A .............. Jenkinsia lamprotaenia (Gosse) P ............ Opisthonema libertate (Günther)

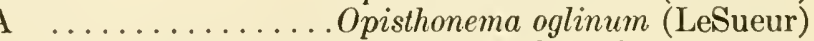

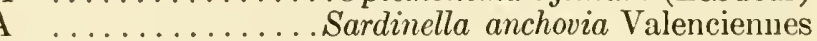

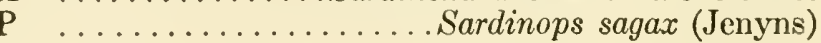


Engraulidae - anchovies

Key anchovy...............

Deepbody anchovy...........

Cuban anchovy.............

Slough anchovy............

New Jersey anchovy...........

Striped anchovy ............

Longnose anchovy............

Dusky anchovy.............

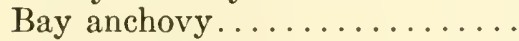

Silver anchovy.............

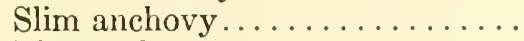

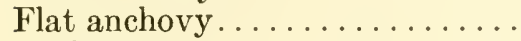

Anchoveta.

Northern anchovy.
A

$\mathrm{P}$

A

$\mathrm{P}$

A

A

A

A

A

A

$\mathrm{P}$

A

$\mathrm{P}$

$\mathrm{P}$
Anchoa cayorum (Fowler)

Anchoa compressa (Girard) Anchoa cubana (Poey)

Anchoa delicatissima (Girard)

. Anchoa duodecim (Cope)

Anchoa hepsetus (Linnaeus)

Anchoa lamprotaenia Hildebrand

Anchoa lyolepis (Evermann and Marsh)

Anchoa mitchilli (Valenciennes)

Anchoviella eurystole (Swain and Meek)

Anchoviella miarcha (Jordan and Gilbert)

. Anchoviella perfasciata (Poey)

Cetengraulis mysticetus (Günther)

Engraulis mordax Girard

Salmonidae - trouts, whitefishes, and graylings

Longjaw cisco............ F

Cisco or lake herring......... F

Arctic cisco............... F

Lake whitefish............ A-F

Bloater................. F

Deepwater cisco........... F

Kiyi................. F F

Broad whitefish............ F

Blackfin cisco............. F

Nipigon cisco............ F

Humpback whitefish......... F F

Shortnose cisco ............ F F

Least cisco............. F

Shortjaw cisco............ F

Pink salmon............. A-F-P

Chum salmon............. P-F

Coho salmon. ..............A-F-P

Sockeye salmon ${ }^{2} \ldots \ldots \ldots \ldots \ldots$. . P-F

Chinook salmon............. P-F

Bear Lake whitefish.......... F

Pygmy whitefish........... F

Round whitefish .......... F

Bonneville cisco............. F

Bonneville whitefish......... F

Mountain whitefish......... F

Golden trout............. F

Cutthroat trout........... P-F

Rainbow trout ${ }^{3} \ldots \ldots \ldots \ldots \ldots$ A-F-P.

Gila trout.............. F F

Atlantic salmon ${ }^{4}$.
Coregonus alpenae (Koelz)

Coregonus artedii LeSueur

Coregonus autumnalis (Pallas)

Coregonus clupeaformis (Mitchill)

Coregonus hoyi (Gill)

Coregonus johannae (Wagner)

Coregonus kiyi (Koelz)

Coregonus nasus (Pallas)

Coregonus nigripinnis (Gill)

Coregonus nipigon (Koelz)

Coregonus pidschian (Gmelin)

.Coregonus reighardi (Koelz)

Coregonus sardinella Valenciennes

Coregonus zenithicus (Jordan and Evermann)

Oncorhynchus gorbuscha (Walbaum)

Oncorhynchus keta (Walbaum)

Oncorhynchus kisutch (Walbaum)

. Oncorhynchus nerka (Walbaum)

Oncorhynchus tshawytscha (Walbaum)

Prosopium abyssicola (Snyder)

. Prosopium coulteri (Eigenmamn and Eigenmann)

Prosopium cylindraceum (Pallas)

Prosopium gemmiferum (Snyder)

Prosopium spilonotus (Snyder)

Prosopium williamsoni (Girard)

Salmo aguabonita Jordan

Salmo clarki Richardson

. Salmo gairdneri Richardson

Salmo gilae Miller

Salmo salar Linnaeus

\footnotetext{
1 The genus Coregonus is here employed to include species referred by some authors to Leucichthys.

2 Lacustrine sockeye salmon stocks are known as kokanee.

\& Rainbow trout are commonly called steelhead trout when they enter or return from the sea and large inland lakes. salmon.

Lake populations of Atlantic salmon are variously known as ouananiche, lake Atlantic salmon, landlocked salmon, and Sebago
} 
Brown trout............ A-F

Arctic char ${ }^{1}$.

Brook trout.

Dolly Varden.............. P-F

Lake trout............. F F

Inconnu.............. F

Arctic grayling........... F

Whitebait smelt............ P

Pond smelt............. P-F

Surf smelt.............. P-F

Capelin................ A-P

Arctic smelt............. P-F

American smelt........... A-F

Longfin smelt............ P-F

Night smelt............. P

Sacramento smelt........... P-F

Eulachon............. P-F $\mathrm{P}-\mathrm{F}$
$\mathrm{P}-\mathrm{F}$$\ldots \ldots \ldots \ldots \ldots$. . . Spirinchus thaleichthys (Ayres)

Salmo trutta Linnaeus Salvelinus alpinus (Linnaeus) Salvelinus fontinalis (Mitchill) . Salvelinus malma (Walbaum) . . . . . . . . Salvelinus namaycush (Walbaum) ..............Stenodus leucichthys (Güldenstadt) Osmeridae-smelts

Argentinidae-argentines

Pacific argentine........... P

Atlantic argentine.......... A . Allosmerus elongatus (Ayres) Hypomesus olidus (Pallas) Hypomesus pretiosus (Girard) ... Mallotus villosus (Müller) . Osmerus dentex Steindachner Osmerus morda.x (Mitchill) ......... Spirinchus dilatus Schultz and Chapman

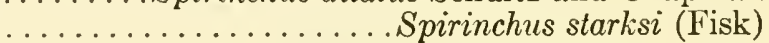

$\mathrm{P} \quad \ldots \ldots \ldots \ldots \ldots \ldots$. Argentina sialis Gilbert A $\quad \ldots \ldots \ldots \ldots \ldots \ldots$ Argentina silus Ascanius

Bathylagidae ${ }^{2}$-deepsea smelts

California smoothtongue....... P

Leuroglossus stilbius Gilbert

Opisthoproctidae ${ }^{3}$-barreleyes

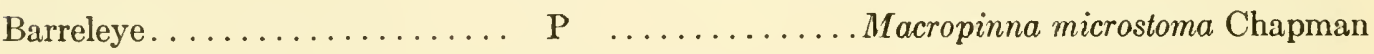

Hiodontidae-mooneyes

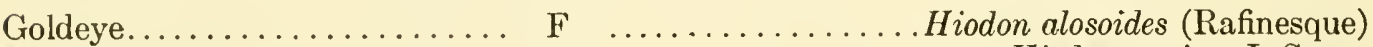

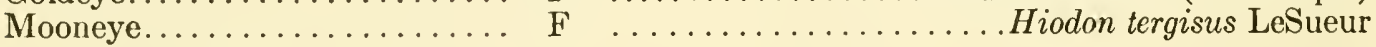

Umbridae-mudminnows

Alaska blackfish............ F

Olympic mudminnow........ F

Central mudminnow......... F

Eastern mudminnow........ F

Dallia pecloralis Bean Novumbra hubbsi Schultz Umbra limi (Kirtland) Umbra pygmaea (DeKay)

Esocidae-pikes

Redfin pickerel

F ..........Esox americanus americanus Gmelin Grass pickerel.

Esox americanus vermiculatus LeSueur

Chain pickerel Esox niger LeSueur

Northern pike.

$\mathrm{F}$

$\mathrm{F}$

Muskellunge ${ }^{4}$.

Esox lucius Linnaeus

$\mathrm{F}$ Esox masquinongy Mitchill

\footnotetext{
1 Sunape trout, blueback trout, and Quebec red trout are regarded by aome authors as species distinct from the Arctic char

2 Most deepsea amelts are bathypelagic; few enter water less than 100 fathoms deep.

Bathypelagic fishes of which one species hus been recorded as a straggler in our area.

- Spelled maskinonge in Ontario and Quebec.
} 


\section{Order Myctophiformes (Iniomi)}

\section{Synodontidae-lizardfishes}

Largescale lizardfish.......... . A

Smallscale lizardfish ........... A A

Shortjaw lizardfish.......... A

Inshore lizardfish........... A

Sand diver.............. A

California lizardfish ......... P

Offshore lizardfish............ A

Red lizardfish............. A

Snakefish . . . . . . . . . . . A
Saurida brasiliensis Norman Saurida caribbaea Breder Saurida normani Longley . Synodus foetens (Linnaeus) Synodus intermedius (Agassiz) Synodus lucioceps (Ayres) .Synodus poeyi Jordan Synodus synodus (Linnaeus) Trachinocephalus myops (Forster)

Scopelarchidae-pearleyes

Northern pearleye.

Myctophidae-lanternfishes

The many species of lanternfishes are chiefly bathypelagic, but occasional stragglers are found over the continental shelf.

Alepisauridae-lancetfishes

Longnose lancetfish A Alepisaurus ferox Lowe Pacific lancetfish............ P Alepisaurus richardsoni Bleeker

\section{Order Cypriniformes (Ostariophysi)}

Characidae-characins and tetras

Mexican tetra............ F .................... Ftyanax mexicanus (Filippi)

Cyprinidae-minnows and carps

Chiselmouth.............. F

Longfin dace............. F F

Stoneroller............... F F

Mexican stoneroller.......... F F

Goldfish. . ............... F

Northern redbelly dace....... F F

Southern redbelly dace........ F F

Finescale dace............. F F

Mountain redbelly dace....... F

Redside dace.............. F

Rosyside dace............ F F

Carp ................. F

Devils River minnow......... F

Roundnose minnow......... F

Ozark minnow............. F

Desert dace.............. F

Silverjaw minnow.......... F

Cutlips minnow............. F

Utah chub. ............... F

Blue chub............... F F

Thicktail chub............ F

Humpback chub............ F F
Acrocheilus alutaceus Agassiz and Pickering Agosia chrysogaster Girard

Campostoma anomalum (Rafinesque) .Campostoma ornatum Girard

Carassius auratus (Linnaeus) Chrosomus eos Cope Chrosomus erythrogaster (Rafinesque) Chrosomus neogaeus (Cope) Chrosomus oreas Cope Clinostomus elongatus (Kirtland) Clinostomus funduloides Girard .Cyprinus carpio Linnaeus Dionda diaboli Hubbs and Brown Dionda episcopa Girard . Dionda nubila (Forbes) ..........Eremichthys acros Hubbs and Miller ...Ericymba buccata Cope . . . . . . . . Exoglossum maxillingua (LeSueur) $\ldots \ldots \ldots \ldots \ldots \ldots \ldots$ Gila atraria (Girard) Gila bicolor (Girard) . . . . . . . Gila crassicauda (Baird and Girard)

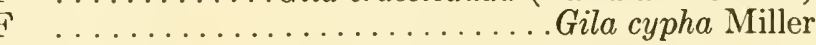


Sonora chub

Rio Grande chub............. F

Arroyo chub.............. F

Yaqui chub............ F

Bonytail............... F

Flame chub............. F

California roach .......... F

Brassy minnow ........... F

Cypress minnow........... F

Silvery minnow........... F

Speckled chub............ F

Bigeye chub............. F

Alabama chub............. F

Hornyhead chub........... F

Slender chub............ F

Oregon chub............. F

Streamline chub........... F

Sturgeon chub............ F

Flathead chub............ F

Redeye chub............ F

Highback chub. ........... F

Blotched chub............. F

Thicklip chub........... F F

Bluehead chub............ F

Sicklefin chub............. F

River chub............. F

Spotfin chub............ F

Lake chub.............. F

Rosyface chub........... F

Silver chub............ F

Gravel chub............. F

Least chub............... F

Hitch.................. F

Little Colorado spinedace...... F

Spikedace................. F

Moapa dace............ F

Peamouth............... F

Hardhead............... F

Golden shiner............. F

White shiner............ F

Whitemouth shiner......... F

Highfin shiner. . . . . . . . . . F

Texas shiner.............. F

Pallid shiner............. F

Comely shiner............ F

Satinfin shiner........... F

Pugnose shiner............. F

Rosefin shiner............ F

Popeye shiner........... F

Burrhead shiner........... $\mathrm{F}$

Emerald shiner........... F

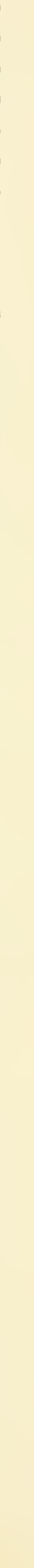

...................... Gila ditaenia Miller . Gila nigrescens (Girard) Gila orcutti (Eigenmann and Eigenmann) .Gila purpurea (Girard) Gila robusta Baird and Girard Hemitremia flammea (Jordan and Gilbert) Hesperoleucus symmetricus (Baird and Girard) Hybognathus hankinsoni Hubbs Hybognathus hayi Jordan Hybognathus nuchalis Agassiz .Hybopsis ${ }^{1}$ aestivalis (Girard) Hybopsis amblops (Rafinesque) Hybopsis bellica (Girard)

Hybopsis biguttata (Kirtland) Hybopsis cahni Hubbs and Crowe Hybopsis crameri Snyder

Hybopsis dissimilis (Kirtland) Hybopsis gelida (Girard) Hybopsis gracilis (Richardson) Hybopsis harperi (Fowler)

.Hybopsis hypsinotus (Cope) Hybopsis insignis Hubbs and Crowe .Hybopsis labrosa (Cope)

Hybopsis leptocephala (Girard) Hybopsis meeki Jordan and Evermann Hybopsis micropogon (Cope) Hybopsis monacha (Cope) Hybopsis plumbea (Agassiz) Hybopsis rubrifrons (Jordan) Hybopsis storeriana (Kirtland) Hybopsis x-punctata Hubbs and Crowe Iotichthys phlegethontis (Cope) .Lavinia exilicauda Baird and Girard . Lepidomeda vittata Cope ........................... Meda fulgida Girard Moapa coriacea Hubbs and Miller Mylocheilus caurinus (Richardson) Mylopharodon conocephalus (Baird and Girard) Notemigonus crysoleucas (Mitchill) .Notropis albeolus Jordan . Notropis alborus Hubbs and Raney Notropis altipinnis (Cope) Notropis amabilis (Girard) Notropis amnis Hubbs and Greene .Notropis amoenus (Abbott) Notropis analostanus (Girard) .Notropis anogenus Forbes .Notropis ardens (Cope) Notropis asperifrons Suttkus and Raney .Notropis atherinoides Rafinesque

\footnotetext{
'The genus IIybopsis is here employed to include species referred by some authors to Couesius, Erimystax, Extrarius, Macrhybopsis, Nocomis, Oregonichthys, and Platygobio.
} 
Blackspot shiner........... F

Rough shiner............. F

Red River shiner............ F

Pretty shiner............ F

Bridle shiner............ F

River shiner.............. F

Bigeye shiner............ F

Tamaulipas shiner.......... F

Brazos shiner............ F

Smalleye shiner............ F

Ghost shiner............. F

Blue shiner............... F F

Oemulgee shiner........... F

Alabama shiner........... F F

Bluestripe shiner.......... F

Bluntface shiner........... F

Crescent shiner............ F

Ironeolor shiner............. F

Chihuahua shiner........... F

Redlip shiner............ F

Greenfin shiner............. F

Greenhead shiner........... F

Rainbow shiner........... F

Warpaint shiner............ F

Common shiner........... F

Dusky shiner............. F

Bigmouth shiner........... F

Broadstripe shiner.......... F

Ribbon shiner............ F

Whitetail shiner............ F

Arkansas River shiner........ F F

Wedgespot shiner........... F

Blackehin shiner........... F

Blacknose shiner........... F

Spottail shiner............ F

Sailfin shiner............ F

Highscale shiner. . . . . . . . . F F

Silverband shiner.......... F

Rio Grande shiner........... F F

Ohoopee shiner............ F

Plateau shiner.............. F F

Tennessee shiner............ F

Mountain shiner.......... F

Longnose shiner............ F

Yellowfin shiner............. F

Red shiner.............. F

Taillight shiner........... F

Pinewoods shiner............ F

Whitefin shiner........... F

Kiamichi shiner............ F

Sharpnose shiner........... F

Ozark shiner.............. F

Plains shiner............ F

Colorless shiner........... F
.Notropis atrocaudalis Evermann Notropis baileyi Suttkus and Raney Notropis bairdi Hubbs and Ortenburger .Notropis bellus (Hay) Notropis bifrenatus (Cope) Notropis blennius (Girard) Notropis boops Gilbert Notropis braytoni Jordan and Evermann Notropis brazosensis Hubbs and Bonham Notropis buccula Cross Notropis buchanani Meek Notropis caeruleus (Jordan) Notropis callisema (Jordan) . Notropis callistius (Jordan) Notropis callitaenia Bailey and Gibbs Notropis camurus (Jordan and Meek) Notropis cerasinus (Cope) . Notropis chalybaeus (Cope) . Notropis chihuahua Woolman Notropis chiliticus (Cope)

Notropis chloristius (Jordan and Brayton) . Notropis chlorocephalus (Cope)

. Notropis chrosomus (Jordan) Notropis coccogenis (Cope) Notropis cornutus (Mitchill) Notropis cummingsae Myers Notropis dorsalis (Agassiz) Notropis euryzonus Suttkus Notropis fumeus Evermann . Notropis galacturus (Cope) Notropis girardi Hubbs and Ortenburger Notropis greenei Hubbs and Ortenburger .Notropis heterodon (Cope)

Notropis heterolepis Eigenmann and Eigenmann Notropis hudsonius (Clinton) Notropis hypselopterus (Günther) Notropis hypsilepis Suttkus and Raney Notropis illecebrosus (Girard) . Notropis jemezanus (Cope) .Notropis leedsi Fowler .Notropis lepidus (Girard) Notropis leuciodus (Cope) .Notropis lirus (Jordan) Notropis longirostris (Hay)

Notropis lutipinnis (Jordan and Brayton) .Notropis lutrensis (Baird and Girard) . Notropis maculatus (Hay) Notropis matutinus (Cope) .Notropis niveus (Cope) Notropis ortenburgeri Hubbs ...................... Notropis oxyrhynchus Hubbs and Bonham .Notropis ozarcanus Meek Notropis percobromus (Cope) Notropis perpallidus Hubbs and Black 
Coastal shiner........... F

Silver shiner............. F

Chub shiner.............. F

Swallowtail shiner.......... F

Proserpine shiner.......... F

Fieryblack shiner........... F

Cherryfin shiner........... F

Rosyface shiner............ F

Saffron shiner............ F

Sabine shiner........... F

New River shiner........... F

Sandbar shiner............ F

Flagfin shiner............. F

Bluntnose shiner.......... F

Mirror shiner............. F

Spotfin shiner........... F

Silverstripe shiner.......... F

Sand shiner............. F

Weed shiner.............. F

Topeka shiner............ F F

Tricolor shiner........... F

Redfin shiner............. F F

Stargazing shiner........... F

Blacktail shiner........... F

Mimic shiner............. F F

Bluenose shiner........... F

Steelcolor shiner........... F

Coosa shiner.............. F

Altamaha shiner........... F

Bleeding shiner . . . . . . . . . F

Bandfin shiner............ F

Pugnose minnow........... F F

Sacramento blackfish......... F F

Tonguetied minnow.......... F

Riffle minnow............ F

Suckermouth minnow........ F

Kanawha minnow........... F

Stargazing minnow........... F

Bluntnose minnow.......... F

Fathead minnow........... F

Slim minnow............ F

Bullhead minnow.......... F

Woundfin............. F

Splittail.............. F

Sacramento squawfish........ F

Colorado squawfish.......... F F

Northern squawfish. . . . . . . . . F

Umpqua squawfish........... F

Blacknose dace........... F F

Longnose dace. . . . . . . . . . . F F

Umpqua dace............. F F

Leopard dace............. F

Speckled dace............ F

Bitterling .............. F
.Notropis petersoni Fowler

Notropis photogenis (Cope)

.Notropis potteri Hubbs and Bonham

Notropis procne (Cope)

Notropis proserpinus (Girard)

.Notropis pyrrhomelas (Cope)

. Notropis roseipinnis (Hay)

. Notropis rubellus (Agassiz)

Notropis rubricroceus (Cope)

. Notropis sabinae Jordan and Gilbert

Notropis scabriceps (Cope)

Notropis scepticus (Jordan and Gilbert)

Notropis signipinnis Bailey and Suttkus

.Notropis simus (Cope)

Notropis spectrunculus (Cope)

.Notropis spilopterus (Cope)

.Notropis stilbius (Jordan)

Notropis stramineus (Cope)

Notropis texanus (Girard)

.Notropis topeka Gilbert

Notropis trichroistius (Jordan and Gilbert)

Notropis umbratilis (Girard)

Notropis uranoscopus Suttkus

Notropis venustus (Girard)

Notropis volucellus (Cope)

. Notropis welaka Evermann and Kendall

Notropis whipplei (Girard)

Notropis xaenocephalus (Jordan)

Notropis xaenurus (Jordan)

Notropis zonatus (Agassiz)

Notropis zonistius (Jordan)

Opsopoeodus emiliae Hay

Orthodon microlepidotus (Ayres)

Parexoglossum laurae Hubbs

. Phenacobius catostomus Jordan

Phenacobius mirabilis (Girard)

................. Phenacobius teretulus Cope

Phenacobius uranops Cope

Pimephales notatus (Rafinesque)

Pimephales promelas Rafinesque

.Pimephales tenellus (Girard)

Pimephales vigilax (Baird and Girard)

Plagopterus argentissimus Cope

Pogonichthys macrolepidotus (Ayres)

Ptychocheilus grandis (Ayres)

Ptychocheilus lucius Girard

.Ptychocheilus oregonensis (Richardson)

Ptychocheilus umpquae Snyder

Rhinichthys atratulus (Hermann)

Rhinichthys cataractae (Valenciennes)

Rhinichthys evermanni Snyder

Rhinichthys falcatus (Eigenmann and Eigenmann)

Rhinichthys osculus (Girard)

Rhodeus sericeus (Pallas) 
Redside shiner............ F F

Lahontan redside............ F

Rudd................... F

Creek chub ............... F F

Fallfish.............. F

Pearl dace............... F

Tui chub............... F

Mohave chub............. F

Leatherside chub.......... F F

Loach minnow............. F F

Tench................ F
Richardsonius balteatus (Richardson)

Richardsonius egregius (Girard)

Scardinius erythrophthalmus (Linnaeus)

Semotilus atromaculatus (Mitchill)

Semotilus corporalis (Mitchill)

Semotilus margarita (Cope)

Siphateles bicolor (Girard)

.............. Siphateles mohavensis Snyder

........ Snyderichthys copei (Jordan and Gilbert)

Tiaroga cobitis Girard

Tinca tinca (Linnaeus)

Catostomidae-suckers

River carpsucker

Quillback.

Plains carpsucker

Highfin carpsucker

Utah sucker

Longnose sucker

Bridgelip sucker

White sucker.

Webug sucker.

Sonora sucker

Flannelmouth sucker. . . . . . . . . . .

Lost River sucker.

Largescale sucker .

Modoc sucker.

Sacramento sucker.

Klamath smallscale sucker

Klamath largescale sucker.

Tahoe sucker.

Warner sucker.

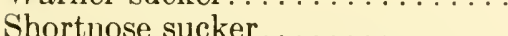

Cui-ui ${ }^{1} \ldots \ldots \ldots \ldots \ldots \ldots \ldots \ldots$

June sucker . . . . . . . . . . . . .

Blue sucker. . . . . . . . . . . . .

Creek chubsucker............ F

Lake chubsucker............. F F

Sharpfin chubsucker.......... F

Alabama hog sucker......... F F

Northern hog sucker.......... F F

Roanoke hog sucker.......... F

Smallmouth buffalo........... F F

Bigmouth buffalo........... F F

Black buffalo.............. F

Harelip sucker. . . . . . . . . . . F F

Spotted sucker............ F

Silver redhorse.............. F

Bigeye jumprock............ F F

Blackfin sucker.
$\mathrm{F}$

$\mathrm{F}$

F

$\mathrm{F}$

$\mathrm{F}$

F

F $\mathrm{F}$ $\mathrm{F}$ F F $\mathrm{F}$

$$
\text { . }
$$$$
\text { ...... Hypentelium roanokense Raney and Lachner }
$$$$
\text { ................. Ictiobus bubalus (Rafinesque) }
$$$$
\text { . . . . . . . Ictiobus cyprinellus (Valenciennes) }
$$$$
\text { . . . . . . . . . . Ictiobus niger (Rafinesque) }
$$$$
\text { . . . . . . . Lagochila lacera Jordan and Brayton }
$$$$
\text { .............Minytrema melanops (Rafinesque) }
$$$$
\text { . Moxostoma anisurum (Rafinesque) }
$$

Moxostoma ariommum Robins and Raney .Moxostoma atripinne Bailey

\footnotetext{
1 Pronounced kweé-wee.
} 
Shorthead redhorse.......... F

River redhorse.............. F

Black jumprock............. F F

V-lip redhorse........... F

Gray redhorse............ F

Carolina redhorse........... F

Black redhorse.............. F

Golden redhorse............. F

Rustyside sucker............ F F

Copper redhorse........... F

Greater jumprock........... F

Neuse redhorse............ F

Northern redhorse............ F

Suckermouth redhorse........ F F

Blacktail redhorse............ F

Torrent sucker............. F

Smallfin redhorse........... F

Striped jumprock ........... F

Greater redhorse.......... F

Gila sucker.............. F

Bluehead sucker............ F

White River sucker........... F

Mountain sucker........... F

Rio Grande sucker. . . . . . . . . . F F

Lahontan sucker............ F F

Santa Ana sucker........... F F

Green sucker............... F

Humpback sucker............ F

\section{Cobitidae-loaches}

F
Moxostoma breviceps (Cope)

Moxostoma carinatum (Cope)

Moxostoma cervinum (Cope)

.Moxostoma collapsum (Cope)

Moxostoma congestum (Baird and Girard)

Moxostoma coregonus (Cope)

Moxostoma duquesnei (LeSueur)

Moxostoma erythrurum (Rafinesque)

Moxostoma hamiltoni (Raney and Lachner)

. Moxostoma hubbsi Legendre

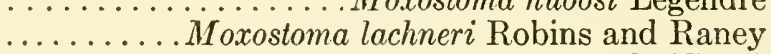

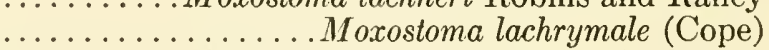
. Moxostoma macrolepidotum (LeSueur)

Moxostoma pappillosum (Cope)

Moxostoma poecilurum (Jordan)

Moxostoma rhothoecum (Thoburn) Moxostoma robustum (Cope)

Moxostoma rupiscartes Jordan and Jenkins

Moxostoma valenciennesi Jordan

Pantosteus clarki (Baird and Girard)

Pantosteus delphinus (Cope)

Pantosteus intermedius (Tanner)

Pantosteus platyrhynchus (Cope)

Pantosteus plebeius (Baird and Girard)

Pantosteus lahontan Rutter

Pantosteus santaanae Snyder

Pantosteus virescens Cope

Xyrauchen texanus (Abbott)

Misgurnus anguillicaudatus (Cantor)

Ariidae-sea catfishes

A

Bagre marinus (Mitchill)

Gafftopsail catfish

A

Galeichthys felis (Linnaeus)

\section{Ictaluridae-freshwater catfishes}

White catfish

Blue catfish

Headwater catfish

Black bullhead.

Yellow bullhead.

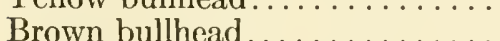

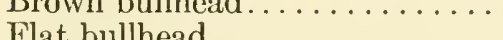

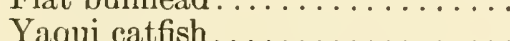

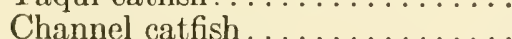

Mountain madtom............

Slender madtom. ........... I

Stonecat............... F

Black madtom............ F

Carolina madtom ........... F

Orangefin madtom .......... F

Tadpole madtom.
F

F

F

F

F

$\mathrm{F}$

F

F

$\mathrm{F}$

$\mathrm{F}$ F ( F
Ictalurus catus (Linnaeus)

Ictalurus furcatus (LeSueur) Ictalurus lupus (Girard)

Ictalurus melas (Rafinesque)

Ictalurus natalis (LeSueur)

Ictalurus nebulosus (LeSueur) Ictalurus platycephalus (Girard) Ictalurus pricei (Rutter) Ictalurus punctatus (Rafinesque) . Noturus eleutherus Jordan .Noturus exilis Nelson

Noturus flavus Rafinesque Noturus funebris Gilbert and Swain . Noturus furiosus Jordan and Meek Noturus gilberti Jordan and Evermann Noturus gyrinus (Mitchill) 
Least madtom............. F

Margined madtom......... F

Speckled madtom ........... F

Brindled madtom........... F

Freckled madtom ........... F

Flathead catfish............ F

Widemouth blindcat......... F F

Toothless blindeat.......... F
. Noturus hildebrandi (Bailey and Taylor) . Noturus insignis (Richardson) . Noturus leptacanthus Jordan . Noturus miurus Jordan Noturus nocturnus Jordan and Gilbert Pylodictis olivaris (Rafinesque) Satan eurystomus Hubbs and Bailey Trogloglanis pattersoni Eigenmann

\section{Order Anguilliformes (Apodes)}

Anguillidae-freshwater eels

American eel . A-F Anguilla rostrata (LeSueur)

Muraenidae-morays

Pygmy moray

A A

Viper moray.............. A

Green moray ............. A

California moray........... P

Spotted moray............ A

Blackedge moray............ A

Purplemonth moray.......... A

Goldentail moray............ A

Reticulate moray........... A Anarchias yoshiae Kanazawa Echidna catenata (Bloch) Enchelycore nigricans (Bonnaterre) Gymnothorax funebris Ranzani Gymnothorax mordax (Ayres) Gymnothorax moringa (Cuvier)

Nemichthyidae-snipe eels

Fishes of this family inhabit the deep seas and thus are not members of the fauna covered in this list.

\section{Congridae-conger eels}

Bandtooth conger.......... A

Margintail conger........... A

Conger eel.............. A

Manytooth conger........... A

Yellow conger.............. A

Whiptail conger............ A

Silver conger............... A

Slender pike eel............ A

Catalina conger........... P
Ariosoma impressa (Poey) Conger caudilimbatus (Poey)

Conger oceanicus (Mitchill)

Conger triporiceps Kanazawa Congrina flava (Goode and Bean)

Congrina gracilior Ginsburg Hoplunnis macrurus Ginsburg Neoconger mucronatus Girard . Rhynchocymba catalinensis Wade

\section{Ophichthidae-snake eels}

Key worm eel............. A

Whip eel............... A

Sooty eel................ A

Surf eel................ A

Blotched snake eel.......... A

Shorttail snake eel........... A

Ridgefin eel............... A

Horsehair eel............ A

Thread eel................ A

California worm eel.......... P

Sailfin eel................ A

Sharptail eel.............. A
Ahlia egmontis (Jordan) Bascanichthys scuticaris (Goode and Bean) . Bascanichthys teres (Goode and Bean) . Caecula conklini (Eigenmann) Callechelys muraena Jordan and Evermann Callechelys perryae Storey Cryptopterygium holochroma Ginsburg Gordiichthys irretitus Jordan and Davis .................... Gordiichthys springeri Ginsburg ........... Hesperomyrus fryi Myers and Storey ............ Letharchus velifer Goode and Bean

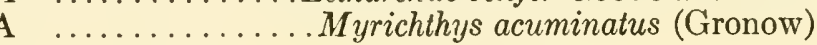


Goldspotted eel..............

Tiger snake eel.

Speckled worm eel

Spotted spoon-nose eel

Snapper eel................

Stippled spoon-nose eel.........

Shrimp eel

Palespotted eel.

Spotted snake eel

Pacific snake eel.

Yellow snake eel.

Finless eel.
A

$\mathrm{P}$

A

A

A

A

A

A

A

$\mathrm{P}$

$\mathrm{P}$

A
Myrichthys oculatus (Kaup)

. Myrichthys tigrinus Girard

.Myrophis punctatus Lütken Mystriophis intertinctus (Richardson) .Mystriophis mordax (Poey)

Mystriophis punctifer (Kaup)

Ophichthus gomesi (Castelnau)

.Ophichthus ocellatus (LeSueur)

.Ophichthus ophis (Linnaeus)

Ophichthus triserialis (Kaup)

Ophichthus zophochir (Jordan and Gilbert) Verma kendalli (Gilbert)

Dysommidae - mustard eels

Mustard eel

A

Dysomma aphododera Ginsburg

\section{Order Notacanthiformes (Heteromi)}

Notacanthidae-spiny eels

Spiny eel.

$\mathrm{A}$

.Notacanthus nasus Bloch

\section{Order Beloniformes (Synentognathi)}

Scomberesocidae-sauries

Pacific saury.............. P

Atlantic saury............ A
Cololabis saira (Brevoort)

.Scomberesox saurus (Walbaum)

Belonidae-needlefishes

Flat needlefish

Agujon

California needlefish

Atlantic needlefish

Redfin needlefish.

Houndfish

Timucu.
A

A

$\mathrm{P}$

$\mathrm{A}$

A

A

A
-Ablennes hians (Valenciennes)

. . Strongylura acus (Lacépède)

Strongylura exilis (Girard)

Strongylura marina (Walbaum)

......Strongylura notata (Poey)

Strongylura raphidoma (Ranzani)

. Strongylura timucu (Walbaum)

\section{Hemiramphidae - halfbeaks}

Hardhead halfbeak........... A

Flying halfbeak............. A

Ribbon halfbeak............ P

Balao.................. A

Ballyhoo.

Longfin halfbeak.

California halfbeak.......... P

Halfbeak.
A

Chriodorus atherinoides Goode and Bean ................Euleptorhamphus velox Poey ........Euleptorhamphus viridis (van Hasselt)

.................. Hemiramphus balao LeSueur

A $\quad . . \ldots \ldots . . . . H e m i r a m p h u s$ brasiliensis (Linnaeus)

P .......Hemiramphus saltator Gilbert and Starks

P .......Hyporhamphus rosae (Jordan and Gilbert)

A-P .........Hyporhamphus unifasciatus (Ranzani)

Exocoetidae-flyingfishes

California flyingfish

Clearwing flyingfish

Margined flyingfish

Bandwing flyingfish

Spotfin flyingfish.

Atlantic flyingfish
$\mathbf{P}$

A

A

A

A

A
Cypselurus californicus (Cooper)

Cypselurus comatus (Mitchill)

Cypselurus cyanopterus (Valenciennes)

Cypselurus exsiliens (Linnaeus)

.Cypselurus furcatus (Mitchill)

Cypselurus heterurus (Rafinesque) 
Oceanic two-wing flyingfish..... A A

Tropical two-wing flyingfish ..... A

Sharpchin flyingfish......... P

Fourwing flyingfish .......... A

Smallwing flyingfish ......... A

Sailfin flyingfish............. A

Bluntnose flyingfish.......... A

Blackwing flyingfish.......... P
A

A

A

A

A

$\mathrm{P}$

\section{Order Cyprinodontiformes (Microcyprini)}

Cyprinodontidae-killifishes

Diamond killifish............. A

White River killifish.......... F F

Railroad Valley killifish........ F

Leon Springs pupfish.......... F F

Devils Hole pupfish........... F F

Comanche Springs pupfish..... . F

Lake Eustis minnow........... F F

Desert pupfish............ F

Nevada pupfish............ F F

Owens River pupfish......... F F

Red River pupfish............ F

Salt Creek pupfish........... F

Sheepshead minnow.......... A-F

Pahrump killifish........... F F

Ash Meadows killifish.......... F F

Goldspotted killifish.......... A

Whiteline topminnow......... F

Northern studfish............ F

Golden topminnow.......... F

Banded topminnow.......... F F

Marsh killifish............ A-F

Banded killifish............ F F

Gulf killifish............... A

Mummichog.............. A-F

Saltmarsh topminnow......... A-F

Plains killifish............. F F

Spotfin killifish............. A

Striped killifish.............. A

Blackstripe topminnow........ F

Starhead topminnow.......... F

Blackspotted topminnow...... F F

California killifish............ P-F

Bayou killifish............ A-F

Speckled killifish............. F

Plains topminnow........... F

Seminole killifish............ F

Longnose killifish............ A

Southern studfish........... F

Waccamaw killifish........... F

Rio Grande killifish........... F F

Flagfish............... F
Exocoetus obtusirostris Günther Exocoetus volitans Linnaeus Fodiator acutus (Valenciennes) Hirundichthys affinis (Günther) Oxyporhamphus micropterus (Valenciennes) Parexocoetus brachypterus (Richardson) Prognichthys gibbifrons (Valenciennes) . Prognichthys rondeleti (Valenciennes)
............Adinia xenica (Jordan and Gilbert) ............... Crenichthys baileyi (Gilbert) Cyprinodon bovinus Baird and Girard Cyprinodon diabolis Wales Cyprinodon elegans Baird and Girard Cyprinodon hubbsi Carr Cyprinodon macularius Baird and Girard Cyprinodon nevadensis

Eigenmann and Eigenmann Cyprinodon radiosus Miller Cyprinodon rubrofluviatilis Fowler Cyprinodon salinus Miller Cyprinodon variegatus Lacépède .Empetrichthys latos Miller Empetrichthys merriami Gilbert .Floridichthys carpio (Günther) Fundulus albolineatus Gilbert Fundulus catenatus (Storer) Fundulus chrysotus (Günther) Fundulus cingulatus Valenciennes Fundulus confluentus Goode and Bean Fundulus diaphanus (LeSueur) Fundulus grandis Baird and Girard .Fundulus heteroclitus (Linnaeus) . Fundulus jenkinsi (Evermann) Fundulus kansae Garman Fundulus luciae (Baird) Fundulus majalis (Walbaum) Fundulus notatus (Rafinesque) Fundulus notti (Agassiz) Fundulus olivaceus (Storer) Fundulus parvipinnis Girard Fundulus pulvereus (Evermann) Fundulus rathbuni Jordan and Meek Fundulus sciadicus Cope Fundulus similis (Baird and Girard) Fundulus stellifer (Jordan)

Fundulus waccamensis Hubbs and Raney Fundulus zebrinus Jordan and Gilbert Jordanella floridae Goode and Bean 
Pygmy killifish............ F

Bluefin killifish............. $\mathrm{F}$

Rainwater killifish.......... A-F

Rivulus. .
Leptolucania ommata (Jordan) Lucania goodei Jordan
Lucania parva (Baird and Girard) .Rivulus marmoratus Poey

\section{Poeciliidae-livebearers}

Mosquitofish.............. A-F

Big Bend gambusia.......... F

Largespring gambusia......... F

Clear Creek gambusia.......... F

Pecos gambusia............ F

Blotched gambusia.......... F

Least killifish............ F

Amazon molly............ F

Sailfin molly............. A-F

Gila topminnow........... F
Gambusia affinis (Baird and Girard) Gambusia gaigei Hubbs Gambusia geiseri Hubbs and Hubbs Gambusia heterochir Hubbs Gambusia nobilis (Baird and Girard) Gambusia senilis Girard Heterandria formosa Agassiz Mollienesia formosa (Girard) Mollienesia latipinna LeSueur Poeciliopsis occidentalis (Baird and Girard)

Amblyopsidae - cavefishes

Ozark cavefish........... F

Northern cavefish.......... F

Spring cavefish........... F

Swampfish.............. F

Southern cavefish.
Amblyopsis rosae (Eigenmann) Amblyopsis spelaea DeKay Chologaster agassizi Putnam .Chologaster cornuta Agassiz Typhlichthys subterraneus Girard

\section{Order Gadiformes (Anacanthini)}

Gadidae - codfishes and hakes

Polar cod................ A

Arctic cod............... A-P

Cusk.................. A

Saffron cod............... P

Fourbeard rockling........... A

Pacific cod................. . P

Atlantic cod............... A

Greenland cod............ A

Burbot.................. F

Haddock.................. A

Silver hake................ A

Pacific hake................. P

Pacific tomeod............... P

Atlantic tomeod.............. A

Longfin hake............... A

Pollock.................... A

Walleye pollock............ P

Squirrel hake.............. A

Gulf hake............... A

Carolina hake............. A

Southern hake............ A

Spotted hake................ A

White hake............. A
Arctogadus glacialis (Peters)

Boreogadus saida (Lepechin) Brosme brosme (Müller) Eleginus gracilis (Tilesius) Enchelyopus cimbrius (Linnaeus) .Gadus macrocephalus Tilesius . Gadus morhua Linnaeus .Gadus ogac Richardson Lota lota (Linnaeus)

Melanogrammus aeglefinus (Linnaeus) . Merluccius bilinearis (Mitchill) .Merluccius productus (Ayres) Microgadus proximus (Girard) Microgadus tomcod (Walbaum) .Phycis chesteri Goode and Bean ... Pollachius virens (Linnaeus) Theragra chalcogrammus (Pallas) Urophycis chuss (Walbaum) Urophycis cirratus (Goode and Bean) . Urophycis earlli Bean ....... Urophycis floridanus (Bean and Dresel) ................. Urophycis regius (Walbaum) .. Urophycis tenuis (Mitchill)

\section{Macrouridae-grenadiers ${ }^{1}$}

Marlin-spike.

\footnotetext{
1 Most grenadiers, or rat-tails, live in deep water, but one species is found in Atlantic shore waters.
} 


\section{Order Gasterosteiformes (Thoracostei + Hemibranchi + Lophobranchi + SOLENICHTHYES)}

\section{Gasterosteidae-sticklebacks}

Fourspine stickleback........ A-F ............... peltes quadracus (Mitchill)

Brook stickleback.......... F .............. Eucalia inconstans (Kirtland)

Threespine stickleback........A-F-P . . . . . . . . Gasterosteus aculeatus Linnaeus

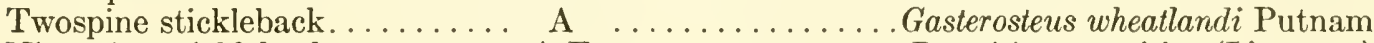

Ninespine stickleback....... A-F .......... Pungitius pungitius (Linnaeus)

\section{Aulorhynchidae - tube-snouts}

Tube-snout............ P . . . . . . . . . . A ulorhynchus flavidus Gill

Aulostomidae-trumpetfishes

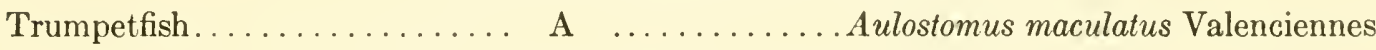

Fistulariidae-cornetfishes

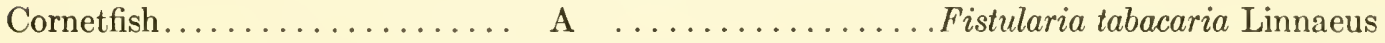

\section{Centriscidae - snipefishes}

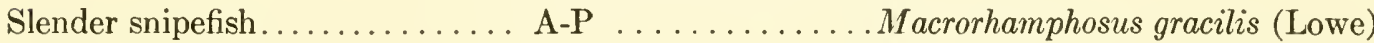

Longspine snipefish......... A .........Macrorhamphosus scolopax (Linnaeus)

Syngnathidae - pipefishes and seahorses

Whitenose pipefish.......... A

Crested pipefish........... A

Swivelhead pipefish.......... P

Spotted seahorse............ A

Pacific seahorse............. P

Offshore seahorse............ A

Dwarf seahorse............. A

Fringed pipefish............ A

Insular pipefish............ A

Banded pipefish........... A

Opossum pipefish........... A

Snubnose pipefish........... P

Barred pipefish............. P

Kelp pipefish ............... P

Pugnose pipefish........... A

Shortfin pipefish............ A

Dusky pipefish............ A

Northern pipefish........... A

Bay pipefish............. P

Chain pipefish........... A

Sargassum pipefish.......... A

Gulf pipefish............. A

Bull pipefish............ A
Corythoichthys albirostris Heckel Corythoichthys brachycephalus (Poey)

Dermatostethus punctipinnis Gill Hippocampus erectus Perry Hippocampus ingens Girard Hippocampus obtusus Ginsburg Hippocampus zosterae Jordan and Gilbert Micrognathus crinigerus (Bean and Dresel) Micrognathus crinitus (Jenyns) Micrognathus vittatus (Kaup) .. Oostethus lineatus (Kaup) .......Syngnathus arctus (Jenkins and Evermann) Syngnathus auliscus (Swain) ............ Syngnathus californiensis Storer ............. Syngnathus dunckeri Metzelaar ..................... Syngnathus elucens Poey Syngnathus floridae (Jordan and Gilbert) ............... . Syngnathus fuscus Storer Syngnathus griseolineatus Ayres . Syngnathus louisianae Günther Syngnathus pelagicus Linnaeus Syngnathus scovelli (Evermann and Kendall) . Syngnathus springeri Herald

\section{ORDER LAMPRIDIFORMes (Allotriognathi)}

Lamprididae-opahs

Opah 
Stylephoridae-tube-eyes

Tube-eye.

Lophotidae - crestfishes

Crestfish A-P .Lophotus capellei (Schlegel)

Regalecidae-oarfishes

Oarfish A-P Regalecus glesne (Ascanius)

Trachipteridae-ribbonfishes

Dealfish................. A Trachipterus arcticus (Brünnich)

Scalloped ribbonfish.......... A-P

Polka-dot ribbonfish.......... A-P

King-of-the-salmon . $\mathrm{P}$ $\ldots \ldots \ldots \ldots \ldots$ Trachipterus cristatus Bonelli ............ Trachipterus trachypterus (Gmelin)

\section{Order Beryciformes (Berycoidei)}

Polymyxiidae-beardfishes

Beardfish

A Polymixia lowei Günther

Holocentridae - squirrelfishes and soldierfishes

Squirrelfish

Deepwater squirrelfish.

Reef squirrelfish...............

Longjaw squirrelfish..........

Longspine squirrelfish..........

Dusky squirrelfish............

Blackbar soldierfish............

Bigeye soldierfish...............

Cardinal soldierfish.
A A A A A A A A A
Holocentrus ascensionis (Osbeck) Holocentrus bullisi Woods . Holocentrus coruscus (Poey) Holocentrus marianus (Cuvier) Holocentrus rufus (Walbaum) Holocentrus vexillarius (Poey) . Myripristis jacobus Cuvier Ostichthys trachypomus (Günther) Plectrypops retrospinis (Guichenot)

Order Percopsiformes (Salmopercae)

Percopsidae - trout-perches

Trout-perch.............. F . . ........ Percopsis omiscomaycus (Walbaum) Sand roller................ F .Percopsis transmontana

(Eigenmann and Eigenmann)

Aphredoderidae-pirate perches

Pirate perch............. F ............ Aphredoderus sayanus (Gilliams)

Order Zeiformes (Zeomorphi)

Zeidae-dories

American John Dory......... A Zenopsis ocellata (Storer)

Caproidae-boarfishes

Deepbody boarfish

A Antigonia capros Lowe

Shortspine boarfish A ...........Antigonia combatia Berry and Rathjen

Order Perciformes (Percomorphi; Acanthopterygit)

Centropomidae-snooks

Swordspine snook

A Centropomus ensiferus Poey

Little snook.

A Centropomus parallelus Poey 
Tarpon snook.

Snook.
$\mathrm{A}$

A
Centropomus pectinatus Poey Centropomus undecimalis (Bloch)

Serranidae ${ }^{1}$-sea basses

Mutton hamlet............. A

Crimson bass.............. A

Southern sea bass........... A

Bank sea bass.............. A

Rock sea bass............. A

Black sea bass............. A

Coney................. A

Marbled grouper............ A

Dwarf sand perch........... A

Sand perch.............. A

Rock hind .............. A

Spotted cabrilla............. P

Speckled hind............. A

Yellowedge grouper.......... A

Red hind................. A

Jewfish................ A

Red grouper.............. A

Warsaw grouper........... A

Snowy grouper............. A

Nassau grouper............. A

Spanish flag............. A

Longtail bass............. A

Splittail bass............. P

Red barbier................ A

Butter hamlet............. A

Black grouper............. A

Gulf grouper............. P

Gag................. A

Scamp................ A

Tiger grouper.

Yellowfin grouper

Broomtail grouper.

Roughtongue bass .

Blackear bass.

Kelp bass. ..........................

Spotted sand bass.

Sand bass.

Creole-fish.

Graysby

Yellowtail bass

Wreckfish

Orangeback bass

Lantern bass . .

Saddle bass.

Tattler.

Streamer bass

Reef bass.

White perch.

$\mathrm{P}$

A

A

$\mathrm{P}$

$\mathrm{P}$

$P$

A

A

A

A

A

A

A

A

A

A
A

A

........ Centropristes philadelphicus (Linnaeus)

................... Centropristes striatus (Linnaeus)

................. Cephalopholis fulva (Linnaeus)

.......... Dermatolepis inermis (Valenciennes)

.........Diplectrum bivittatum (Valenciennes)

............ Diplectrum formosum (Linnaeus)

............. Epinephelus adscensionis (Osbeck)

..................Epinephelus analogus Gill

Epinephelus drummondhayi Goode and Bean

Epinephelus flavolimbatus Poey

Epinephelus guttatus (Linnaeus)

Epinephelus itajara (Lichtenstein)

Epinephelus morio (Valenciennes)

Epinephelus nigritus (Holbrook)

Epinephelus niveatus (Valenciennes)

... Epinephelus striatus (Bloch)

Gonioplectrus hispanus (Cuvier)

Hemanthias leptus (Ginsburg)

Hemanthias peruanus (Steindachner)

Hemanthias vivanus (Jordan and Swain)

Hypoplectrus unicolor (Walbaum)

.................ycteroperca bonaci (Poey)

Mycteroperca jordani (Jenkins and Evermann) .Mycteroperca microlepis (Goode and Bean) Mycteroperca phenax Jordan and Swain

A ................ ycteroperca tigris (Valenciennes)

A ...................

A-F
. Mycteroperca xenarcha Jordan

Ocyanthias martinicensis (Guichenot)

Paracentropristes pomospilus Ginsburg

.Paralabrax clathratus (Girard)

Paralabrax maculatofasciatus (Steindachner) Paralabrax nebulifer (Girard)

Paranthias furcifer (Valenciennes)

Petrometopon cruentatum (Lacépède)

...............Pikea mexicana Schultz

Polyprion americanus (Bloch and Schneider)

.Prionodes annularis (Günther)

Prionodes baldwini Evermann and Marsh

.Prionodes phoebe (Poey)

. Pronotogrammus aureorubens Longley

. Pseudogrammus brederi (Hildebrand) Roccus americanus (Gmelin)
.................. Prionodes notospilus (Longley)

\footnotetext{
1 In addition to the species listed, two are sufficiently important in the aport fishery near our area off Florida to warrant mention: misty grouper, Epinephelus mystocinus (Poey); yellowmouth grouper Mycteroperca interstitialis (Poey).
} 
White bass. ............. F

Yellow bass. . . . . . . . . . F F

Striped bass.

Freckled soapfish.......... A

Soapfish............... A

Spotted soapfish........... A

Belted sandfish........... A

Pygmy sea bass............. A

Chalk bass ............... A

Giant sea bass.............. P

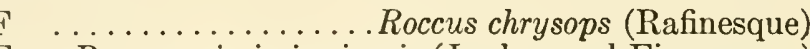
F . .Roccus mississippiensis (Jordan and Eigenmann) .................Rypticus arenatus Cuvier ...... Rypticus saponaceus (Bloch and Schneider)

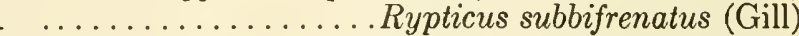
................Serranellus subligarius (Cope) A.............. Serraniculus pumilio Ginsburg A ................ Serranus tortugarum Longley

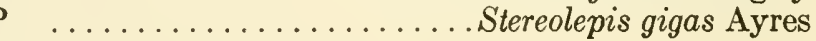

Lobotidae-tripletails

A Lobotes surinamensis (Bloch)

Lutjanidae-snappers

Black snapper. . . . . . . . . . .
A
A
Apsilus dentatus Guichenot
A
Etelis oculatus (Valenciennes)
A
A
A
A
A
A
A
A
A
A
A
A Lutjanus analis (Cuvier)
. Lutjanus apodus (Walbaum)
$\ldots \ldots \ldots \ldots$ Lutjanus blackfordi Goode and Bean
Lutjanus campechanus (Poey)
Lutjanus cyanopterus (Cuvier)
Lutjanus griseus (Linnaeus)
Lutjanus jocu (Bloch and Schneider)
Lutjanus synagris (Linnaeus)
Lutjanus vivanus (Cuvier)
Ocyurus chrysurus (Bloch)
Pristipomoides andersoni Ginsburg
. Rhomboplites aurorubens (Cuvier)

Queen snapper.

Schoolmaster.

Dog snapper.

Lane snapper.

Silk snapper. .

Wenchman

Vermilion snapper.

Priacanthidae--bigeyes

Bigeye..................

Glasseye snapper.

Short bigeye.

Popeye catalufa
A

$\mathrm{A}$

A

$\mathbf{P}$
Priacanthus arenatus Cuvier

$\ldots \ldots \ldots \ldots \ldots \ldots \ldots \ldots \ldots \ldots$ Priacanthus cruentatus (Lacépède)

Pristigenys serrula (Gilbert)

Apogonidae-cardinalfishes

Bronze cardinalfish

Bridle cardinalfish.

Barred cardinalfish

Freckled cardinalfish

Whitestar cardinalfish.

Flamefish .

Dusky cardinalfish.

Pale cardinalfish.

Twospot cardinalfish

Sawcheek cardinalfish

Conchfish

Belted cardinalfish
A

A

A

A

A

A

A

A

A

A

A

A
Apogon alutus Jordan and Gilbert

$\ldots \ldots \ldots \ldots \ldots \ldots$ A pogon aurolineatus (Mowbray) A pogon conklini (Silvester) . Apogon lachneri Böhlke . Apogon maculatus (Poey) Apogon pigmentarius (Poey) Apogon planifrons Longley and Hildebrand A pogon pseudomaculatus Longley A pogon quadrisquamatus Longley Apogon stellatus (Cope) Apogon townsendi (Breder) 
Centrarchidae - sunfishes

Mud sunfish............ F

Roanoke bass............ F

Rock bass................ F

Sacramento perch........... F

Flier................. F

Warmouth............ F

Everglades pygmy sunfish...... F F

Okefenokee pygmy sunfish..... F F

Banded pygmy sunfish........ F

Blackbanded sunfish.......... F

Bluespotted sunfish . . . . . . . . F F

Banded sunfish ............ F

Redbreast sunfish ........... F

Green sunfish.............. F F

Pumpkinseed.............. F

Orangespotted sunfish ........ F

Bluegill............... F F

Dollar sunfish ............ F

Longear sunfish............. F

Redear sunfish.............. F

Spotted sunfish............ F

Bantam sunfish............ F

Redeye bass.............. F

Smallmouth bass............ F

Suwannee bass............. F

Spotted bass............... F

Largemouth bass............. F F

Guadalupe bass............ F

White crappie............ F

Black crappie............. F
Acantharchus pomotis (Baird)

Ambloplites cavifrons Cope

Ambloplites rupestris (Rafinesque)

Archoplites interruptus (Girard)

Centrarchus macropterus (Lacépède)

Chaenobryttus gulosus (Cuvier)

. Elassoma evergladei Jordan

Elassoma okefenokec Böhlke

Elassoma zonatum Jordan

Enneacanthus chaetodon (Baird)

Enneacanthus gloriosus (Holbrook)

Enneacanthus obesus (Girard)

Lepomis auritus (Linnaeus)

Lepomis cyanellus Rafinesque

Lepomis gibbosus (Linnaeus)

Lepomis humilis (Girard)

Lepomis macrochirus Rafinesque

Lepomis marginatus (Holbrook)

. Lepomis megalotis (Rafinesque)

Lepomis microlophus (Günther)

Lepomis punctatus (Valenciennes)

Lepomis symmetricus Forbes

Micropterus coosae Hubbs and Bailey

Micropterus dolomicui Lacépède

Micropterus notius Bailey and Hubbs

Micropterus punctulatus (Rafinesque)

Micropterus salmoides (Lacépède)

Micropterus treculi (Vaillant and Bocourt)

Pomoxis annularis Rafinesque

Pomoxis nigromaculatus (LeSueur)

\section{Percidae - perches}

Crystal darter

Naked sand darter. . . . . . . . . . .

Western sand darter..........

Eastern sand darter..........

Scaly sand darter. ...........

Sharphead darter. . . . . . . . . . .

Mud darter. . . . . . . . . . . . . . .

Cumberland snubnose darter....

Scalyhead darter.

Greenside darter. . . . . . . . . . . . . .

Blenny darter. . . . . . . . . . . . .

Rainbow darter..............

Bluebreast darter. . . . . . . . . . .

Bluntnose darter. . . . . . . . . . .

Ashy darter. . . . . . . . . . . . . .

Carolina darter..............

Coosa darter. .

Arkansas darter. ........ Ammocrypta beani Jordan Ammocrypta clara Jordan and Meek . Ammocrypta pellucida (Baird) Ammocrypta vivax Hay Etheostoma acuticeps Bailey Etheostoma asprigene (Forbes) Etheostoma atripinne (Jordan) Etheostoma barratti (Holbrook) Etheostoma blennioides Rafinesque Etheostoma blennius Gilbert and Swain Etheostoma caeruleum Storer Etheostoma camurum (Cope) Etheostoma chlorosomum (Hay) Etheostoma cinereum Storer Etheostoma collis (Hubbs and Cannon) Etheostoma coosae (Fowler) Etheostoma cragini Gilbert Etheostoma duryi Henshall
Ammocrypta asprella (Jordan) 
Brown darter............ F F

Arkansas saddled darter...... F F

Iowa darter.............. F

Fantail darter............. F

Fountain darter............. F

Savannah darter.......... F

Swamp darter............. F

Slough darter............ F

Rio Grande darter. . . . . . . . . . F F

Tuckasegee darter.......... F

Harlequin darter............ F

Turquoise darter........... F

Blueside darter. ........... F

Greenbreast darter.......... F

Yoke darter............. F

Kanawha darter........... F

Stripetail darter............. F

Greenthroat darter.......... F F

Longfin darter............ F

Redband darter........... F

Spotted darter............. F

Pinewoods darter........... F F

Least darter.............. F F

Niangua darter........... F F

Johnny darter............ F F

Barcheek darter............ F

Finescale saddled darter....... F

Goldstripe darter............ F

Waccamaw darter.......... F

Riverweed darter........... F F

Cypress darter............ F

Stippled darter............ F

Orangebelly darter.......... F

Redline darter............. F

Rock darter.............. F

Arrow darter. . . . . . . . . . . F F

Saluda darter............. F

Maryland darter........... F

Sawcheek darter. . . . . . . . . . F F

Tennessee snubnose darter..... F F

Orangethroat darter.......... F

Spottail darter............ F

Speckled darter............ F

Gulf darter.............. F

Swannanoa darter............ F

Missouri saddled darter........ F F

Seagreen darter............ F

Tippecanoe darter........... F F

Tuseumbia darter.......... F F

Variegate darter............. F

Striped darter............. F F

Glassy darter............... F F

Redfin darter.............. F

Banded darter............. F
Etheostoma edwini (Hubbs and Camnon)

Etheostoma euzonum (Hubbs and Black)

Etheostoma exile (Girard)

Etheostoma flabellare Rafinesque

Etheostoma fonticola (Jordan and Gilbert)

Etheostoma fricksium Hildebrand Etheostoma fusiforme (Girard) Etheostoma gracile (Girard)

Etheostoma grahami (Girard) Etheostoma gutselli (Hildebrand)

Etheostoma histrio Jordan and Gilbert Etheostoma inscriptum (Jordan and Brayton) Etheostoma jessiae (Jordan and Brayton) Etheostoma jordani Gilbert Etheostoma juliae Meek Etheostoma kanawhae (Raney) Etheostoma kennicotti (Putnam) Etheostoma lepidum (Baird and Girard) Etheostoma longimanum Jordan Etheostoma luteovinctum Gilbert and Swain Etheostoma maculatum Kirtland Etheostoma mariae (Fowler) Etheostoma microperca Jordan and Gilbert Etheostoma nianguae Gilbert and Meek Etheostoma nigrum Rafinesque Etheostoma obeyense Kirseh Etheostoma osburni (Hubbs and Trautman) Etheostoma parvipinne Gilbert and Swain Etheostoma perlongum (Hubbs and Raney) Etheostoma podostemone Jordan and Jenkins Etheostoma proeliare (Hay)

Etheostoma punctulatum (Agassiz) Etheostoma radiosum (Hubbs and Black) Etheostoma rufilineatum (Cope) Etheostoma rupestre Gilbert and Swain Etheostoma sagitta (Jordan and Swain) Etheostoma saludae (Hubbs and Cannon) Etheostoma sellare (Radcliffe and Welch)

Etheostoma serriferum (Hubbs and Cannon) .Etheostoma simoterum (Cope) Etheostoma spectabile (Agassiz) Etheostoma squamiceps Jordan Etheostoma stigmaeum (Jordan) Etheostoma swaini (Jordan) .... Etheostoma swannanoa Jordan and Evermann ........ Etheostoma tetrazonum (Hubbs and Black) ... Etheostoma thalassinum (Jordan and Brayton) .....Etheostoma tippecanoe Jordan and Evermann .........Etheostoma tuscumbia Gilbert and Swain Etheostoma variatum Kirtland Etheostoma virgatum (Jordan) Etheostoma vitreum (Cope) Etheostoma whipplei (Girard) Etheostoma zonale (Cope) 
Backwater darter........... F

Yellow perch............ F

Yellow darter.

Logperch.

Channel darter

Piedmont darter

Bluestripe darter.

Gilt darter

Longhead darter

Blackside darter

Longnose darter.

Blackbanded darter

Stripeback darter.

Sharpnose darter.

Bronze darter.

Leopard darter.

Shield darter.

Slenderhead darter

Roanoke logperch

Dusky darter.

River darter.

Olive darter.

Stargazing darter

Sauger .

Walleye.

Blue pike
$\mathrm{F}$

F

F

F

F

F

F

F

F

F

F

F

F

F

F

F

F

F

F

$\mathrm{F}$

F

F

F

$\mathrm{F}$

F
Etheostoma zoniferum (Hubbs and Cannon) Perca flavescens (Mitchill)

Percina aurantiaca (Cope)

Percina caprodes (Rafinesque)

.Percina copclandi (Jordan)

Percina crassa (Jordan and Brayton)

Percina cymatotaenia (Gilbert and Meek)

Percina evides (Jordan and Copeland)

Percina macrocephala (Cope)

Percina maculata (Girard)

Percina nasuta (Bailey)

Percina nigrofasciata (Agassiz)

Percina notogramma (Raney and Hubbs)

Percina oxyrhyncha (Hubbs and Raney) Percina palmaris (Bailey)

Percina pantherina (Moore and Reeves) Percina peltata (Stauffer)

Percina phoxocephala (Nelson) Percina rex (Jordan and Evermann) . Percina sciera (Swain)

Percina shumardi (Girard)

Percina squamata (Gilbert and Swain)

Percina uranidea (Jordan and Gilbert)

Stizostedion canadense (Smith)

Stizostedion vitreum vitreum (Mitchill) . . Stizostedion vitreum glaucum Hubbs

Branchiostegidae-tilefishes

Blackline tilefish ........... A

Ocean whitefish........... P

Tilefish................ A

Sand tilefish

A

Caulolatilus cyanops Poey

Caulolatilus princeps (Jenyns)

Pomatomidae-bluefishes

Bluefish A

Pomatomus saltatrix (Linnaeus)

Rachycentridae - cobias

Cobia

A

Rachycentron canadum (Linnaeus)

Carangidae ${ }^{1}$-jacks, scads, and pompanos

African pompano............ A

Yellow jack. ............. A

Green jack................ P

Blue runner.............. A

Crevalle jack............. A A

Horse-eye jack............. A

Bar jack............... A

Bumper................ A

Mexican scad.............. $\mathrm{P}$

Mackerel scad.............. A

Round scad.

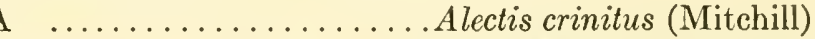

. Caranx bartholomaei Cuvier Caranx caballus Günther

.Caranx crysos (Mitchill)

Caranx hippos (Linnaeus)

.Caranx latus Agassiz

Caranx ruber (Bloch)

Chloroscombrus chrysurus (Linnaeus)

Decapterus hypodus Gill

Decapterus macarellus (Cuvier)

Decapterus punctatus (Agassiz)

\footnotetext{
1 An important sport fish, the black jack, Caranx lugubris Poey, occurs just out of our area and is landed at Florida por
} 
Rainbow runner.

Bluntnose jack

Pilotfish.

Roosterfish.

Leatherjacket

Bigeye scad

Lookdown

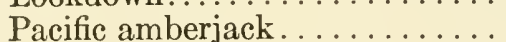

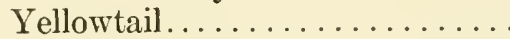

Greater amberjack...........

Almaco jack.

Lesser amberjack.

Banded rudderfish

Pompano.

Permit.

Palometa.

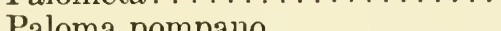

Gafftopsail pompano..........

Rough scad...............

Jack mackerel

Cottonmouth jack

Pacific moonfish

Atlantic moonfish.
A

A

A-P

$\mathrm{P}$

A-P

A

A

$\mathrm{P}$

$\mathrm{P}$

A

A

A

A

A

A

A

$\mathrm{P}$

$\mathrm{P}$

A

$\mathrm{P}$

A

$\mathrm{P}$

A
Elagatis bipinnulatus (Quoy and Gaimard) $\ldots \ldots \ldots \ldots$ Hemicaranx amblyrhynchus (Cuvier) ......................... ........ Oligoplites saurus (Bloch and Schneider)

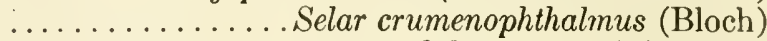
Selene vomer (Linnaeus) . Seriola colburni (Evermann and Clark) Seriola dorsalis (Gill) Seriola dumerili (Risso) Seriola falcata Valenciennes Seriola fasciata (Bloch) Seriola zonata (Mitchill) Trachinotus carolinus (Linnaeus) Trachinotus falcatus (Linnaeus) Trachinotus glaucus (Bloch) Trachinotus paitensis Cuvier Trachinotus rhodopus (Gill) .Trachurus lathami Nichols Trachurus symmetricus (Ayres) Uraspis heidi Fowler Vomer declivifrons Meek and Hildebrand Vomer setapinnis (Mitchill)

Coryphaenidae-dolphins

Pompano dolphin

Dolphin . A A-P Coryphaena equisetis Linnaeus Coryphaena hippurus Linnaeus

Bramidae-pomfrets

Pomfret. A-P

Bigscale pomfret

A-P .......Brama rayi (Bloch) Taractes longipinnis (Lowe)

\section{Gerridae-mojarras}

Irish pompano........... A Diapterus olisthostomus (Goode and Bean)

Striped mojarra........... A A

Spotfin mojarra........... A-P

Silver jenny . . . . . . . . . . .

Bigeye mojarra. . . . . . . . . .

Mottled mojarra. .............

Flagfin mojarra.............

Slender mojarra.............

Yellowfin mojarra........... A
A ..........Eucinostomus gula (Quoy and Gaimard)

A ..............Ecinostomus havana (Nichols)

A $\quad \ldots \ldots \ldots \ldots \ldots \ldots$ Eucinostomus lefroyi (Goode)

A ..........Eucinostomus melanopterus (Bleeker)

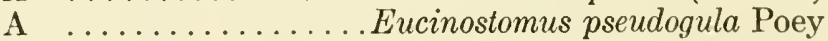

A .................... cinereus (Walbaum)

Pomadasyidae-grunts

Sargo............... P

Black margate............ A

Porkfish................. A

Tomtate.............. A

Smallmouth grunt.......... A

Barred grunt............. A

Margate............... A

Black grunt............. A

Caesar grunt
..........Anisotremus davidsoni (Steindachner)

............Anisotremus surinamensis (Bloch)

............Anisotremus virginicus (Linnaeus)

.............Bathystoma aurolineatum (Cuvier)

A ...........Brachygenys chrysargyrea (Günther)

A ........................

..................... Haemulon album Cuvier

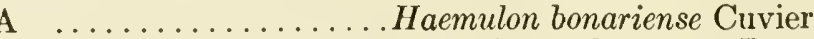

A ....................emulon carbonarium Poey 
French grunt............

Spanish grunt............ A

Cottonwick............... A

Sailors choice

White grunt.

Bluestriped grunt.

Pigfish.

Burro grunt

Salema
A

A

A

A

$\mathrm{P}$

Haemulon flavolineatum (Desmarest) Haemulon macrostomum Günther Haemulon melanurum (Linnaeus) Haemulon parrai (Desmarest) Haemulon plumieri (Lacépède) Haemulon sciurus (Shaw) Orthopristis chrysopterus (Linnaeus) Pomadasys crocro (Cuvier) Xenistius californiensis (Steindachner)

\section{Sciaenidae-drums}

Freshwater drum .......... F

Silver perch . . . . . . . . . . . . A

Bairdiella

Black croaker.

Sand seatrout.

Spotted seatrout

White seabass

Silver seatrout.

Shortfin corvina

Weakfish

Orangemouth corvina.........

Cubbyu...................

Jackknife-fish . . . . . . . . . . .

Striped drum.

Spotted drum

White croaker

Banded drum

Spot

Southern kingfish

Minkfish

Gulf kingfish

Northern kingfish

California corbina.

Atlantic croaker.

Reef croaker.

Catalina croaker

Black drum.

Spotfin croaker

Red drum....

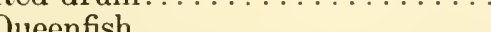

Queenfish

Star drum.

Sand drum.

Yellowfin croaker

Blue croaker.

Yellow goatfish

Red goatfish

Mexican goatfish

A $\mathrm{A}$
Aplodinotus grunniens Rafinesque Bairdiella chrysura (Lacépède)

Bairdiella icistius (Jordan and Gilbert) . Cheilotrema saturnum (Girard) Cynoscion arenarius Ginsburg Cynoscion nebulosus (Cuvier) ...Cynoscion nobilis (Ayres) Cynoscion nothus (Holbrook) Cynoscion parvipinnis Ayres Cynoscion regalis (Bloch and Schneider) Cynoscion xanthulus Jordan and Gilbert Equetus acuminatus (Bloch and Schneider) Equetus lanceolatus (Linnaeus)

Equetus pulcher (Steindachner) Equetus punctatus (Bloch and Schneider) Genyonemus lineatus (Ayres) Larimus fasciatus Holbrook Leiostomus xanthurus Lacépède Menticirrhus americanus (Linnaeus) Menticirrhus focaliger Ginsburg Menticirrhus littoralis (Holbrook) A .....Menticirrhus saxatilis (Bloch and Schneider) P ................ Menticirrhus undulatus (Girard) A ..................

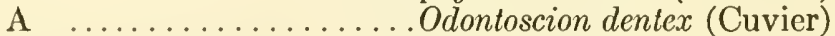
P ............... Ophioscion thompsoni (Hubbs)

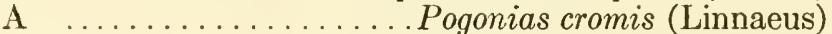
P ............Roncador stearnsi (Steindachner)

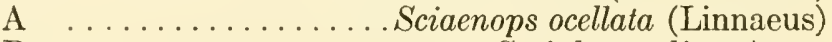

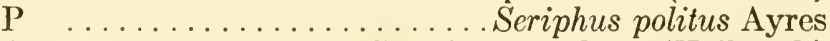

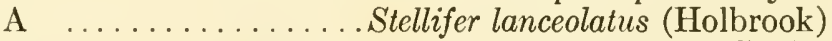
A .................. Umbrina coroides Cuvier P ........... Umbrina roncador Jordan and Gilbert A ........Vacuoqua sialis (Jordan and Eigenmann)

Mullidae-goatfishes . Mulloidichthys martinicus (Cuvier) Mullus auratus Jordan and Gilbert $\mathrm{P}$ Pseudupeneus dentatus (Gill)

\footnotetext{
${ }^{1}$ In our area confined to Salton Sea.
} 
Spotted goatfish

Dwarf goatfish
A

A
Pseudupeneus maculatus (Bloch) Upeneus parvus Poey

Sparidae-porgies

Sheepshead

A . Archosargus probatoccphalus (Walbaum)

Sea bream.............. A

Grass porgy ............... A

Jolthead porgy ............. A

Pacific porgy ............. P

Saucereye porgy ........... A

Whitebone porgy........... A

Sheepshead porgy........... A

Littlehead porgy............ A

Silver porgy............ A

Spottail pinfish............ A

Pinfish................ A

Red porgy ............. A

Longspine porgy ........... A

Scup................ A Archosargus rhomboidalis (Linnaeus) .Calamus arctifrons Goode and Bean Calamus bajonado (Bloch and Schneider) . Calamus brachysomus (Lockington) .Calamus calamus (Valenciennes) Calamus leucosteus Jordan and Gilbert .Calamus penna (Valenciennes) Calamus proridens Jordan and Gilbert Diplodus argentcus (Valenciennes) Diplodus holbrooki (Bean) Lagodon rhomboides (Linnaeus) Pagrus sedecim Ginsburg Stenotomus caprinus Bean

Pempheridae-sweepers

Glassy sweeper

A ......Pempheris schomburgki Müller and Trosehel

\section{Seorpidae-halfmoons}

Halfmoon.............. P Medialuna californiensis (Steindachner)

Kyphosidae-sea chubs

Zebra perch............. P

Yellow chub............. A

Hermosilla azurea Jenkins and Evermann

Bermuda chub.

A Kyphosus incisor (Cuvier) Kyphosus sectatrix (Linnaeus)

Girellidae-nibblers

Opaleye. $\mathrm{P}$ Girella nigricans (Ayres)

Ephippidae-spadefishes

Atlantic spadefish

A Chaetodipterus faber (Broussonet) Pacific spadefish.

\section{Chaetodontidae-butterflyfishes}

Cherubfish . .

Bank butterflyfish

Foureye butterflyfish

Spotfin butterflyfish

Reef butterflyfish

Banded butterflyfish.

Blue angelfish

Queen angelfish

Rock beauty

French angelfish

Gray angelfish

Longsnout butterflyfish
A ...........entropyge argi Woods and Kanazawa A .................... Chaetodon aya Jordan A A A A A A A $\mathrm{A}$ A A
Chaetodon capistratus Linnaeus . Chactodon occllatus Bloch Chactodon sedentarius Poey Chaetodon striatus Linnaeus Holacanthus bermudensis (Goode) Holacanthus ciliaris (Linnacus) .Holacanthus tricolor (Bloch) Pomacanthus arcuatus (Linnaeus) Pomacanthus aurcus (Bloch) Prognathodes aculeatus (Poey) 


\section{Cichlidae-cichlids}

Velvet cichlid............. F

F .............Astronotus ocellatus (Agassiz) Rio Grande perch.......... F ..... Cichlasoma cyanoguttatum (Baird and Girard)

\section{Embiotocidae-surfperches}

Barred surfperch

Calico surfperch

Redtail surfperch.

Kelp perch.

Shiner perch.

Island seaperch

Black perch

Striped seaperch

Spotfin surfperch

Walleye surfperch

Silver surfperch.

Rainbow seaperch.

Tule perch

Reef perch.

Dwarf perch. .

Sharpnose seaperch

White seaperch

Rubberlip seaperch.

Pile perch.

Pink seaperch .
$\mathrm{P}$

$\mathrm{P}$

$\mathrm{P}$

$\mathrm{P}$

$\mathrm{P}-\mathrm{F}$

$\mathrm{P}$

$\mathrm{P}$

$\mathrm{P}$

$\mathrm{P}$

$\mathrm{P}$

$\mathrm{P}$

$\mathrm{P}$

$\mathrm{F}$

$\mathrm{P}$

$\mathrm{P}$

$\mathrm{P}$

$\mathrm{P}$

$\mathrm{P}$

$\mathrm{P}$

$\mathrm{P}$
Amphistichus argenteus Agassiz

Amphistichus koelzi (Hubbs) . Amphistichus rhodoterus (Agassiz)

Brachyistius frenatus Gill

Cymatogaster aggregata Gibbons

Cymatogaster gracilis Tarp

Embiotoca jacksoni Agassiz

Embiotoca lateralis Agassiz

Hyperprosopon anale Agassiz

Hyperprosopon argenteum Gibbons

Hyperprosopon ellipticum (Gibbons)

.Hypsurus caryi (Agassiz)

Hysterocarpus traski Gibbons

Micrometrus aurora (Jordan and Gilbert)

Micrometrus minimus (Gibbons)

Phanerodon atripes (Jordan and Gilbert)

Phanerodon furcatus Girard

. Rhacochilus toxotes Agassiz

Rhacochilus vacca (Girard)

Zalembius rosaceus (Jordan and Gilbert)

\section{Pomacentridae-damselfishes}

Sergeant major

Night sergeant.

Blue chromis

Yellowtail reef-fish

Gray reef-fish

Brown chromis

Blacksmith.

Dusky damselfish

Beaugregory.....

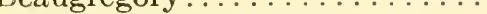

Bicolor damselfish.... . . . . . . . .

Threespot damselfish . . . . . . . . .

Cocoa damselfish.............

Garibaldi .

Yellowtail damselfish
A A A A A A $\mathrm{P}$ A A

A

A

A $\mathrm{P}$ A
Abudefduf saxatilis (Linnaeus) .......... Abudefduf taurus (Müller and Troschel) Chromis cyanea (Poey) Chromis enchrysurus Jordan and Gilbert Chromis insolatus (Cuvier) Chromis multilincata (Guichenot) Chromis punctipinnis (Cooper) Eupomacentrus dorsopunicans (Poey) Eupomacentrus leucostictus (Müller and Troschel) Eupomacentrus partitus (Poey)

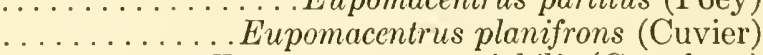
.......... Eupomacentrus variabilis (Castelnau) ................ Hypsypops rubicunda (Girard) Microspathodon chrysurus (Cuvier)

\section{Labridae - wrasses}

Spotfin hogfish

Spanish hogfish

Creole wrasse.

Red hogfish .

Dwarf wrasse.

Greenband wrasse

Slippery dick.

Painted wrasse.

Yellowhead wrasse
A

A

$\mathrm{A}$

A

A

A

A

A

A
Bodianus pulchellus (Poey)

. Bodianus rufus (Linnaeus) Clepticus parrai (Bloch and Schneider) Decodon puellaris (Poey) Halichocres bathyphilus Beebe and Tee-Van Halichoeres bivittatus (Bloch) Halichoeres caudalis (Poey) Halichoeres garnoti (Valenciennes) 
Clown wrasse............. A

Blackear wrasse............ A

Puddingwife............... A

Rock wrasse.............. P

Hogfish.................. A

Señorita............... P

California sheephead......... P

Tautog. . ............... A

Cunner................ A

Bluehead............... A

Rosy razorfish ............. A

Pearly razorfish............ A

Green razorfish . . . . . . . . . . A
Halichoeres maculipinna (Müller and Troschel) Halichoeres poeyi (Steindachner) . Halichoeres radiatus (Linnaeus) Halichoeres semicinctus (Ayres)

Lachnolaimus maximus (Walbaum) Oxyjulis californica (Günther) .Pimelometopon pulchrum (Ayres) Tautoga onitis (Linnaeus) Tautogolabrus adspersus (Walbaum) . Thalassoma bifasciatum (Bloch) Xyrichthys martinicensis Valenciennes .Xyrichthys psittacus (Linnaeus) .Xyrichthys ventralis Bean

\section{Scaridae-parrotfishes}

Bluelip parrotfish........... A

Emerald parrotfish........... A

Purple parrotfish............ A

Blue parrotfish............. A

Striped parrotfish .......... A

Rainbow parrotfish.......... A

Queen parrotfish........... A

Redband parrotfish........... A

Spotfin parrotfish ............ A

Redtail parrotfish.......... A

Bucktooth parrotfish........ A

Redfin parrotfish........... A

Stoplight parrotfish.......... A
Cryptotomus roseus Cope Nicholsina usta (Valenciennes) Scarus coelestinus Valenciennes Scarus coeruleus (Bloch) Scarus croicensis Bloch Scarus guacamaia Cuvier Scarus vetula Bloch and Schneider Sparisoma aurofrenatum (Valenciennes) . Sparisoma axillare Steindachner ...Sparisoma chrysopterum (Bloch and Schneider) . . Sparisoma radians (Valenciennes) Sparisoma rubripinne (Valenciennes) .Sparisoma viride (Bonnaterre)

Acanthuridae-surgeonfishes

Ocean surgeon. . ........... A

Doctorfish ............... A

Blue tang ............. A

Gulf surgeonfish.......... A
Acanthurus bahianus Castelnau ................. Acanthurus coeruleus Bloch and Schneider A ........Acanthurus randalli Briggs and Caldwell

Gempylidae-snake mackerels

Snake mackerel............ A

Escolar. . . . . . . . . . . . . . A-P

Oilfish ............... A-P
Gempylus serpens Cuvier Lepidocybium flavobrunneum (Smith) Ruvettus pretiosus Cocco

Trichiuridae-cutlassfishes

Scabbardfish............. P

Atlantic cutlassfish.......... A

Lepidopus xantusi Goode and Bean

Pacific cutlassfish

$\mathrm{A}$ Trichiurus lepturus Linnaeus Trichiurus nitens Garman

Scombridae-mackerels and tunas

Wahoo

Frigate mackerel............. A

Little tuna................ A

Black skipjack............ P

Skipjack tuna.............. A-P

Wavyback skipjack
A .........................

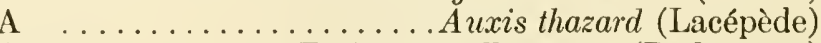

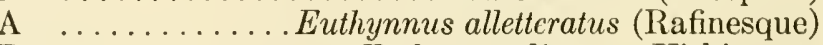

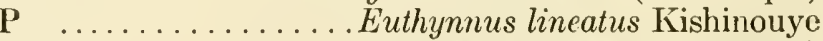

. . . . . . . ....... Euthynnus pelamis (Linnaeus) 
Pacific bonito............. P

Striped bonito............ A

Atlantic bonito............ A

Chub mackerel. . . . . . . . . . A

Pacific mackerel. . . . . . . . . . . P

Atlantic mackerel........... A

King mackerel............ A

Monterey Spanish mackerel.... . P

Spanish mackerel........... A

Cero.................. A

Albacore.............. A-P

Yellowfin tuna............ A-P

Blackfin tuna............. A

Bigeye tuna............. A-P

Bluefin tuna............ A-P

Sarda chiliensis (Cuvier)

.......Sarda orientalis (Temminck and Schlegel)

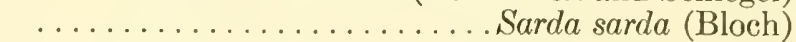

............................ Scomber colias Gmelin

........................ Scomber japonicus Houttuyn

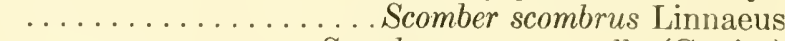

........ Scomberomorus cavalla (Cuvier)

........... Scomberomorus concolor (Lockington)

.......... Scomberomorus maculatus (Mitchill)

P .............. Thunnus alaiunga (Bonnaterre)

Thunnus albacares (Bonnaterre)

. . . . . . . . . . . . . . . .

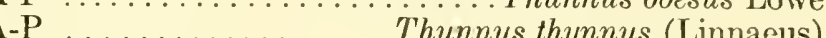

Luvaridae-louvars

Louvar. . . . . . . . . . . . . A-P

Luvarus imperialis Rafinesque

Istiophoridae ${ }^{1}$-billfishes

Atlantic sailfish............ A

White marlin............. A

Striped marlin. ............ P

Blue marlin . . . ......... A

Shortbill spearfish.......... P

Longbill spearfish........... A

Istiophorus albicans (Latreille)

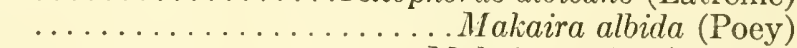

............................. audax (Phillipi)

...................... Maira nigricans Lacépède

A ............ Tetrapturus angustirostris Tanaka

Xiphiidae-swordfishes

Swordfish.............. A-P

Xiphias gladius Linnaeus

Eleotridae-sleepers

Fat sleeper.............. A-F

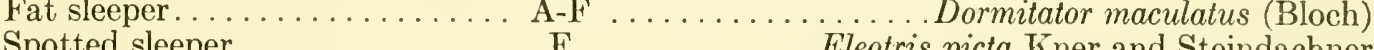

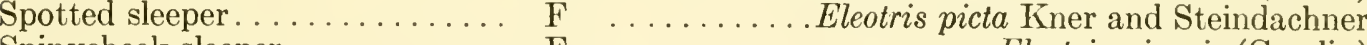

Spinycheek sleeper......... F . . . . . . . . . . . Eleotris pisonis (Gmelin)

Emerald sleeper............ A . . . . . . . . Erotelis smaragdus (Valenciennes)

Masked sleeper............ A ........ Eviota personata Jordan and Thompson

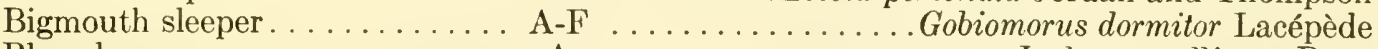

Blue sleeper............. A

Ioglossus calliurus Bean

Gobiidae-gobies

River goby............ A-F ........... Awaus tajasica (Lichtenstein)

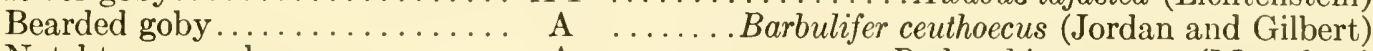

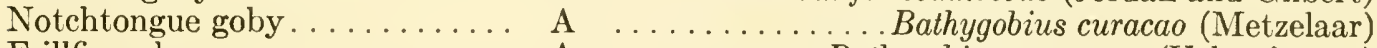

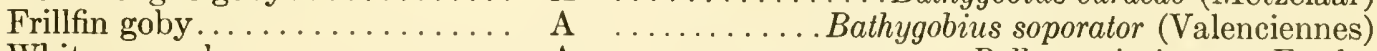

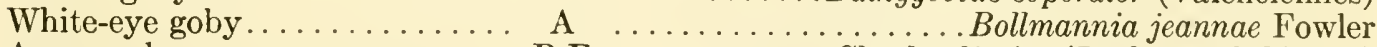

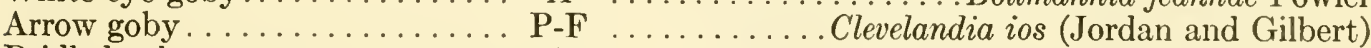

Bridled goby ............. A $\ldots \ldots \ldots \ldots \ldots$ Coryphopterus glaucofraenum Gill

Bluespot goby ........... P $\quad \ldots \ldots \ldots \ldots \ldots$. Coryphopterus nicholsi (Bean)

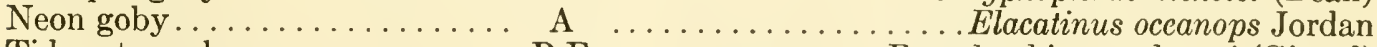

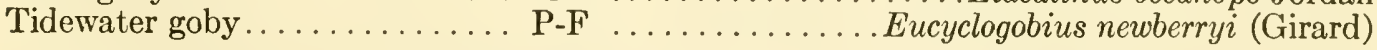

\footnotetext{
1 The following species, not yet recorded from our area, occur south of the California border in the Pacific: Pacific sailfish, Istiophorus greyi Jordan and Evermann; black marlin, Makaira indica (Cuvier); and blue marlin, Makaira nigricans Lacépede.
} 
Sponge goby.............

Lyre goby................

Tiger goby .

Longjaw mudsucker.

Goldspot goby.

Violet goby

Darter goby...

Slim goby

Sharptail goby

Longtail goby.

Highfin goby

Freshwater goby .

Emerald goby.

Marked goby

Spottail goby

Naked goby.

Seaboard goby

Whiteline goby

Twoscale goby.

Orangespotted goby

Code goby .

Paleback goby.

Cheekspot goby

Bay goby

Halfblind goby .

Crested goby .

Bluebanded goby .

Zebra goby

Seminole goby.

Clown goby.

Banner goby.

Green goby.

Shadow goby

Smallmouth goby.

Blind goby.
A

A

A

$\mathrm{P}-\mathrm{F}$

A

A

A

A

A

$\mathrm{P}$

A

A-F

A

A

A

A

A

A

A

A

A

A

$\mathrm{P}$

$\mathrm{P}$

$\mathrm{P}$

A

P

P

A

A

A

A

$\mathrm{P}$

A

$\mathrm{P}$
Evermannichthys spongicola (Radcliffe) .Evorthodus lyricus (Girard) Garmannia macrodon (Beebe and Tee-Van) Gillichthys mirabilis Cooper Gnatholepis thompsoni Jordan Gobioides broussonneti Lacépède Gobionellus boleosoma (Jordan and Gilbert) . Gobionellus gracillimus Ginsburg Gobioncllus hastatus Girard Gobionellus longicaudus (Jenkins and Evermann) Gobionellus oceanicus (Pallas) Gobionellus shufeldti (Jordan and Evermann) Gobionellus smaragdus (Valenciennes) Gobionellus stigmaticus (Poey) Gobionellus stigmaturus (Goode and Bean) . Gobiosoma bosci (Lacépède) Gobiosoma ginsburgi Hildebrand and Schroeder . .Gobiosoma horsti Metzelaar Gobiosoma longipala Ginsburg Gobiosoma longum Nichols Gobiosoma robustum Ginsburg Gobulus myersi Ginsburg

Ilypnus gilberti (Eigenmann and Eigenmann) Lepidogobius lepidus (Girard) Lethops connectens Hubbs Lophogobius cyprinoides (Pallas) Lythrypnus dalli (Gilbert) Lythrypnus zebra (Gilbert) Microgobius carri Fowler Microgobius gulosus (Girard) . Microgobius microlepis Longley and Hildebrand . Microgobius thalassinus (Jordan and Gilbert) Quietula y-cauda (Jenkins and Evermann) ....................... Risor ruber (Rosén) . .Typhlogobius californiensis Steindachner

Microdesmidae-wormfishes

Pugjaw wormfish.

Pink wormfish.
A

$\mathrm{A}$
Microdesmus floridanus (Longley) Microdesmus longipinnis (Weymouth)

Scorpaenidae-scorpionfishes and rockfishes

Blackbelly rosefish .

Spinycheek scorpionfish

Longsnout scorpionfish.

Scalyhead scorpionfish.

Highfin scorpionfish.

Longfin scorpionfish

Red scorpionfish

Goosehead scorpionfish

Barbfish.

Smoothhead scorpionfish

Hunchback scorpionfish

Lionfish.
A

A

A

A

A

A

A

A

A

A

A

A
Helicolenus dactylopterus (De la Roche) .Neomerinthe pollux (Poey) Pontinus castor Poey Pontinus longispinis Goode and Bean ... Pontinus rathbuni Goode and Bean . Scorpaena agassizi Goode and Bean Scorpacna atlantica Nichols and Breder . Scorpaena bergi Evermann and Marsh . Scorpacna brasiliensis Cuvier - Scorpaena calcarata Goode and Bean Scorpaena dispar Longley and Hildebrand . Scorpaena grandicornis Cuvier 
California scorpionfish........ P P

Mushroom scorpionfish........ A

Finescale scorpionfish ......... . A

Spotted scorpionfish.......... A

Reef scorpionfish............ A

Redfish or ocean perch........ A

Blackthroat rockfish.......... P P

Pacific ocean perch.......... P

Kelp rockfish.............. P

Brown rockfish............ P

Aurora rockfish ............. P

Silvergray rockfish . ........ P P

Gopher rockfish............. P

Copper rockfish............ P P

Greenspotted rockfish......... . P

Black-and-yellow rockfish . ..... . P

Dusky rockfish............ P

Starry rockfish............ P

Blackmouth rockfish......... P

Calico rockfish.............. P

Splitnose rockfish.......... P P

Greenstriped rockfish......... P P

Puget Sound rockfish........ . P

Widow rockfish............ P

Pink rockfish ............ P

Yellowtail rockfish.......... P

Bronzespotted rockfish......... P P

Chilipepper............... . P

Rosethorn rockfish.......... P

Squarespot rockfish.......... P P

Shortbelly rockfish.......... P

Cow rockfish ............. P

Coral-red rockfish........... P

Quillback rockfish.......... P

Black rockfish............ P

Blackgill rockfish........... P

Vermilion rockfish.......... P

Blue rockfish............. P

China rockfish............ P P

Tiger rockfish.............. P

Speckled rockfish........... P P

Bocaccio............... P

Canary rockfish............ P

Northern rockfish............ P P

Redstripe rockfish........... P

Grass rockfish............. . P

Swordspine rockfish . . . . . . . P

Rosy rockfish............. P

Rasphead rockfish........... P

Flag rockfish . . . . . . . . . P

Stripetail rockfish . . . . . . . . . P P

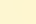

Scorpaena microlepis Gunter

Scorpaenodes caribbaeus Meek and Hildebrand

. Sebastes marinus (Linnaeus)

Sebastodes ${ }^{1}$ aleutianus Jordan and Evermann

.................. Sebastodes alutus (Gilbert)

Sebastodes atrovirens (Jordan and Gilbert)

Sebastodes auriculatus (Girard)

. Sebastodes aurora (Gilbert)

Sebastodes brevispinis (Bean)

Sebastodes carnatus (Jordan and Gilbert)

Sebastodes caurinus (Richardson)

Sebastodes chlorostictus (Jordan and Gilbert)

Sebastodes chrysomelas (Jordan and Gilbert)

Sebastodes ciliatus (Tilesius)

Sebastodes constellatus (Jordan and Gilbert)

Sebastodes crameri Jordan

Sebastodes dalli (Eigenmann and Beeson)

Sebastodes diploproa (Gilbert)

Sebastodes elongatus (Ayres)

. Sebastodes emphaeus Starks

Sebastodes cntomelas (Jordan and Gilbert)

Sebastodes cos (Eigenmann and Eigenmann) Scbastodes flavidus Ayres

Sebastodes gilli Eigenmann and Eigenmann

Sebastodes goodei Eigenmann and Eigenmann

............... Sebastodes helvomaculatus (Ayres)

............... Sebastodes hopkinsi Cramer

Sebastodes jordani Gilbert

Sebastodes levis (Eigenmann and Eigenmann)

. Sebastodes macdonaldi (Eigenmann and Beeson)

......... Sebastodes maliger (Jordan and Gilbert)

Sebastodes melanops (Girard)

Sebastodes melanostomus

Eigenmann and Eigenmann

Sebastodes miniatus (Jordan and Gilbert)

Sebastodes mystinus (Jordan and Gilbert)

Sebastodes nebulosus (Ayres)

Sebastodes nigrocinctus (Ayres)

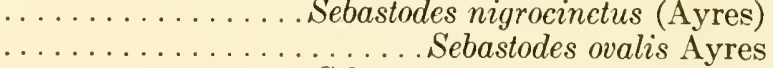

Sebastodes paucispinis (Ayres)

Sebastodes pinniger (Jordan and Gilbert)

Sebastodes polyspinis (Taranetz and Moiseev)

... Sebastodes proriger (Jordan and Gilbert)

. Sebastodes rastrelliger (Jordan and Gilbert)

. Sebastodes rhodochloris (Jordan and Gilbert)

. Sebastodes rosaceus Jordan and Gilbert

Sebastodes ruberrimus Cramer

Sebastodes rubrivinctus (Jordan and Gilbert)

. Sebastodes saxicola (Gilbert)

1 The spccies here assigned to Sebastodes are referred by some authors to Seljastes. 
Halfbanded rockfish......... P

Olive rockfish.............. P

Treefish.

Honeycomb rockfish .

Whitebelly rockfish. .

Pygmy rockfish............ P

Sharpchin rockfish.......... P

Shortspine channel rockfish..... P

Longspine channel rockfish..... P P
Sebastodes semicinctus Gilbert . Sebastodes serranoides

Eigenmann and Eigenmann Sebastodes serriceps (Jordan and Gilbert) Sebastodes umbrosus (Jordan and Gilbert) Sebastodes vexillaris (Jordan and Gilbert) . Sebastodes wilsoni Gilbert Sebastodes zacentrus (Gilbert) . Sebastolobus alascanus Bean . Sebastolobus altivelis Gilbert

Triglidae-searobins

Shortfin searobin........... A

Streamer searobin........... A

Horned searobin ............ A

Slenderhead searobin......... A

Armored searobin ............. A

Spiny searobin............ A

Sicklefin searobin............ A

Northern searobin........... A

Striped searobin. . . . . . . . . .

Barred searobin.

Bandtail searobin

Mexican searobin .

Blackwing searobin

Bluespotted searobin

Blackfin searobin.

Leopard searobin

Shortwing searobin

Lumptail searobin

Bighead searobin .
A .................... Bellator brachychir (Regan)

Bellator egretta (Goode and Bean)

Bellator militaris (Goode and Bean)

Peristedion imberbe (Poey)

Peristedion miniatum Goode

Prionotus alatus Goode and Bean Prionotus beani Goode Prionotus carolinus (Linnaeus) Prionotus evolans (Linnaeus)

Prionotus martis Ginsburg

A $\quad \ldots \ldots \ldots \ldots$ Prionotus ophryas Jordan and Swain

A .........Prionotus pectoralis Nichols and Breder A ........Prionotus roseus Jordan and Evermann

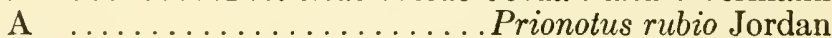
A $\quad \ldots . . . . .$. Prionotus scitulus Jordan and Gilbert

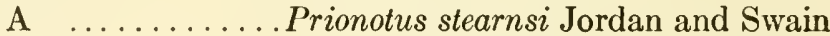
P ...........Prionotus stephanophrys Lockington

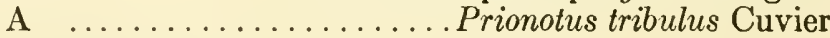

Anoplopomatidae-sablefishes

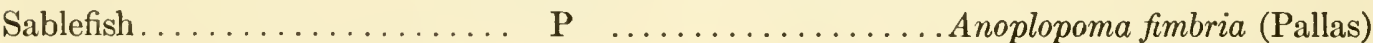
Skilfish............. P $\quad \ldots \ldots \ldots \ldots \ldots$ Erilepis zonifer (Lockington)

Hexagrammidae-greenlings

Kelp greenling............ P . . . . . . . Hexagrammos decagrammus (Pallas)

Masked greenling .......... $\mathrm{P} \quad \ldots \ldots \ldots \ldots$ Hexagrammos octogrammus (Pallas)

Whitespotted greenling ...... $\quad$ P ............Hexagrammos stelleri Tilesius

Rock greenling............ P .......... Pexagrammos superciliosus (Pallas)

Lingcod.............. P $\quad \ldots \ldots \ldots \ldots$ Ophiodon elongatus Girard

Painted greenling ......... P $\quad \ldots \ldots \ldots \ldots \ldots \ldots \ldots \ldots$ Oxylebius pictus Gill

Atka mackerel............ P ........Pleurogrammus monopterygius (Pallas)

Zaniolepidae-combfishes

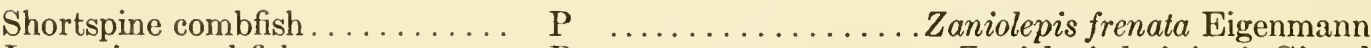
Longspine combfish ........ P . . . . . . . . . . Zaniolepis latipinnis Girard

Cottidae ${ }^{1}$-sculpins

Hookear sculpin . Artedius corallinus (Hubbs) 
Roughcheek sculpin .

Padded sculpin.

Spurcheek sculpin.

Scalyhead sculpin .

Smoothhead sculpin

Bonehead sculpin.

Rosylip sculpin .

Spinynose sculpin .

Crested sculpin

Silverspotted sculpin

Roughback sculpin.

Sharpnose sculpin.

Wooly sculpin

Calico sculpin .

Mosshead sculpin .

Bald sculpin.

Coastrange sculpin

Eagle sculpin.

Prickly sculpin

Rough sculpin.

Mottled sculpin

Piute sculpin.

Malheur sculpin.

Banded sculpin .

Slimy sculpin

Shoshone sculpin

Riffle sculpin

Columbia sculpin

Marbled sculpin.

Wood River sculpin.

Margined sculpin .

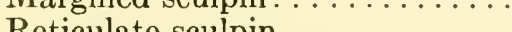

Klamath Lake sculpin .......

Torrent sculpin . . . . . . . . . .

Spoonhead sculpin.

Slender sculpin.

Spinyhead sculpin

Buffalo sculpin.

Bull sculpin.

Soft sculpin

Arctic staghorn sculpin

Red Irish lord.

Brown Irish lord

Sea raven.

Northern sculpin

Dusky sculpin .

Pit-head sculpin.

Threadfin sculpin

Fringed sculpin .

Frogmouth sculpin

Yellowchin sculpin.

Spotfin sculpin.

Twohorn sculpin

Spatulate sculpin

Longfin sculpin.
$\mathrm{P}$

$\mathrm{P}$

$\mathrm{P}$

$\mathrm{P}$

$\mathrm{P}$

$\mathrm{P}$

$\mathrm{P}$

$\mathrm{P}$

$\mathrm{P}$

$\mathrm{P}$

$\mathrm{P}$

$\mathrm{P}-\mathrm{F}$

$\mathrm{P}$

$\mathrm{P}$

$\mathrm{P}$

$\mathrm{P}$

$\mathrm{P}-\mathrm{F}$

$\mathrm{F}$

P-F

$\mathrm{F}$

F

F

$\mathrm{F}$

F

F

F

$\mathrm{F}$

F

$\mathrm{F}$

$\mathrm{F}$

F

F

$\mathrm{F}$

F

$\mathrm{F}$

$\mathrm{F}$

$\mathrm{P}$

$\mathrm{P}$

$\mathrm{P}$

$\mathrm{P}$

A-P

$\mathrm{P}$

$\mathrm{P}$

A

$\mathrm{P}$

$\mathrm{P}$

$\mathrm{P}$

$\mathrm{P}$

$\mathrm{P}$

$\mathrm{P}$

$\mathrm{P}$

$\mathrm{P}$

$\mathrm{A}$

A-P

$\mathrm{P}$
Artedius creaseri (Hubbs)

Artedius fenestralis Jordan and Gilbert Artedius hankinsoni (Hubbs) Artedius harringtoni (Starks)

Artedius lateralis (Girard)

Artedius notospilotus Girard

Ascelichthys rhodorus Jordan and Gilbert

Asemichthys taylori Gilbert

Blepsias bilobus Cuvier

Blepsias cirrhosus (Pallas)

Chitonotus pugetensis (Steindachner)

Clinocottus acuticeps (Gilbert)

Clinocottus analis (Girard)

Clinocottus embryum (Jordan and Starks)

Clinocottus globiceps (Girard)

Clinocottus recalvus (Greeley)

Cottus aleuticus Gilbert

Cottus annae Jordan and Starks

Cottus asper Richardson

.Cottus asperrimus Rutter

Cottus bairdi Girard

Cottus beldingi Eigenmann and Eigenmann

. Cottus bendirei (Bean)

Cottus carolinae (Gill)

Cottus cognatus Richardson

Cottus greenei (Gilbert and Culver)

Cottus gulosus (Girard)

Cottus hubbsi Bailey and Dimick

Cottus klamathensis Gilbert

Cottus lciopomus Gilbert and Evermann

Cottus marginatus (Bean)

. Cottus perplexus Gilbert and Evermann

Cottus princeps Gilbert

Cottus rhotheus (Smith)

Cottus ricei (Nelson)

Cottus tenuis (Evermann and Meek) Dasycottus setiger Bean

Enophrys bison (Girard)

Enophrys taurina Gilbert

Gilbertidia sigalutes (Jordan and Starks)

.Gymnocanthus tricuspis (Reinhardt)

Hemilepidotus hemilepidotus (Tilesius)

Hemilepidotus spinosus (Ayres)

Hemitripterus americanus (Gmelin)

Icelinus borealis Gilbert

Icelinus burchami Evermann and Goldsborough

Icelinus cavifrons Gilbert

Icelinus filamentosus Gilbert

. Icelinus fimbriatus Gilbert

Icelinus oculatus Gilbert

Icelinus quadriseriatus (Lockington)

. Icelinus tenuis Gilbert

Icelus bicornis (Reinhardt)

Icelus spatula Gilbert and Burke Jordania zonope Starks 
Lavender sculpin........... P

Pacific staghorn sculpin....... P-F

Blackfin sculpin........... P

Grubby.................. A

Longhorn sculpin........... A

Great sculpin........... P

Fourhorn sculpin............ A-P

Arctic sculpin............. A-P

Shorthorn sculpin.......... A-P

Sailfin sculpin............ P

Tidepool sculpin ............ P

Saddleback sculpin......... P P

Rosy sculpin............ P

Fluffy sculpin............ P

Snubnose sculpin........... P

Thornback sculpin.......... P

Tadpole sculpin............ . P

Slim sculpin............ P

Darter sculpin............ P

Smoothgum sculpin......... P

Grunt sculpin............ P

Puget Sound sculpin......... P

Cabezon............... P

Manacled sculpin.......... P

Roughspine sculpin.......... P

Mailed sculpin.............. . A

Ribbed sculpin........... A-P
Leiocottus hirundo Girard

Leptocottus armatus Girard Malacocottus kincaidi Gilbert and Thompson Myoxocephalus aeneus (Mitchill) Myoxocephalus octodecemspinosus (Mitchill) Myoxocephalus polyacanthocephalus (Pallas) Myoxocephalus quadricornis (Linnaeus) .Myoxocephalus scorpioides (Fabricius) .Myoxocephalus scorpius (Linnaeus) Nautichthys oculofasciatus (Girard) Oligocottus maculosus Girard Oligocottus rimensis (Greeley) . Oligocottus rubellio (Greeley) .Oligocottus snyderi Greeley Orthonopias triacis Starks and Mann Paricelinus hopliticus

Eigenmann and Eigenmann Psychrolutes paradoxus Günther . Radulinus asprellus Gilbert . Radulinus boleoides Gilbert . Radulinus vinculus Bolin Rhamphocottus richardsoni Günther Ruscarius meanyi Jordan and Starks Scorpaenichthys marmoratus (Ayres) Synchirus gilli Bean Triglops macellus (Bean) Triglops nybelini Jensen Triglops pingeli Reinhardt

\section{Agonidae-poachers and alligatorfishes}

Northern spearnose poacher.... .

Southern spearnose poacher.....

Sturgeon poacher.

Atlantic poacher .

Smooth alligatorfish

Aleutian alligatorfish .

Alligatorfish

Arctic alligatorfish

Gray starsnout.

Spinycheek starsnout.

Bigeye poacher.

Blackfin poacher.

Rockhead.

Fourhorn poacher

Bering poacher

Warty poacher.

Pygmy poacher

Tubenose poacher

Sawback poacher.

Longnose poacher

Beardless poacher

Pricklebreast poacher

Blacktip poacher.
Agonopsis emmelane (Jordan and Starks) Agonopsis sterletus (Gilbert)

Agonus acipenserinus Tilesius

Agonus decagonus Bloch and Schneider Anoplagonus inermis (Günther) A spidophoroides bartoni Gilbert

Aspidophoroides monopterygius (Bloch) . Aspidophoroides olriki Lütken

. Asterotheca alascana (Gilbert) Asterotheca infraspinata (Gilbert) Asterotheca pentacanthus (Gilbert) Bathyagonus nigripinnis Gilbert. . Bothragonus swani (Steindachner) Hypsagonus quadricornis (Cuvier) Occa dodecaedra (Tilesius) . Occa verrucosa (Lockington) Odontopyxis trispinosa Lockington Pallasina barbata (Steindachner) . Sarritor frenatus (Gilbert) Sarritor leptorhynchus (Gilbert) Stelgis vulsa (Jordan and Gilbert) Stellerina xyosterna (Jordan and Gilbert) Xeneretmus latifrons (Gilbert) 
Cutfin poacher........... P

Stripefin poacher........... P

Bluespotted poacher......... P
Xeneretmus leiops Gilbert Xeneretmus ritteri Gilbert Xeneretmus triacanthus (Gilbert)

Cyclopteridae-lumpfishes and snailfishes

Smooth lumpsucker.......... P P

Longfin snailfish . . . . . . . . . . A

Globefish................ P

Arctic lumpsucker........... A

Lumpfish ................. A

Leatherfin lumpsucker........ A-P

Pacific spiny lumpsucker....... P

Atlantic spiny lumpsucker..... . A

Newfoundland spiny lumpsucker. A

Seasnail................ A

Spotted snailfish ........... P

Ribbon snailfish............ P

Polka-dot snailfish . . . . . . . . A A-P

Marbled snailfish............. P

Tidepool snailfish . . . . . . . . P P

Slipskin snailfish............ P

Gelatinous seasnail. ........... A-P

Striped seasnail............ A

Slimy snailfish............ P

Showy snailfish........... P

Ringtail snailfish........... P

Greenland seasnail.......... A

Tadpole snailfish............ P

Prickly snailfish............. P

Bering snailfish ........... P

Lobefin snailfish . . . . . . . . P
A ptocyclus ventricosus (Pallas)

Careproctus longipinnis Burke Cyclopterichthys glaber Steindachner Cyclopteropsis macalpini (Fowler) Cyclopterus lumpus Linnaeus

. Eumicrotremus derjugini Popov Eumicrotrcmus orbis (Günther) Eumicrotremus spinosus (Müller) Eumicrotremus terraenovae Myers and Böhlke Liparis atlanticus (Jordan and Evermann) Liparis callyodon (Pallas) Liparis cyclopus Günther Liparis cyclostigma Gilbert Liparis dennyi Jordan and Starks Liparis florac (Jordan and Starks) Liparis fucensis Gilbert .Liparis koefoedi Parr Liparis liparis (Linnaeus) Liparis mucosus Ayres Liparis pulchellus Ayres Liparis rutteri (Gilbert and Snyder) Liparis tunicatus Reinhardt ........ Nectoliparis pelagicus Gilbert and Burke ....................... Paraliparis deani Burke ........ Polypera beringiana (Gilbert and Burke) ...........Polypera greeni (Jordan and Starks)

Dactylopteridae-flying gurnards

Flying gurnard A Dactylopterus volitans (Linnaeus)

Trichodontidae-sandfishes

Sailfin sandfish........... P . . . . . . Arctoscopus japonicus (Steindachner) Pacific sandfish........... P . P......... Trichodon trichodon (Tilesius)

Ammodytidae-sand lances

American sand lance.......... A

Northern sand lance.......... A

Pacific sand lance.

Ammodytes americanus DeKay Ammodytes dubius Reinhardt . A mmodytes hexapterus Pallas

Opisthognathidae-jawfishes

Swordtail jawfish.

Yellowhead jawfish

Banded jawfish

Longtail jawfish

Spotfin jawfish.

Mottled jawfish.
A

A

Lonchopisthus lindneri Ginsburg

A

A

A

A
Opisthognathus aurifrons

(Jordan and Thompson) Opisthognathus fasciatus Longley athus lonchurus Jordan and Gilbert Opisthognathus macrognathus Poey .Opisthognathus maxillosus Poey 
Pensacola jawfish

Dusky jawfish.
A

A
Opisthognathus mystacinus (Jordan) .......... Opisthognathus whitehursti (Longley)

Bathymasteridae-ronquils

Alaskan ronquil............ P ...Bathymaster caeruleofasciatus Gilbert and Burke

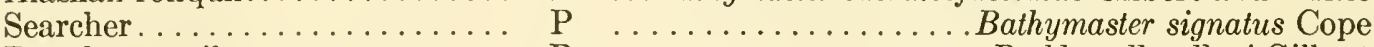
Rough ronquil........... $\mathrm{P} \ldots \ldots \ldots \ldots$ Rathbunella alleni Gilbert Smooth ronquil. ........... P $\quad \ldots \ldots \ldots \ldots$ Rathbunella hypoplectus (Gilbert)

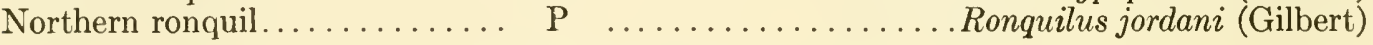

Percophididae-flatheads

Duckbill flathead

A Bembrops anatirostris Ginsburg Goby flathead.

A Bembrops gobioides (Goode)

Uranoscopidae-stargazers

Freckled stargazer........... A

Northern stargazer. . . . . . . . A

Southern stargazer........... A

A .....Arioscopus egregius (Jordan and Thompson)

Lancer stargazer............ A

................ Astroscopus guttatus Abbott

Smooth stargazer. Astroscopus y-graecum (Cuvier)

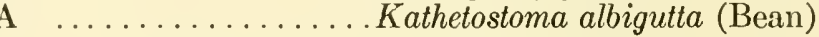
P .....Kathetostoma averruncus Jordan and Bollman

Dactyloscopidae-sand stargazers

Sand stargazer.

Arrow stargazer

Saddle stargazer
A

A

A
Dactyloscopus tridigitatus Gill Gillellus greyae Kanazawa A

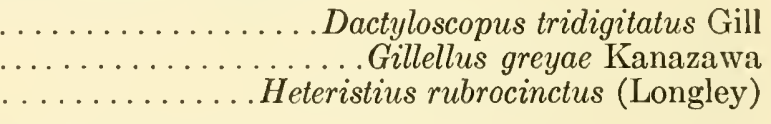

Callionymidae - dragonets

Lancer dragonet. . . . . . . . . A A

Coral dragonet.............. A

Spotted dragonet........... A
Callionymus bairdi Jordan Callionymus boekei Metzlaar

A .Callionymus calliurus Eigenmann and Eigenmann

\section{Clinidae - clinids}

Roughhead blenny........... A

Spinyhead blenny........... A

Island kelpfish............. P

Checkered blenny........... A

Orangethroat pikeblenny...... P

Bluethroat pikeblenny........ A

Deepwater blenny........... P

Banner blenny............. A

Glass blenny.............. A

Sailfin blenny............. A

Pirate blenny.............. A

Redeye blenny............. A

Spotted kelpfish............. P

Scarlet kelpfish............ P P

Striped kelpfish............ P

Crevice kelpfish........... P

Wrasse blenny............. A

Giant kelpfish . . . . . . . . . . . P
Acanthemblemaria aspera (Longley) Acanthemblemaria spinosa Metzelaar . Alloclinus holderi (Lauderbach)

Brannerella ocellata (Steindachner) Chaenopsis alepidota (Gilbert) .Chaenopsis ocellata Poey Cryptotrema corallinum Gilbert .............. Emblemaria diaphana (Longley) .....Emblemaria pandionis Evermann and Marsh .........Emblemaria piratula Ginsburg and Reid Enneapterygius pectoralis Fowler Gibbonsia elegans (Cooper) Gibbonsia erythra Hubbs Gibbonsia metzi Hubbs Gibbonsia montereyensis Hubbs Hemiemblemaria simulus Longley and Hildebrand Heterostichus rostratus Girard .... Emblemaria atlantica Jordan and Evermann 
Mimic blenny............. A

Downy blenny.............. A

Spotcheek blenny.......... A

Hairy blenny ............. A

Rosy blenny.............. A

Saddled blenny............ A

Sarcastic fringehead .......... P

Yellowfin fringehead.......... P

Onespot fringehead........... P

Coral blenny ............. A

Banded blenny ............ A

Horned blenny............. A

Reef finspot............ P

Marbled blenny............ A

Blackfin blenny............ A

Blackbelly blenny........... A

Eelgrass blenny........... A
.Labrisomus guppyi (Norman)

.Labrisomus kalisherae (Jordan) Labrisomus nigricinctus Howell Rivero Labrisomus nuchipinnis (Quoy and Gaimard) Malacoctenus macropus (Poey) Malacoctenus triangulatus Springer . Neoclinus blanchardi Girard Neoclinus stephensae Hubbs . Neoclinus uninotatus Hubbs Paraclinus cingulatus (Evermann and Marsh) .Paraclinus fasciatus (Steindachner) .Paraclinus grandicomis (Rosén) . Paraclinus integripinnis (Smith) Paraclinus marmoratus (Steindachner) Paraclinus nigripinnis (Steindachner) . Stathmonotus hemphilli Bean Stathmonotus stahli (Evermann and Marsh)

\section{Blenniidae-combtooth blennies}

Molly miller............. A

Seaweed blenny............ A

Highfin blenny............ A

Fringe blenny........... A

Striped blenny............. A

Florida blenny............. A

Pearl blenny............. A

Barred blenny............ A

Crested blenny. ........... A

Bay blenny.............. P

Rockpool blenny............ P

Feather blenny............ A

Freckled blenny............. A

Redlip blenny............ A
Blennius cristatus Linnaeus Blennius marmoreus Poey Blennius nicholsi Tavolga Blennius pilicornis Cuvier Chasmodes bosquianus (Lacépède) Chasmodes saburrae Jordan and Gilbert Entomacrodus textilis (Quoy and Gaimard) Hypleurochilus bermudensis Beebe and Tee-Van Hypleurochilus geminatus (Wood) Hypsoblennius gentilis (Girard) Hypsablennius gilberti (Jordan) Hypsoblennius hentzi (LeSueur) . . . . Hypsoblennius ionthas (Jordan and Gilbert) ......... Ophioblennius atlanticus (Valenciennes)

Anarhichadidae-wolffishes

Northern wolffish

Atlantic wolffish .

Spotted wolffish

Bering wolffish

Wolf-eel.

Penpoint gunnel

Stippled gunnel

Banded gunnel.

Bering gunnel.

Rock gunnel. .

Crescent gunnel.

Saddleback gunnel.

Red gunnel.

Kelp gunnel.

Rockweed gunnel

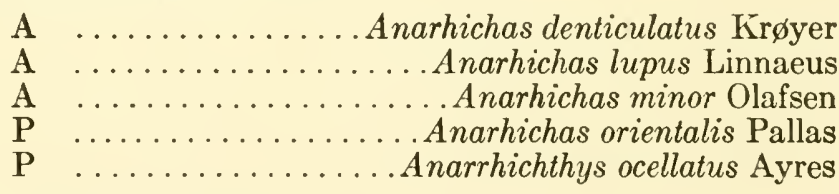

Pholidae-gunnels

$\mathrm{P}$ $\mathrm{P}$

A

$\mathrm{P}$

A

$\mathrm{P}$

$\mathrm{P}$

$\mathrm{P}$

$\mathrm{P}$

$\mathrm{P}$
Apodichthys flavidus Girard

Pholis fasciata (Bloch and Schneider)

Pholis gilli Evermann and Goldsborough Pholis gunnellus (Linnaeus) Pholis laeta (Cope) Pholis ornata (Girard) Pholis schultzi Hubbs ....... . Ulvicola sanctaerosae Gilbert and Starks Xererpes fucorum (Jordan and Gilbert)
Pholis dolichogaster (Pallas) 


\section{Stichaeidae ${ }^{1}$-pricklebacks}

Y-blenny.................. P ...Allolumpenus hypochromus Hubbs and Schultz

Cockscomb................. P . . ............Anoplarchus purpurescens Gill

Monkeyface blenny........... P .............Cebidichthys violaceus (Girard)

Mosshead prickleback........ P . . ... Chirolophus nugator (Jordan and Williams)

Decorated blenny........... P P . . . . . . Chirolophus polyactocephalus (Pallas)

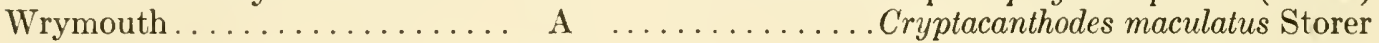

Giant wrymouth........... $\mathrm{P} \quad \ldots \ldots \ldots \ldots \ldots \ldots$ Delolepis gigantea Kittlitz

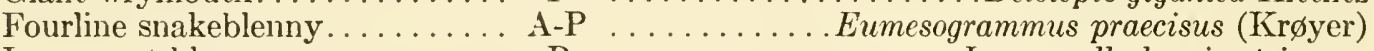

Longsnout blenny.......... $\mathrm{P} \quad \ldots \ldots \ldots \ldots \ldots \ldots$. Lumpenella longirostris

(Evermann and Goldsborough)

Slender eelblenny........... A-P Lumpenus fabricii (Valenciennes)

Snake blenny.............. A

Shanny ................. A-P

Stout eelblenny............ A-P

Pacific snakeblenny......... $\mathrm{P}$

Dwarf wrymouth........... P

Ribbon prickleback.......... P

Crisscross prickleback........ P

Bluebarred prickleback........ P

Whitebarred blenny......... P

Arctic shanny ............. A-P

Radiated shanny............ A

Black prickleback.......... P

Rock blenny.............. P ...................... Lumpenus maculatus (Fries) P .................Lumpenus medius Reinhardt ................ Lumpenus sagitta Wilimovsky ...................... ........Phytichthys chirus (Jordan and Gilbert) ............. Plagiogrammus hopkinsi Bean ................ Plectobranchus evides Gilbert . . . Poroclinus rothrocki Bean Stichaeus punctatus (Fabricius)

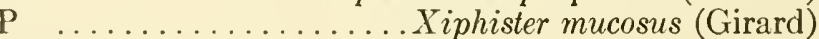

Ptilichthyidae-quillfishes

Quillfish............... P Ptilichthys goodei Bean

Zoarcidae ${ }^{2}$-eelpouts

Bigfin eelpout............. P

Snakehead eelpout........... P

Fish doctor.............. A

Wolf eelpout............ A

Blackmouth eelpout..........

Pallid eelpout...............

Shortfin eelpout.............

Black eelpout..............

Newfoundland eelpout..........

Wattled eelpout............... P

Pale eelpout............. A

Arctic eelpout.............. A

Polar eelpout............. A-P

Blackbelly eelpout......... P

Bearded eelpout........... P

Ocean pout.............. A

Aprodon cortezianus Gilbert Embryx crotalina (Gilbert)

Gymnelis viridis (Fabricius)

A $\ldots \ldots \ldots$ Lycenchelys verrilli (Goode and Bean)

P ................... Lycodapus fierasfer Gilbert

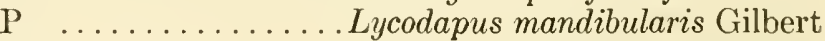

P ................................ brevipes Bean

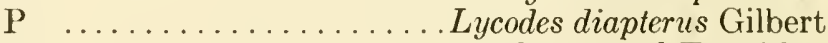

A ......... Lycodes lavalaei Vladykov and Tremblay

P ......................Lycodes palearis Gilbert

A $\ldots \ldots \ldots \ldots \ldots \ldots$. Lycodes pallidus Collett

A .................. Lycodes reticulatus Reinhardt

................ Lycodopsis pacifica (Collett)

A ...Macrozoarces americanus (Bloch and Schneider)

Scytalinidae-graveldivers

Graveldiver.

\footnotetext{
1 In addition to those listed, at least six species occur in our area only in Bering Sea. Too little is known about these to permit proposal of common names at this time.

${ }_{2}$ In addition to species listed, there are otbers that live in deep water off our coasts and some that occur over the continental shelf in northern seas, especially around Alaska.
} 


\section{Zaproridae-prowfishes}

Prowfish. $\mathrm{P}$ Zaprora silenus Jordan

Brotulidae - brotulas

Red brotula .

A ..........Brotula barbata (Bloch and Schneider)

Bearded brotula

A

Brosmophycis marginata (Ayres)

Kiey brotula.

Ophidiidae-cusk-cels

Fawn cusk-eel

Blackedge cusk-eel

Mottled cusk-eel.

Bank cusk-eel . . .

Pencil cusk-eel

Mooneye cusk-eel

Crested cusk-eel.

Polka-dot cusk-eel

Basketweave cusk-eel.......... .

Spotted cusk-eel.

Striped cusk-eel.
A

A

A

A

$\mathrm{P}$

$\mathrm{A}$

A

A

$\mathrm{P}$

$\mathrm{P}$

A
Lepophidium cervinum (Goode and Bean)

Lepophidium graellsi (Poey)

Lepophidium jeannae Fowler

Ophidion holbrooki (Putnam)

Ophidion novaculum Harry

Ophidion selenops Robins and Böhlke

Ophidion welshi (Nichols and Breder)

Otophidium omostigmum (Jordan and Gilbert)

Otophidium scrippsae Hubbs

. Otophidium taylori (Girard)

. Rissola marginata (DeKay)

Carapidae-pearlfishes

Pearlfish

Carapus bermudensis (Jones)

Stromateidae-butterfishes

Black ruff.

Longfin cigarfish

Silver-rag.

Medusafish................................

Blackrash..................

Man-of-war fish $\ldots \ldots \ldots \ldots$

Barrelfish...

Pacific pompano..............

Southern harvestfish..........

Northern harvestfish

Butterfish.

Freckled driftfish

Silver driftfish

Spotted driftfish
A

$\mathrm{P}$

A

$\mathrm{P}$

$\mathrm{P}$

A

A

$\mathrm{P}$

A

A

A

A

A

A
Centrolophus niger (Gmelin) Cubiceps gracilis (Lowe) Cubiceps nigriargenteus Ginsburg Icichthys lockingtoni Jordan and Gilbert Icticus pellucidus (Lütken) . Nomeus gronowi (Gmelin) Palinurichthys perciformis (Mitchill) Palometa simillima (Ayres) Peprilus alepidotus (Linnaeus) ... Peprilus paru (Linnaeus) Poronotus triacanthus (Peck) Psenes cyanophrys Cuvier Psenes maculatus Lütken .Psenes regulus Poey

Tetragonuridae-squaretails

Bigeye squaretail............ A

Smalleye squaretail.......... P

Tetragonurus atlanticus Lowe P ..................Tetragonurus cuvieri Risso

Icosteidae-ragfishes

Ragfish .

Pacific barracuda............ P

Great barracuda ............. A

Northern sennet.

A Sphyraena argentea Girard Sphyraena barracuda (Walbaum) Sphyraena borealis DeKay 
Guaguanche.............. A

Southern sennet

A
Sphyraena guachancho Cuvier Sphyraena picudilla Poey

Mugilidae-mullets

Mountain mullet........... A-F

Striped mullet ${ }^{1}$

White mullet. .

Redeye mullet

Liza.

Fantail mullet
A-F-P

A A A A
Agonostomus monticola (Bancroft) .Mugil cephalus Linnaeus Mugil curema Valenciennes Mugil gaimardiana Desmarest ...... Mugil liza Valenciennes Mugil trichodon Poey

\section{Atherinidae - silversides}

Reef silverside............. A

Hardhead silverside......... A

Topsmelt............... P-F

Jacksmelt................ . P

Brook silverside........... F

California grunion........... P

Rough silverside........... A

Mississippi silverside......... F

Tidewater silverside......... A-F

Key silverside........... A

Waccamaw silverside.......... F

Atlantic silverside.......... A

Allanctta harringtonensis (Goode) Atherinomorus stipes (Müller and Troschel) Atherinops affinis (Ayres) Atherinopsis californiensis Girard Labidesthes sicculus (Cope) Leuresthes tenuis (Ayres) Membras martinica (Valenciennes) Menidia audens Hay Menidia beryllina (Cope) A...Menidia conchorum Hildebrand and Ginsburg F...........Menidia extensa Hubbs and Raney A $\quad . . . . . . . . . . . M e n i d i a$ menidia (Linnaeus)

Polynemidae - threadfins

Pacific threadfin ........... P

Atlantic threadfin............ A

Yellow threadfin............ P

Barbu .
P ....Polydactylus approximans (Lay and Bennett) Polydactylus octonemus (Girard) Polydactylus opercularis (Gill) A ............. Polydactylus virginicus (Linnaeus)

\section{Order Pleuronectiformes (Heterosomata)}

Bothidae-lefteye flounders

Three-eye flounder.......... A

Ocellated flounder........... A

Peacock flounder............. A

Eyed flounder............ A

Pelican flounder............ A

Gulf Stream flounder......... A

Horned whiff............. A

Spotted whiff............. A

Pacific sanddab............ P

Bay whiff ................ A

Speckled sanddab............. $\quad \mathrm{P}$

Longfin sanddab.............. P

Mexican flounder........... A

Spotfin flounder............. A

Spiny flounder............. A

Fringed flounder............ $\Lambda$

Smallmouth flounder......... A
Ancylopsetta dilecta (Goode and Bean) . Ancylopsetta quadrocellata Gill Bothus lunatus (Linnaeus) Bothus ocellatus (Agassiz) .Chascanopsetta lugubris Alcock Citharichthys arctifrons Goode Citharichthys cornutus (Günther) Citharichthys macrops Dresel Citharichthys sordidus (Girard) Citharichthys spilopterus Günther ...... Citharichthys stigmaeus Jordan and Gilbert ............. Citharichthys xanthostigma Gilbert Cyclopsetta chittendeni Bean Cyclopsetta fimbriata (Goode and Bean) Engyophrys sentus Ginsburg Etropus crossotus Jordan and Gilbert Etropus microstomus (Gill)

1 Known as black mullet in Florida. 
Gray flounder

Shrimp flounder

Bigmouth sole.

Slim flounder.

Deepwater flounder.

Gulf flounder.

California halibut.

Summer flounder.

Southern flounder.

Fourspot flounder.

Broad flounder.

Windowpane.

Shoal flounder. .

Channel flounder .

Dusky flounder

Sash flounder.

Fantail sole.
A

A

$\mathrm{P}$

A

A

A

$\mathrm{P}$

A

A

A

A

A

A

A

A

A

$\mathrm{P}$
Etropus rimosus Goode and Bean Gastropsetta frontalis Bean

.Hippoglossina stomata Eigenmann and Eigenmann . Monolene antillarum Norman . Monolene sessilicauda Goode Paralichthys albigutta Jordan and Gilbert .Paralichthys californicus (Ayres) Paralichthys dentatus (Linnaeus) Paralichthys lethostigma Jordan and Gilbert Paralichthys oblongus (Mitchill) Paralichthys squamilentus Jordan and Gilbert Scophthalmus aquosus (Mitchill) Syacium gunteri Ginsburg Syacium micrurum Ranzani Syacium papillosum (Linnaeus) Trichopsetta ventralis (Goode and Bean) .Xystreurys liolepis Jordan and Gilbert

Pleuronectidae - righteye flounders

Arrowtooth flounder.......... P

Deepsea sole.............. P

Petrale sole............... P

Witch flounder............. A

Rex sole............... P

Flathead sole............. P

American plaice............. A

Bering flounder............. P

Atlantic halibut............. A

Pacific halibut............ P

Diamond turbot.......... P

Butter sole................. P

Rock sole............... P

Yellowfin sole............ P

Yellowtail flounder........... A

Longhead dab............. P

Arctic flounder . . . . . . . . . . . P

Smooth flounder........... A

Slender sole.............. P P

Dover sole................ P

English sole............. P

Starry flounder. . . . . . . . . . P-F

Alaska plaice............. P

C-O sole................ P P

Curlfin sole............... P

Spotted turbot............. P

Hornyhead turbot........... P

Sand sole. . ............. P

Winter flounder............ A

Greenland halibut.......... A
Atheresthes stomias (Jordan and Gilbert) Embassichthys bathybius (Gilbert) . Eopsetta jordani (Lockington) Glyptocephalus cynoglossus (Linnaeus) Glyptocephalus zachirus Lockington .... Hippoglossoides elassodon Jordan and Gilbert .Hippoglossoides platessoides (Fabricius) Hippoglossoides robustus Gill and Townsend ..................... Hippoglossus stenolepis Schmidt Hypsopsetta guttulata (Girard) Isopsetta isolepis (Lockington) Lepidopsetta bilineata (Ayres) Limanda aspera (Pallas) Limanda ferruginea (Storer) Limanda proboscidea Gilbert Liopsetta glacialis (Pallas) Liopsetta putnami (Gill) Lyopsetta exilis (Jordan and Gilbert) Microstomus pacificus (Lockington) Parophrys vetulus Girard Platichthys stellatus (Pallas) Pleuronectes quadrituberculatus Pallas .Pleuronichthys coenosus Girard ......Pleuronichthys decurrens Jordan and Gilbert ........Pleuronichthys ritteri Starks and Morris ......Pleuronichthys verticalis Jordan and Gilbert .............Psettichthys melanostictus Girard ........Pseudopleuronectes americanus (Walbaum) ........Reinhardtius hippoglossoides (Walbaum)

Soleidae-soles

A

Naked sole.

Scrawled sole............. A

Hogchoker. A

....................... Achirus lineatus (Linnaeus) Gymnachirus nudus Kaup Trinectes inscriptus (Gosse) A

A




\section{Cynoglossidae - tonguefishes}

California tonguefish........ . P ......Symphurus atricauda (Jordan and Gilbert)

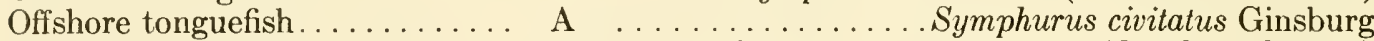

Spottedfin tonguefish......... A ......Symphurus diomedianus (Goode and Bean)

Largescale tonguefish........ A ............... Symphurus minor Ginsburg

Pygmy tonguefish.......... A .............. Symphurus parvus Ginsburg

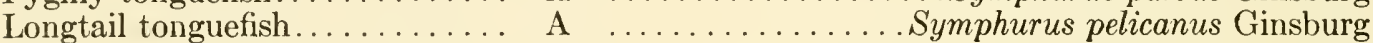

Deepwater tonguefish........ A ..........Symphurus piger (Goode and Bean)

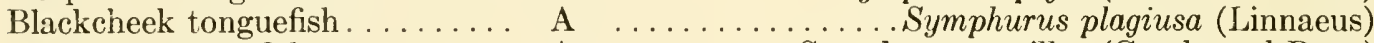

Northern tonguefish......... A .........Symphurus pusillus (Goode and Bean)

Spottail tonguefish......... A ............ Symphurus urospilus Ginsburg

Order Echeneiformes (Discocephali)

Echeneidae-remoras

Sharksucker.............. A

Whitefin sharksucker........ A

Slender suckerfish........... A

Whalesucker............... A-P

Spearfish remora........... A-P

Marlinsucker... . . . . . . . . . A-P

Remora................. A-P

White suckerfish . . . . . . . . . A-P

Echeneis naucrates Linnaeus Echeneis neucratoides Zuieuw Phtheirichthys lineatus (Menzies) ...................... Remora australis (Bennett) ............... Remora brachyptera (Lowe) A-P .... Remorina albescens (Temminck and Schlegel)

\section{Order Gobiesociformes (Xenopterygit)}

\section{Gobiesocidae-clingfishes}

Emerald clingfish.......... A ...Acyrtops beryllinus (Hildebrand and Ginsburg)

Northern clingfish .......... P . . . . . . . . . Gobiesox maeandricus (Girard)

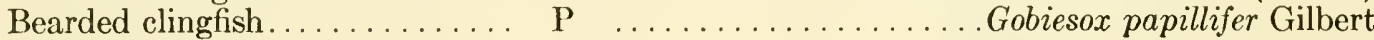

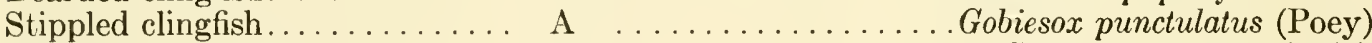

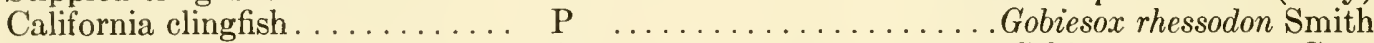

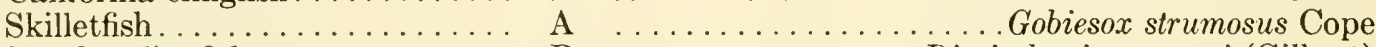

Slender clingfish .......... P . . . . . . . . . Rimicola eigenmanni (Gilbert)

Kelp clingfish............. P . . . Rimicola muscarum (Meek and Hildebrand)

\section{Order Tetraodontiformes (Plectognathi)}

Triacanthodidae-spikefishes

Jambeau

\section{Balistidae-triggerfishes and filefishes}

Unicorn filefish............ A

Orange filefish............ A

Scrawled filefish............ A

Dotterel filefish............ A

Gray triggerfish............. A

Spotted triggerfish .......... A

Finescale triggerfish ......... P

Queen triggerfish............ A

Orangespotted filefish......... A

Rough triggerfish........... A

Ocean triggerfish........... A

Black durgon.............. A
Alutera monoceros (Linnaeus) . Alutera schoepfi (Walbaum) Alutera scripta (Osbeck) Alutera ventralis Longley Balistes capriscus Gmelin Balistes forcipatus Gmelin Balistes polylepis Steindachner Balistes vetula Linnaeus Cantherines pullus (Ranzani) Canthidermis maculatus (Bloch) . Canthidermis sufflamen (Mitchill) . Melichthys radula (Solander) 
Fringed filefish

Planehead filefish

Pygmy filefish

Slender filefish

Redtail triggerfish

Sargassum triggerfish
A

A

A

A

$\mathrm{P}$

A
Monacanthus ciliatus (Mitchill)

Monacanthus hispidus (Linnaeus)

Monacanthus setifer Bennett Monacanthus tuckeri Bean Xanthichthys lineopunctatus (Hollard) .Xanthichthys ringens (Linnaeus)

Ostraciidae-trunkfishes

Spotted trunkfish

A

A

A

A $\mathrm{P}$
Lactophrys bicaudalis (Linnaeus) Lactophrys quadricornis (Linnaeus) Lactophrys trigonus (Linnaeus) Lactophrys triqueter (Linnaeus) Lactoria diaphana (Bloch and Schneider)

Trunkfish......

Spiny trunkfish

Tetraodontidae--puffers

Sharpnose puffer

Smooth puffer
A

A

$\mathrm{P}$

A

$\mathrm{P}$

A

A

A

A

A
Canthigaster rostrata (Bloch) Lagocephalus laevigatus (Linnaeus) Lagocephalus lagocephalus (Linnaeus) . Lagocephalus pachycephalus (Ranzani) Sphaeroides annulatus (Jenyns) Sphaeroides dorsalis Longley

Northern puffer

Southern puffer

Bandtail puffer.

Checkered puffer

\section{Diodontidae - porcupinefishes}

Pacific burrfish............ P

Bridled burrfish............. A

Web burrfish.

Spotted burrfish

Striped burrfish

Balloonfish.

$\mathrm{P}$

Chilomycterus affinis Günther

A

Chilomycterus antennatus (Cuvier)

A

A

$A$

Porcupinefish

santillarum Jordan and Rutter

Chilomycterus atinga (Linnaeus)

Chilomycterus schoepfi (Walbaum)

.Diodon holacanthus Linnaeus Diodon hystrix Linnaeus

Molidae-molas

Sharptail mola............. A

Mola lanceolata Lienard

Ocean sunfish

$\mathrm{P}$ Mola mola (Linnaeus)

Slender mola.

\section{Order Batrachoidiformes (Haplodoci)}

Batrachoididae - toadfishes

Gulf toadfish.............. A

Leopard toadfish............ A

Oyster toadfish............ A

Slim midshipman........... P

Northern midshipman........ $\mathrm{P}$

A .................. Opsanus beta (Goode and Bean)

Atlantic midshipman .

A
................. Opsanus pardus (Goode and Bean)

...................... Opsanus tau (Linnaeus)

.........Porichthys myriaster Hubbs and Schultz

....................... Porichthys notatus Girard ............. Porichthys porosissimus (Cuvier)

\section{Order Lophiformes (Pediculati)}

Lophiidae-goosefishes

Goosefish 
Antennariidae-frogfishes

Roughjaw frogfish.......... P ........Antennarius avalonis Jordan and Starks

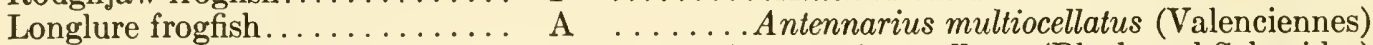

Ocellated frogfish............ A .....Antennarius ocellatus (Bloch and Schneider)

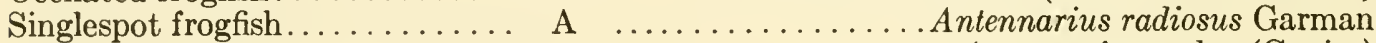

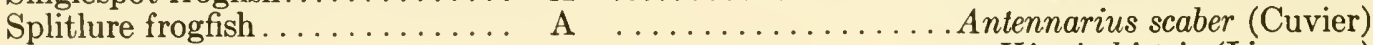

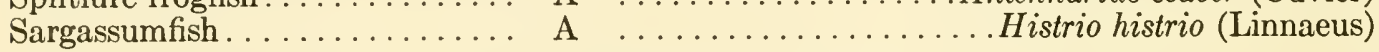

Ogcocephalidae-batfishes

Spiny batfish.

Tricorn batfish. .

Shortnose batfish

Roughback batfish

Polka-dot batfish

Longnose batfish

Spotted batfish
A ............Halieutichthys aculeatus (Mitchill) A ............... Ogcocephalus mcgintyi Fowler

A ........... Ogcocephalus nasutus (Valenciennes)

A ..... Ogcocephalus parvus Longley and Hildebrand

A ................ Ogcocephalus radiatus (Mitchill)

A $\quad . . \ldots \ldots$. Ogcocephalus vespertilio (Linnaeus)

P .................. (Joutes elater (Jordan and Gilbert) 


\section{PART II}

\section{Index to Common Names}

$\mathbf{A}$

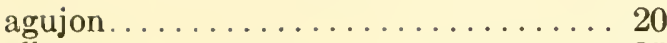

albacore................... 35

false-see tuna, little

alewife................... 10

alligatorfish................ 40

Aleutian. . . . . . . . . . . . . . . . 40

Aretic................... 40

smooth................. 40

alligatorfishes.............. 40

allmouth-see goosefish

amberjack, false-see amberjack, lesser greater..................... 30

lesser..................... 30

Pacific..................... 30

slender-see rudderfish, banded

anchoveta.................. 11

anchovies.................. 11

anchovy, bay................ 11

Cuban................... 11

deepbody.................. 11

dusky . . . . . . . . . . . . . . . 11

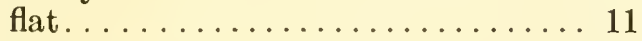

key.................... 11

longnose. . . . . . . . . . . . . 11

New Jersey.................... 11

northern................. 11

Pacific-see anchovy, northern

silver................... 11

$\operatorname{slim} \ldots \ldots \ldots \ldots \ldots \ldots \ldots \ldots 11$

slough................. 11

striped.................. 11

angelfish, black-see angelfish, French; angelfish, gray

blue

French.................... 32

gray ..................... 32

queen. . . . . . . . . . . . . . . . . 32

white-see spadefish, Atlantic

angler-see goosefish

argentine, Atlantic............... 12

Pacific.................... 12

argentines................ 12

\section{B}

bairdiella

balao........................ 20

balloonfish..................... 49

ballyhoo.................... 20

redtail-see ballyhoo barbfish.................. 36

barbier, red................ 25

barbu..................... 46

barracuda, California-see barracuda, Pacific

great................... 45

northern-see sennet, northern

Pacific..................... 45

barracudas................... 45

barreleye.................. 12

barreleyes.................... 12

barrelfish................. 45

bass, bank sea.............. 25

bigeye-see salema

black-see bass, largemouth; bass, smallmouth; bass, spotted; bass, redeye

blackear.

black sea................. 25

see also bass, giant sea

blue-see blacksmith; croaker, black; opaleye; sargo

bull-see bass, kelp

calico-see bass, kelp; crappie, black

chalk.

channel-see drum, red

Coosa-see bass, redeye

crimson.................. 25

giant sea............... 26

see also skilfish

Guadalupe................. 27

kelp.................. 25

Kentucky - see bass, spotted

lantern.................... 25

largemouth................ 27

longtail.................. 25

orangeback................ 25

pygmy sea................. 26

redeye................... 27

see also bass, rock

reef .................... 25

Roanoke................. 27

rock. .................... 27

rock sea.................. 25

roughtongue................ 25

saddle...................... 25

sand..................... 25

silver-see bass, white

smallmouth ................ 27

southern sea................ 25

speckled-see crappie, black 
bass, splittail............... 25

spotted.................. 27

spotted sand................ 25

stone-see wreckfish

strawberry-see crappie, black

streamer.................... 25

striped . . . . . . . . . . . . . . . 26

Suwannee.................. 27

white ...................... 26

yellow. . . . . . . . . . . . . . 26

yellowtail. . . . . . . . . . . . . 25

basses, sea. . . . . . . . . . . . 25

batfish, longnose . . . . . . . . . . . 50

polka-dot. . . . . . . . . . . . . 50

roughback................. 50

shortnose................. 50

spiny .................. 50

spotted.................. 50

tricorn. . . . ............. 50

baysmelt- see topsmelt

beadeye-see stonecat

beardfish . . . . . . . . . . . . . . . 24

beardfishes.................. 24

beaugregory .................... 33

beauty, rock................. 32

bergall-see cunner

bigeye. . . . . . . . . . . . . 26

short. . . . . . . . . . . . . 26

bigeyes................ 26

billfish-see needlefish; saury; gar

billfishes.

bitterling

blackback-see flounder, winter

blackcod--see sablefish

blackfish - see bass, black sea; tautog

Alaska.................... 12

Sacramento . . . . . . . . . . . 16

blackrag. . . . . . . . . . . . . 45

blacksmith . . . . . . . . . . . . . 33

blanquillo-see whitefish, ocean

blennies, combtooth............ 43

blenny, banded.............. 43

banner................... 42

barred................... 43

bay

see prickleback, bluebarred

belted-see prickleback, ribbon

black-see prickleback, black

blackbelly

blackfin .

bracketed-see gunnel, crescent

burrowing-see graveldiver

checkered

coral.

crested.

see also cockscomb

decorated.

deepwater. downy.................. 43

eelgrass.................. 43

feather.................. 43

Florida................... 43

freckled. . . . . . . . . . . . . . 43

fringe.................. 43

fucus-see gunnel, rockweed

glass.................... 42

hairy ................... 43

highfin.................. 43

horned .................. 43

longsnout. . . . . . . . . . . . . . 44

marbled.................. 43

mimic.................. 43

monkeyface............... 44

ornamented-see prickleback, mosshead

pearl. .................. 43

pen-point-see gunnel, penpoint

pirate................... 42

redeye................... 42

redlip................... 43

rock..................... 44

see prickleback, black

rockpool.................44

$\operatorname{rosy} \ldots \ldots \ldots \ldots \ldots \ldots \ldots \ldots \ldots \ldots$

roughhead ............... 42

saddled.................... 43

see gunnel, saddleback

sailfin ................... 42

seaweed ................. 43

snake.................. 44

spinyhead . . . . . . . . . . . . . 42

spotcheek................ 43

striped . . . . . . . . . . . . . . 43

whitebarred................ 44

wrasse................. 42

blindcat, toothless.............. 19

widemouth ................. 19

blindfish - see cavefish

bloater................... 11

bluefish.................... 29

bluefishes. . . . . . . . . . . . . . 29 29

bluegill . . . . . . . . . . . . . . . 27

bluehead. . . . . . . . . . . . . . 34

boarfish, deepbody . . . . . . . . . 24 shortspine................. 24

boarfishes................. 24

43 bobo-see threadfin, Pacific

43 bocaccio................... 37

bonefish.................. 10

bonefishes................. 10

bonehead-see bonito, Pacific

bonito, Atlantic............... 35

California-see bonito, Pacific

oceanic-see tuna, skipjack

Pacific .

striped................. 35 
bonnethead

bony fishes.

bonytail.

boo hoo-see mackerel, frigate

bowfin.

bowfins.

boxfish-see trunkfish spiny-see burrfish, striped

bream - see pinfish; bluegill

Ray's-see pomfret

sea.

brill-see sole, petrale

broadbill-see swordfish

brotula, bearded .

key.

red.

brotulas.

buffalo, bigmouth

black

gourd-head-see buffalo, bigmouth

highback-see buffalo, smallmouth

mongrel-see buffalo, black

razorback-see buffalo, smallmouth

redmouth - see buffalo, bigmouth

round-see buffalo, black

smallmouth

bull, Mexican-see margate, black

bullfish-see cabezon

bullhead-see also cabezon; sculpin, $\mathrm{Pa}$ cific staghorn

black.

brown .

flat. .

yellow

bumper

burbot.

burrfish, bridled................. 49

Pacific.... . . . . . . . . . . . . . . 49

spotted.................... 49

striped................... 49

web........................ 49

butterfish

see also gunnel, rock

butterfishes

butterflyfish, banded

bank.

foureye.

longsnout

reef

spotfin

butterflyfishes

\section{C}

cabezon

see also sculpin, Pacific staghorn cabezone-see sculpin, Pacific staghorn cabio-see cobia

cabrilla, spotted.
8 candlefish-see eulachon

9 capelin

cardinalfish, barred.............. 26

belted..................... 26

bridle................... . 26

bronze................... 26

dusky . . . . . . . . . . . . . . . 26

freckled................... 26

pale.................... 26

sawcheek................. 26

twospot................. 26

whitestar. . . . . . . . . . . 26

cardinalfishes................. 26

carp..................... 13

carps.................... 13

carpsucker, highfin. . . . . . . . . . 17

plains................... 17

river................... 17

cartilaginous fishes............. 6

catalufa - see also bigeye

popeye................26

catfish, bigmouth - see catfish, gafftopsail

black - sce bullhead, black

blue.

brown-see bullhead, brown

channel...................... 18

flathead...................... 19

forktail-see catfish, white

gafftopsail. .

headwater.

mud-see catfish, flathead

Potomac-see catfish, white

sea. .

see also wolffish, Atlantic

shovelhead-see catfish, flathead

smallmouth-see catfish, sea

spoonbill-see paddlefish

squaretail-see bullhead, brown

white.

Yaqui.

yellow-see catfish, flathead

catfishes, freshwater............... 18

sea .................... 18

cavefish, northern............. 22

Ozark.................... 22

southern.................... . 22

spring.................... . 22

cavefishes.................... 22

cero...................... 35

chaetodon-see sunfish, blackbanded

char, Arctic..................... 12

Great Lakes - see trout, lake

speckled-see trout, brook

characins

charr-see char

cherubfish

chilipepper

chimaeras. 
chinafish-see filefish, scrawled; rockfish, china

chiro-see ladyfish

chiselmouth................ 13

chromis, blue................ 33

brown.................. 33

chub-see also cisco

Alabama . .................. 14

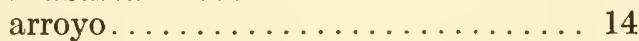

Bermuda.................. 32

bigeye....................... 14

blotched... . . . . . . . . . . . . . . 14

blue...................... 13

bluehead...................... 14

Columbia River-see peamouth

creek. . . . . . . . . . . . . . . . 17

flame..................... 14

flathead................... 14

gravel.................... 14

highback.................. 14

hornyhead. . . . . . . . . . . . . . . 14

humpback. ............... 13

Klamath-see chub, blue

lake. . . . . . . . . . . . . . . . . . 14

least. . . . . . . . . . . . . . . 14

leatherside.............. 17

Mohave........................ 17

northern-see chub, lake

Oregon.................... 14

peamouth - see peamouth

redeye................... 14

Rio Grande.................. 14

river. . . . . . . . . . . . . . . . . 14

rosyface................. 14

sicklefin.................... 14

silver. . . . . . . . . . . . . . . . . . . 14

slender.................... 14

Sonora..................... 14

speckled . . . . . . . . . . . . . . . . . 14

spotfin................... 14

streamline. . . . . . . . . . . . 14

sturgeon.................. 14

thicklip................. 14

thicktail. . . . . . . . . . . . . . 13

Tui...................... 17

Utah..................... 13

Yaqui........................ 14

yellow..................... . 32

chubs, sea.................... . 32

chubsucker, creek................ 17

lake..................... 17

sharpfin................. 17

cichlid, pearl-see perch, Rio Grande

velvet . .

cichlids.

cigarfish-see also scad, round; scad, bigeye

longfin. cisco...................... 11

Arctic................... 11

blackfin.................... 11

Bonneville.................... 11

deepwater.................... 11

least.................... 11

longjaw.................. 11

Nipigon..................... 11

shortjaw ................. 11

shortnose.................... 11

white-see cisco, deepwater

clingfish, bearded................ 48

California .................... 48

common-see clingfish, northern

emerald . . . . . . . . . . . . . . . 48

kelp................... 48

northern. . . . . . . . . . . . . . 48

slender. . . . . . . . . . . . . . . . . . 48

stippled.................... 48

clingfishes.................. 48

clinids................... . 42

cobbler-see mummichog

cobia. . . . . . . . . . . . . . . . . . 29

cobias. . . . . . . . . . . . . . . . 29

cockscomb. . . . . . . . . . . . . 44

cod, Arctic.................. 22

Atlantic.................... 22

black - see sablefish

blue-see cabezon

bull-see cabezon

channel-see rockfish, channel

chili-see chilipepper

cultus-see lingcod

Greenland.................... 22

northern-see cod, saffron

Pacific..................... 22

polar..................... 22

rock-see cod, Atlantic; rockfish

saffron..................... 22

codfishes.................... 22

combfish, longspine............. 38

shortspine................. 38

combfishes..................... 38

conchfish.................... 26

coney ....................... 25

conger, bandtooth. . . . . . . . . . . 19

Catalina................... 19

manytooth .................. 19

margintail................ 19

silver. . . . . . . . . . . . . . . . . 19

whiptail.................. 19

yellow. . . . . . . . . . . . . . . . 19

conny-see inconnu

convictfish-sce greenling, painted

corbina, California . . . . . . . . . . . . 31

cornetfish.................... 23

cornetfishes.................... 23

corvina, orangemouth............ 31 
corvina, shortfin............... 31

cottonwick.................. 31

cowfish....................... 49

crabeater-sce cobia

crampfish-see ray, lesser electric

crappie, black.

white.

creole-fish................... 25

crestfish.................. . 24

crestfishes................... 24

crevalle, green-see runner, blue

jack-see jack, crevalle

croaker, Atlantic.

black.

blue.

Catalina.

china-see croaker, black; sargo

golden-see croaker, spotfin

king-see croaker, white

reef...................... 31

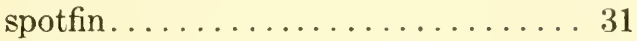

white.................. . 31

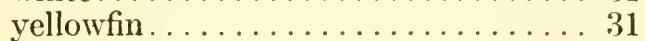

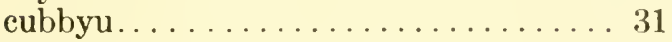

cui-ui. . . . . . . . . . . . . . . . . . 17

cultus, Pacific-see lingeod

cunner. . . .................. 34

cusk...................... 22

cusk-cel, bank. . . . . . . . . . . 45

basketweave............... 45

blackedge.................. 45

crested.................. 45

fawn ................... 45

mooneye.................. 45

mottled................... 45

pencil..................... 45

polka-dot................ 45

spotted................... 45

striped.................. 45

cusk-eels. . . . . . . . . . . . . . . . 45

cutlassfish, Atlantic............ 34

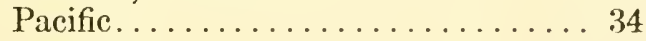

cutlassfishes................. 34

eyprinodon, variegated - see minnow, sheepshead

\section{D}

dab-see also sanddab

Atlantic sand-see windowpane

longhead.

mottled sand-see sanddab, Pacific

mud-see sole, yellowfin

rough-see plaice, American

rusty - see flounder, yellowtail

dace, blacknose

desert.

finescale.

horned-see chub, creek leopard................. 16

longfin................... 13

longnose................. 16

Moapa.................... 14

mountain redbelly............. 13

northern redbelly . . . . . . . . . . 13

pearl.................. 17

redside. . . . . . . . . . . . . . . 13

rosyside................... 13

southern redbelly. . . . . . . . . . 13

speckled . . . . . . . . . . . . . 16

Umpqua ................... 16

damselfish, bicolor. . . . . . . . . . . 33

cocoa...................... . 33

dusky .................. 33

threespot................ 33

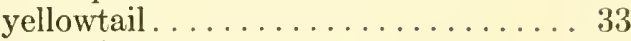

damselfishes................. 33

darter, Arkansas. . . . . . . . . . . . . 27

Arkansas saddled.............. 28

arrow.................... 28

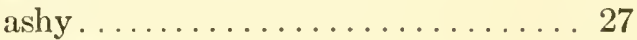

backwater................. 29

banded................... 28

barcheek. . . . . . . . . . . . . . . 28

blackbanded................ . 29

blackside................... 29

blackside snubnose............ . 27

blenny................... 27

bluebreast................. 27

blueside.................. 28

bluestripe................. 29

bluntnose.................. . 27

bronze................... 29

brown.................... 28

Carolina................... 27

channel..................... 29

Coosa..................... 27

crystal.................. 27

Cumberland snubnose.......... 27

cypress.................. 28

dusky . . . . . . . . . . . . . . 29

eastern sand .............. 27

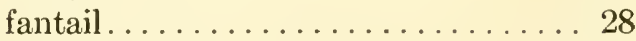

finescale saddled. . . . . . . . . . . 28

fountain . . . . . . . . . . . . 28

gilt. . . . . . . . . . . . . . 29

glassy . . . . . . . . . . . . . . 28

goldstripe. . . . . . . . . . . . 28

greenbreast................. 28

greenside.................. 27

greenthroat................ 28

gulf . . . . . . . . . . . . . 28

Harlequin ................. 28

Iowa. . . . . . . . . . . . . . . 28

Johnny. . . . . . . . . . . . . . . 28

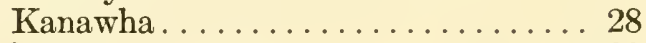

least..................... 28 
darter, leopard.

longfin.

longhead

longnose

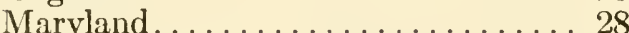

Missouri saddled............... 28

$\operatorname{mud} \ldots \ldots \ldots \ldots \ldots \ldots \ldots \ldots 27$

naked sand ................. 27

Niangua................... 28

olive..................... 29

orangebelly................. 28

orangethroat............... 28

Piedmont................... 29

pinewoods................. 28

rainbow................. 27

redband..................... 28

redfin..................... 28

redline.................. 28

Rio Grande................. 28

river..................... 29

riverweed ................. 28

rock.................... 28

Saluda..................... 28

Savannah................ 28

sawcheek.................. 28

scalyhead................. 27

scaly sand ................. 27

seagreen.................. 28

sharphead.................. 27

sharpnose................. 29

shield.......................... 29

slenderhead................... 29

slough................... 28

speckled................... 28

spottail.................. 28

spotted.................... 28

stargazing.................. 29

stippled..................... 28

stripeback................ 29

striped........................ 28

stripetail................. 28

swamp.................. 28

Swannanoa.................. 28

Tennessee snubnose. . . . . . . . . . . 28

Tippecanoe.................. 28

Tuckasegee................ 28

turquoise.................. 28

Tuscumbia................ 28

variegate................. 28

Waccamaw.................. 28

western sand . . . . . . . . . . . . . 27

yellow.................... 29

yoke..................... 28

dealfish..................... 24

devil, red-see wrymouth, dwarf

devilfish, little-see ray, devil

dick, slippery...

diver, sand. doctor, fish.................. 44

doctorfish .................. 34

dogfish, black............... 8

chain.................... 7

Cuban..................... 8

freshwater-see bowfin

smooth.................. 7

spiny.................... 8

dollarfish-see harvestfish

Dolly Varden.................... 12

dolphin..................... 30

pompano................... 30

dolphins.................... 30

dorado-see dolphin

doré-see walleye

dories........................ 24

Dory, American John................ 24

doughbelly - see stoneroller

dragonet, coral................4 42

lancer...................... 42

spotted ................... 42

dragonets................... 42

driftfish, freckled............... 45

silver...................... 45

spotted................... 45

drum, banded ................ 31

black..................... 31

freshwater................. 31

red......................... 31

sand ...................... 31

sea-see drum, black

spotted.................... 31

star......................... 31

striped.................... 31

drums.................... 31

durgon, black................ 48

E

eel, American................. 19

blotched snake.................. 19

California worm . . . . . . . . . . . . . 19

conger...................... 19

congo-see wrymouth, giant

finless................... 20

goldspotted ................. 20

horsehair.................. 19

key worm................. 19

lamprey - see lamprey

monkeyface - see blenny, monkeyface mustard ........................ 20

Pacific snake................... 20

palespotted.................. 20

ridgefin.................... 19

rock-see gunnel, rock

sailfin..................... 19

sand-see lance, sand

sharptail................... 19

shorttail snake............... 19 
eel, shrimp.

silver-see cutlassfish

slender pike.

slime- see hagfish

snapper.

sooty

speckled worm

spiny .

spotted snake.

spotted spoon-nose

stippled spoon-nose

surf.

thread.

tiger snake

whip

yellow snake.

eelback-see flounder, smooth

eelblenny, slender

stout.

eelpout--see also pout, ocean; burbot

Arctic.

bearded.

bigfin.

black .

blackbelly

blackmouth

Newfoundland.

pale.

pallid.

pearly-see eelpout, blackmouth

polar

shortfin

snakehead

wattled.

wolf

eelpouts.

eels, conger.

freshwater.

mustard.

snake

snipe .

spiny

escolar.

eulachon

$\mathbf{F}$

fallfish

fatback-see menhaden

fathead-see sheephead, California

filefish, common-see filefisin, planehead

dotterel.

fringed.

lettered-see filefish, scrawled

longtail-see filefish, scrawled

orange.

orangespotted

planehead.

pygmy scrawled

slender.

unicorn .

filefishes

finspot, reef

fish, dovetail-see sergeant, night

flag, Spanish. see also rockfish, flag

flagfish

flamefish.

flasher-see tripletail

flatfish-see flounder, winter

flathead, duckbill. goby

flatheads.

flier $\ldots \ldots \ldots \ldots \ldots \ldots \ldots \ldots \ldots \ldots \ldots \ldots \ldots \ldots \ldots \ldots$

flounder, Arctic............... 47

arrowtooth.............. 47

Bering. . . . . . . . . . . . . 47

broad.................. 47

broadfin-see sole, rock

channel

curlfin-see sole, curlfin

deepwater.

diamond-see turbot, diamond

dusky. . .

English-see sole, English

eyed

fantail-see sole, fantail

flathead-see sole, flathead

fourspot.

fringe-see sole, sand

fringed.

Georges Bank-see flounder, winter gray.

gulf .

Gulf Stream.

hornyhead-see turbot, hornyhead

lemon-see sole, English

longfin-see sole, rex

longjaw - see flounder, arrowtooth

Mexican.

ocellated

peacock

pelican .

pointed-nose- see sole, English

rock-see sole, rock

roundnose-see sole, petrale

sash

sand-see flounder, gulf; sole, sand

scalyfin-see sole, butter

shoal.

shrimp

slender-see sole, slender

slim

slime-see sole, Dover

slippery-see sole, Dover

smallmouth 
flounder, smooth.

southern

spiny.

spotfin.

starry.

summer.

three-eye.

winter.

witch.

yellowtail

flounders, lefteye

righteye.

fluke, fourspot-see flounder, fourspot gulf-see flounder, gulf

southern-see flounder, southern

summer-see flounder, summer

flyingfish, Atlantic.............. 20

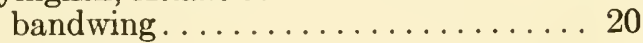

blackwing. ................ 21

bluntnose.................. 21

California................... 20

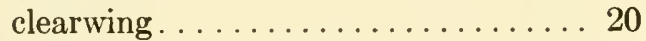

fourwing .................. 21

margined................. 20

oceanic two-wing............ 21

sailfin................... 21

sharpchin............... 21

smallwing ................ 21

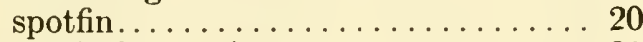

tropical two-wing . . . . . . . . . 21

flyingfishes ................. 20

foolfish-see filefish

fringehead, onespot............. 43

sarcastic .................. 43

yellowfin................ 43

frogfish, longlure. . . . . . . . . . . . 50

ocellated................ 50

roughjaw. . . . . . . . . . . . 50

singlespot................ 50

splitlure. . . . . . . . . . . . . . 50

frostfish-see tomcod, Atlantic; whitefish, round

\section{G}

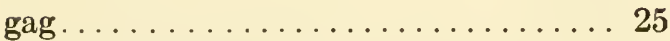

gambusia, Big Bend............ 22

blotched ..................... 22

Clear Creek. . . . . . . . . . . . . . 22

largespring . . . . . . . . . . . . 22

Pecos..................... 22

gar, alligator .................... 10

Florida.................. 10

longnose................. 10

saltwater-see needlefish

shortnose.................. 10

silver-see needlefish

spotted

garfish-see needlefish, California garibaldi.

garpike-see gar

gars.................... 10

gaspereau-see alewife

gaspergou - see drum, freshwater

ghostfish-see wrymouth

globefish.

see also sunfish, ocean

goatfish, dwarf............... 32

Mexican.................. 31

red....................... 31

spotted.................. 32

yellow.................. 31

goatfishes................. 31

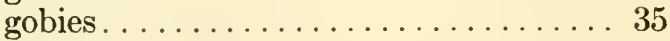

goby, arrow ....................... 35

banner...................... 36

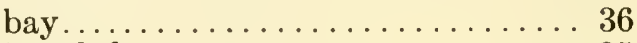

bearded.................... 35

blind .................. 36

bluebanded................. 36

bluespot. . . . . . . . . . . . . 35

bridled................... 35

cheekspot................ 36

clown................... 36

code............................... 36

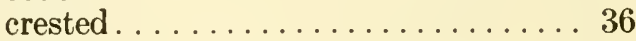

darter.................... 36

emerald . . . . ............ 36

finescale-see goby, bay

freshwater................ 36

frillfin................... 35

goldspot................. 36

green.................... 36

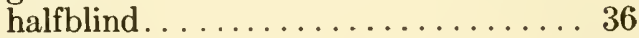

highfin................... 36

largescale-see goby, bluespot

longjaw-see mudsucker, longjaw

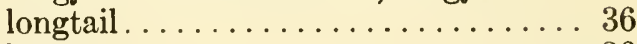

lyre . . . . . . . . . . . . . . . 36

marked ................. 36

naked.................... . 36

neon....................... 35

notchtongue ............... 35

orangespotted. . . . . . . . . . . 36

paleback.................. 36

river.................... 35

seaboard................ 36

Seminole.................. 36

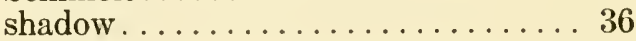

sharptail. . . . . . . . . . . . 36

$\operatorname{slim} \ldots \ldots \ldots \ldots \ldots \ldots \ldots \ldots \ldots$

smallmouth................. . 36

sponge.................. 36

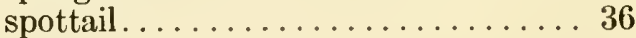

tidewater.................. 35

tiger.................... 36

twoscale................ 36 
goby, violet................... 36

white-eye. . . .............. 35

whiteline.................... 36

zebra.................. 36

goggle-eye-see bass, rock; scad, bigeye

goggler-see scad, bigeye

goldeye.

goldfish

Catalina - see garibaldi

ocean-see garibaldi

goody-see spot

goosefish.

goosefishes

goujon-see catfish, flathead

graveldiver.

graveldivers

grayback - see alewife

grayfish - see dogfish

grayling, Aretic.

Montana-see grayling, Arctic

graylings. ...

graysby

greenfish-see opaleye

greenling, Alaska-see greenling, masked common-see greenling, whitespotted fringed-see greenling, rock

kelp

longspine-see combfish, longspine

masked

painted

red-see greenling, rock

rock. . .

whitespotted.

greenlings .

grenadiers.

grindle-see bowfin

grouper, black .

broomtail. .

gulf

marbled

misty.

Nassau

red

rock-see bocaccio

salmon-see bocaccio

sicklefin - see grouper, marbled

snowy

tiger.

Warsaw.

yellowedge

yellowfin

yellowmouth

grubby

grunion, California . . . . . . .

grunt, barred..................... 30

black................... 30

blacktail-see grunt, Caesar

bluestriped. burro.

Caesar...................... 30

common-see grunt, white

French

redmouth-see tomtate

smallmouth. . ............... 30

Spanish..................... 31

white................... 31

yellow - see grunt, blucstriped; grunt, French

gruntfish-see sculpin, grunt

grunts........................ 30

guaguanche.................. 46

guitarfish, Atlantic............. 8

banded.................. 8

California - see guitarfish, shovelnose mottled—see guitarfish, banded

shovelnose................ 8

guitarfishes................... 8

gunnel, banded ................ 43

Bering................... 43

crescent.................. 43

kelp................... 43

penpoint................. 43

red........................ 43

rock...................... 43

rockweed.................... 43

saddleback................... 43

stippled................... 43

gunnels.................... 43

gurnard, flying............... 41

gurnards, flying.............. 41

\section{H}

hackleback-see sturgeon, shovelnose

haddock. . . . . . . . . . . . . . 22

hagfish, Atlantic . . . . . . . . . 6

black.................... 6

Pacific.................... 6

hagfishes................ 6

hake, Carolina . . . . . . . . . . . . 22

gulf. .................... 22

longfin...................... 22

mud-see hake, white

Pacific..................... 22

silver................... 22

southern.................. 22

spotted .................. 22

squirrel.................. 22

white................... 22

hakes...................... 22

halfbeak .................... 20

California.................. 20

flying ....................... 20

hardhead ................. 20

longfin................... 20

ribbon..................... 20

31 halfbeaks.................. 20 
halfmoon

halfmoons.

halibut, arrowtooth-see flounder, arrowtooth

Atlantic 47

California

47

Greenland . .

northern-see halibut, Pacific

Pacific.

hamlet, butter

mutton.

see also grouper, marbled; grouper, Nassau

hammerhead, great.

scalloped.

smooth

handsawfish-see lancetfish, Pacific

hardhead.

see also croaker, Atlantic

hardtail-see runner, blue

harvestfish, northern southern.

headfish-see sunfish, ocean; mola, sharptail

herring - see also queenfish

Atlantic................. 10

Atlantic round............... 10

Atlantic thread ............... 10

bigeye-see ladyfish

blueback.

branch-see alewife

California round

dwarf.

flatiron .

glut-see herring, blueback

Japanese-see herring, round

lake.

see also cisco, least

moon-see mooneye

Pacific.

Pacific thread.

sea-see herring, Atlantic

skipjack .

summer-see herring, blueback

herrings.

highbrow-see prowfish

hind, brown-see graysby

red

rock. .

speckled

hitch

hogchoker

hogfish .

see also pigfish

red.

Spanish.

spotfin

hogmolly-see sucker, hog

10
32 hornpout--see bullhead

horse-mackerel-see mackerel, jack

houndfish

\section{I}

idiot-see rockfish, channel

inconnu.

\section{$\mathbf{J}$}

jack, almaco

bar.

black. .

bluntnose.................. 30

common-see jack, crevalle

cottonmouth............... 30

crevalle.................. 29

green..................... 29

horse-eye

29

Spanish-see runner, rainbow

yellow

jackfish-see pike, northern

jackknife-fish.................. 31

jack-ma-riddle-see ladyfish

jacks. . . . . . . . . . . . . . . . 29

jacksmelt................. 46

jambeau.................... 48

jawfish, banded...............44 41

dusky . . . . . . . . . . . . 42

longtail.......................... 41

mottled.............................. 41

Pensacola...................... 42

spotfin..................... 41

swordtail.................. 41

yellowhead................ 41

jawfishes.................. 41

jawless fishes................ 6

jenny, silver............... 30

jewfish ................... 25

see also sea bass, giant

black-see grouper, Warsaw

spotted-see jewfish

Joe Brown-see lizardfish

10 John Dory, American. . . . . . . . . . . . . 24

Johnny, tidepool-see sculpin, tidepool

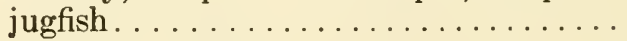

jumprock, bigeye. . . . . . . . . . . 17

black.................... 18

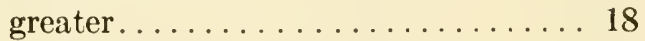

striped................. 18

\section{$\mathbf{K}$}

kelpfish-see also greenling, kelp

crevice.

giant. . . . . . . . . . . . . . . 42

island ..................... 42

scarlet. . . . . . . . . . . . . . . . . 42

spotted .................. . 42

striped . . . . . . . . . . . . . 42 
killifish, Ash Meadows.

banded.

bass - see killifish, striped

bayou.

bluefin.

California

common-see mummichog

diamond

goldspotted.

gulf

least. .

longnose

marsh

Pacific-see killifish, California

Pahrump.

plains.

pygmy..

Railroad Valley

rainwater.

Rio Grande.

Seminole.

speckled.

spotfin

striped

Waccamaw.

White River.

killifishes

king, silver-see louvar; tarpon

kingfish - see also croaker, white; mackerel, king

gulf .

northern

southern

king-of-the-salmon .

king-whiting-see kingfish

kiver-see pumpkinseed

kiyi .

kokanee.

see also salmon, sockeye

\section{$\mathbf{L}$}

ladyfish

Lafayette-see spot

lamprey, Allegheny brook

American brook.

Arctic

chestnut.

least brook.

mountain brook

northern brook.

Ohio.

Pacific.

river

sea.....

silver. .

southern brook.

threetoothed-see lamprey, Pacific

western brook.

6
21 lampreys.

$$
\text { northern sand. }
$$$$
\text { Pacific sand }
$$

lances, sand

Pacific..................... 13

lancetfishes.................. 13

lanternfishes................... 13

launce-see lance, sand

lawyer-sec burbot

leatherfish-see filefish

leatherjacket

lemonfish-see cobia

ling-see burbot; hake

lingeod.

lionfish . . . . . . . . . . . . . 36

liparid, continuous-finned-see snailfish, showy

Juan de Fuca-see snailfish, slipskin

prickly - see snailfish, prickly

ring-tailed-see snailfish, ringtail

shore-see snailfish, tidepool tadpole-see snailfish, tadpole

livebearers. . . . . . . . . . . . . . 22

lizardfish, California............. 13

inshore.................. 13

largescale................. 13

offshore .................. 13

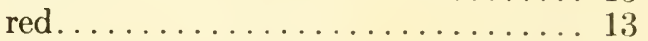

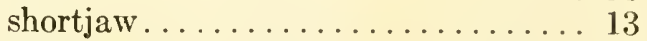

smallscale................. 13

lizardfishes ................. 13

loaches................. 18

loche-see burbot

logperch................... 29

logperch, Roanoke. . . . . . . . . . . 29

lookdown . . . . . . . . . . . . . . . 30

lord, brown Irish. . . . . . . . . . . . . . . 39

red Irish . . . . . . . . . . . . . . . 39

yellow Irish-see lord, brown Irish

louvar................... 35

6 louvars............................ 35

6 lumpfish.................. 41

6 lumpfishes................. 41

6 lumpsucker-see also lumpfish

Arctic . . . . . . ..............44 4

Atlantic spiny .............. 41

leatherfin.................. 41

Newfoundland spiny............ 41

Pacific spiny . . . . . . . . . . . . 41

smooth.................. 41

\section{M}

machete. . .............. 10

mackerel, American - see mackerel, Pacific 
mackerel, Atka. 38

Atlantic.

blue - see mackerel, Pacific

bull's eye-see mackerel, chub

chub

Florida-see cero

frigate.

green-see mackerel, Pacific

horse-see mackerel, jack; tuna, bluefin

jack.

king.

see also cero

Monterey Spanish.

Pacific.

painted-see cero

snake.

snap-see bluefish

Spanish

see also mackerel, jack

thimble-eye-see mackerel, chub

striped-see mackerel, Pacific

mackerels.

snake.

mackinaw-see trout, lake

mademoiselle - see perch, silver

madtom, black .

brindled

Carolina.

freckled.

least.

margined.

mountain

orangefin

slender.

speckled

tadpole.

major, sergeant

mako.

maneater-see shark, white

man-of-war fish.

manta, Atlantic

Pacific.

mantas.

margate

black .

maria-see burbot

marlin, barred-see marlin, striped

black .

blue.

striped

white.

marlin-spike

marlinsucker

maskinonge.

mayfish-see killifish, striped

medusafish

menhaden, Atlantic.

finescale. gulf-see menhaden, largescale largescale................... 10

midshipman, Atlantic............ 49

northern. . . . . . . . . . . . . . 49

$\operatorname{slim} \ldots . \ldots \ldots \ldots \ldots \ldots . \ldots . \ldots . \ldots 49$

miller's thumb - see sculpin, mottled

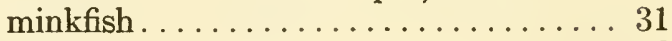

minnow, bluntnose . . . . . . . . 16

brassy................... 14

bullhead.................. 16

cutlips................... 13

cypress................... 14

desert-see pupfish, desert

Devils River.... . . . . . . . . . . . . . 13

fathead................... 16

Kanawha................. 16

Lake Eustis . . . . . . . . . . . . . . 21

loach.................. 17

Ozark................... 13

pugnose................. 16

redfin-see shiner, redfin

riffle.................... 16

roundnose................. 13

sheepshead .................. 21

silverjaw. . . . . . . . . . . . . . . 13

silvery.................. 14

$\operatorname{slim} \ldots \ldots \ldots \ldots \ldots \ldots \ldots \ldots$

spottail-see shiner, spottail

stargazing............... 16

suckermouth.............. 16

tallowmouth-see stoneroller

tonguetied . . . ............. 16

variegated-see minnow, sheepshead

minnows.................... 13

mobula, smoothtail............. 9

spinetail................. 9

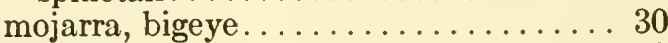

flagfin................... 30

mottled................. 30

slender.................... 30

spotfin................... 30

striped.................. 30

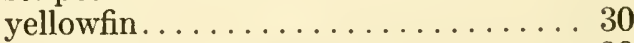

mojarras.................. 30

mola-see also sunfish, ocean

sharptail................... 49

slender. . . . . . . . . . . . . . . . . . 49

molas...................... 49

molly, Amazon................ 22

sailfin..................... 22

Molly Miller................. 43

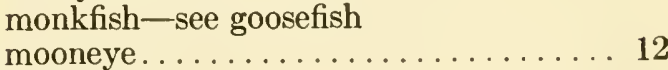

mooneyes. . . . . . . . . . . . . 12

moonfish-see also opah

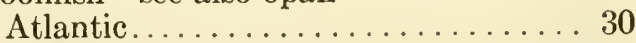

Pacific...................... 30

moray, blackedge ............ 19 
moray, California..

chain.

goldentail

green

purplemouth

pygmy.

reticulate.

speckled-see moray, spotted

spotted.

viper.

morays

mosquitofish

mossbunker - see menhaden, Atlantic

mousefish-see sargassumfish

muddler - see sculpin

mudfish - see bowfin; mummichog; killifish, striped; molly, sailfin

mudminnow, central

eastern

Olympic.

mudminnows

mudsucker, longjaw.

mullet, black.

blueback-see liza

common-see mullet, striped

fantail

grey-see mullet, striped

king - see goatfish, yellow

mountain .

redeye.

silver-see mullet, white

striped

white.

mullets

mummichog

muskellunge.

muttonfish-see pout, ocean

\section{$\mathbf{N}$}

needlefish, Atlantic.

California................... 20

flat...................... 20

Pacific-see needlefish, California

redfin...................... 20

needlefishes................. 20

nibblers.................... 32

niggerfish-see coney

night sergeant.

numbfish-see ray, lesser Atlantic

\section{O}

oarfish

oarfishes

oilfish

opah.

opahs

opaleye.
19

19

19

ouananiche.

oysterfish - see toadfish, oyster

\section{$\mathbf{P}$}

paddlefish................. 9

paddlefishes................... 9

palometa.................... 30

parrotfish, blue. . . . . . . . . . . . 34

bluelip..................... 34

bucktooth................. 34

red-see parrotfish, stoplight

emerald.................. 34

green-see parrotfish, stoplight

purple................... 34

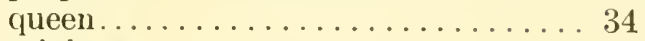

rainbow.................... 34

redband ................... 34

redfin..................... 34

redtail...................... 34

spotfin.................... 34

stoplight. . . . . . . . . . . . . 34

striped................... . 34

parrotfishes.................. 34

peamouth................ 14

pearleye, northern.............. 13

pearleyes................... 13

pearlfish . . . . . . . . . . . . 45

pearlfishes.................. 45

perch-see also seaperch; surfperch

bay-see perch, black

black.

see also blacksmith; opaleye

blue-see blacksmith; perch, black

blue-eye-see opaleye

button-see opaleye

Catalina-see blacksmith; opaleye

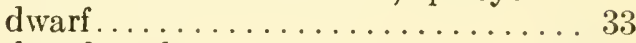

dwarf sand ................. 25

green-see opaleye; perch, yellow

kelp..................... 33

ocean.................... 37

Pacific Ocean................ 37

pile..................... 33

pirate. . . . . . . . . . . . . . . 24

reef...................... 33

Rio Grande. . . . . . . . . . . . . . 33

Sacramento . . . . . . . . . . . . 27

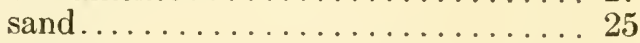

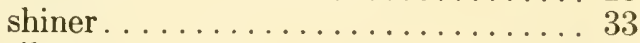

silver................... 31

splittail-see seaperch, white

stump--see sunfish, spotted

tule..................... 33

viviparous-see perch, tule

white................... 25

see also perch, silver; drum, freshwater yellow................... 29

zebra..................... 32 
perches

perches, pirate.............. 24

permit....................... 30

peto-see wahoo

pickerel-see also pike, northern; walleye

blue--see pike, blue

bulldog-see pickerel, redfin

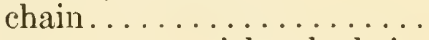

eastern-see pickerel, chain

grass . . . . . . . . . . . . .

mud-see pickerel, grass

redfin.................... 12

yellow-see walleye

pigfish .

pike, blue.

northern.

Oregon-see squawfish, northern

Sacramento--see squawfish, Sacramento

sand-see sauger

pikeblenny, bluethroat.

orangethroat.

pikeperch, blue-see pike, blue

pikeperch, yellow-see walleye

pikes

pilchard-see also sardine, Pacific

false.

redear-see sardine, redear

pilot, cockeye-see beaugregory; sergeant major

pilotfish

pinfish.

longspine - see porgy, longspine

spottail

pipefish, banded

barred

bay.

bull.

chain .

crested

dusky

fringed

gulf.

insular.

kelp.

northern

opossum

pugnose.

sargassum

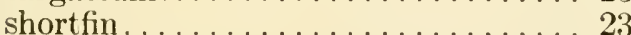

snubnose..................... 23

swivelhead ................ 23

whitenose . . . . . . . . . . . . . 23

pipefishes................... 23

plaice, Alaska. . . . . . . . . . . . . 47

American .

Canadian-see plaice, American

poacher, Atlantic beardless................... 40

Bering.................. 40

bigeye................. 40

blackfin...................... 40

blacktip................... 40

bluespotted...............41

cutfin.................... 41

fourhorn . . . . . . . . . . . . 40

longnose . . . . . . . . . . . . . . 40

northern spearnose............40

pricklebreast. . . . . . . . . . . . 40

pygmy................... 40

sawback.................... 40

southern spearnose ........... 40

stripefin . . . . . . . . . . . . . . 41

sturgeon . . . . . . . . . . . . . . . 40

tubenose................... 40

warty . . . . . . . . . . . . . . 40

poachers...................40

poggie - see perch, shiner

pogy - see surfperch, redtail; menhaden, Atlantic

pollack-see pollock

pollock

walleye................ 22

pollyfish--see parrotfish

pomfret...................... 30

bigscale.................... 30

pomfrets................... 30

pompano....................30

African.................... 29

California-see pompano, Pacific

common-see pompano

gafftopsail.

see also palometa

great-see permit

Irish

Pacific..................... 45

paloma................... 30

round-see permit

pompanos.

pompon-see margate, black

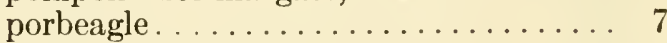

porcupinefish................ 49

porcupinefishes. . . . . . . . . . . . . 49

porgies.................. 32

porgy - see also scup; surfperch, redtail

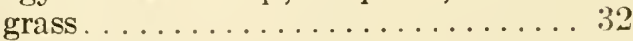

jolthead . . . . . . . . . . . . . . . 32

littlehead . . . . . . . . . . . . . . 32

littlemouth-see porgy, sheepshead

longspine. . . . . . . . . . . . . . 32

Pacific..................... 32

red...................... 32

saucereye................. 32

shad-see porgy, grass

sheepshead ................. 32

silver................. 32 
porgy, whitebone............... 32

porkfish.................... 30

pout, ocean................. 44

prickleback, black............... 44

bluebarred.................. 44

crisscross.................. 44

mosshead.................. 44

ribbon....................... 44

pricklebacks................. 44

priestfish — see rockfish, blue

prowfish.................... 45

prowfishes................... 45

puddingwife.................... 34

puffer, bandtail................. 49

bullseye.................... 49

checkered................... 49

marbled................... 49

northern . . . . . . . . . . . . . . . . . 49

oceanic.................... 49

sharpnose..................... 49

smooth.................... 49

southern..................... 49

puffers.................... 49

pug - see parrotfish, rainbow

pumpkinseed.................. 27

pupfish, Comanche Springs........... 21

desert.................. 21

Devils Hole. . . . . . . . . . . . . . . . 21

Leon Springs. . . . . . . . . . . . . 21

Nevada...................... 21

Owens River. . . . . . . . . . . . . . . 21

Red River................... 21

Salt Creek. . . . . . . . . . . . . . . . 21

sea-see minnow, sheepshead

\section{Q}

queenfish..................... 31

quillback.................... 17

quillfish .................... 44

quillfishes................... 44

$\mathbf{R}$

rabbitfish - see puffer, smooth; burrfish

rabirubia-see snapper, yellowtail

ragfish.

ragfishes.

rasher-see rockfish, vermilion

ratfish.

rat-tails.

raven, sea.

ray, bat-see stingray, bat

bullnose.

California butterfly

cownose.

devil

see also manta, Atlantic giant butterfly-see ray, spiny butterfly

lesser butterfly-see ray, smooth butterfly

lesser electric ................ 8

Pacific electric.............. 8

round sting - see stingray, yellow

smooth butterfly................. 9

southern eagle................ 9

spiny butterfly................ 9

spotted eagle................ 9

whip-see ray, bullnose

rays, eagle ................... 9

electric .................. 8

razorfish, green. . . . . . . . . . . 34

pearly................... 34

rosy ..................... 34

redeye- see bass, rock

redfish . . . . . . . . . . . . . . . . . . 37

see also drum, red; sheephead, California

little-see salmon, sockeye

redhorse, black. . . . . . . . . . . . . 18

blacktail.................. 18

Carolina.................. 18

copper................... 18

eastern-see redhorse, northern

golden.................. 18

gray..................... 18

greater................... 18

Lahontan . . . . . . . . . . . . . . . 17

Neuse...................... 18

northern.................. 18

river................ 18

shorthead................. 18

silver.................... 17

smallfin................. 18

suckermouth............... 18

V-lip . . . . . . . . . . . . . . . 18

reef-fish, gray .................. 33

yellowtail.................. 33

remora...................... 48

spearfish.................. 48

remoras..................... 48

ribbonfish-see cutlassfish, Atlantic; jackknife-fish

California-see king-of-the-salmon

polka-dot. . ................. 24

scalloped................... 24

9 ribbonfishes.................. 24

22 rig, sloop-see catfish, gafftopsail

39 rivercarp, bluntnose-see carpsucker, highfin

9 rivulus

roach - see also chub; bluegill

California.

robin, flying - see gurnard, flying

rock-eel-see blenny, rock

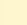


rockfish-see also bass, striped

Alaskan red-see rockfish, blackthroat

aurora.

banded-see rockfish, tiger

bigeye-see rockfish, sharpchin

black.

see also grouper, black; rockfish, blue

black-and-yellow

blackbanded-see rockfish, tiger

blackgill. . .

blackmouth.

blackthroat.

blue.

bronzespotted

brown

see also rockfish, calico

calico.

canary

China.

copper

coral-red.

cow.

dusky

fantail-see rockfish, canary

flag.

gopher.

grass . .

greenspotted

greenstriped.

halfbanded.

honeycomb

kelp

lobejaw-see rockfish, splitnose

longjaw-see perch, Pacific Ocean

longspine channel

northern

olive.

oliveback-see rockfish, stripetail

orange - see rockfish, canary

orange-red-see rockfish, rosethorn

orangespotted-see rockfish, quillback

pink

Puget Sound

pygmy

quillback

rasphead. .

redstripe

rosethorn

rosy

sharpchin

shortbelly

shortspine channel

silvergray .

slender-see rockfish, pygmy

speckled see also rockfish, quillback

spinycheeked - see rockfish, shortspine channel

splitnose................. 37

squarespot. . . . . . . . . . . 37

starry . . . . . . . . . . . . 37

stripetail................ 37

swordspine............... 37

tiger..................... 37

turkey red-see rockfish, rasphead

velvet-see gag

vermilion .

whitebelly

widow

yellowbacked-see rockfish, copper

yellowstripe - see rockfish, China

yellowtail .

rockfishes

rockhead .......................4 40

rockling, fourbeard . . . . . . . . . . 22

roller, sand . . . . . . . . . . . . . . . 24

ronco-see sailors choice

ronquil, Alaskan. . . . . . . . . . . . . . 42

northern .................... 42

rough.................... 42

smooth................... 42

ronquils................... 42

roosterfish.................. 30

rooter-see buffalo, black

rosefish-see also redfish

blackbelly.................. 36

rudd . . . . . . . . . . . . . . . . . . . . 17

rudderfish-see also chub, Bermuda

banded .................... 30

black-see barrelfish

brown-see medusafish

ruff, black. . . . . . . . . . . . . . . . . . 45

run, tailor-see bluefish

runner, blue. . . . . . . . . . . . . . . . . 29

hardtail-see runner, blue

rainbow

\section{$\mathbf{S}$}

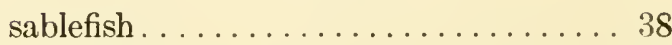

sablefishes... . . . . . . . . . . . . . . 38

sailfish, Atlantic. . . . . . . . . . . . . 35

Florida-see sailfish, Atlantic

Pacific. . . . . . . . . . . . . . . . . . . 35

sailorfish-see sculpin, sailfin

sailors choice. ................. 31 see also pinfish

salema................... 31

see also pinfish, spottail

salmon, Atlantic . .............. 11

black-sce cobia

blueback-see salmon, sockeye

chinook. .................... 11

chum..................... 11 
salmon, coho.

dog-see salmon, chum

fall-see salmon, chum

humpback-sce salmon, pink

jack-see walleye

king-see salmon, chinook

lake Atlantic

landlocked

see also salmon, Atlantic

medium red-see salmon, coho

pink.

quinnat-see salmon, chinook

red-see salmon, sockeye

Sebago

see also salmon, Atlantic

silver-see salmon, coho

sockeye.

spring - see salmon, chinook

tyee-see salmon, chinook

sanddab, longfin . .

mottled-see sanddab, Pacific

Pacific.

speckled.

sandfish-see also tilefish, sand

belted.

Pacific

sailfin

sandfishes.

sand lance - see lance

sardine, California - see sardine, Pacific

Pacific. .

redear

scaled.

Spanish

sargassumfish

sargo

saurel-see mackerel, jack

sauries....................... 20

saury, Atlantic. . . . . . . . . . . . . 20

Pacific................... 20

sawfish, largetooth . . . . . . . . . . . 8

smalltooth............... 8

sawfishes................. 8

scabbardfish................ 34

scad, bigeye................. 30

goggle-eye - see scad, bigeye

mackerel. . . . . . . . . . . . . . . . . . . 29

Mexican. . . . . . . . . . . . . . . 29

rough.................. 30

round . . . . . . . . . . . . . . . . 29

scads. . . . . . . . . . . . . . . . . 29

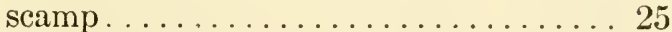

schoolmaster................ 26

scooter-see barracuda, Pacific

scorpionfish, California........... 37

finescale................. 37

goosehead
11

highfin

36

hunchback ................ 36

longfin................... . 36

longsnout. . . . ............ . 36

mushroom . . . . . . . . . . . . . 37

red..................... 36

reef . . . . . . . . . . . . . . . . 37

scalyhead ................. 36

smoothhead .................. 36

spinycheek.................. . 36

spotted .................. . . 37

scorpionfishes............... 36

scotehman-see lingeod

scrod-see cod, Atlantic; haddock

sculpin-see also scorpionfish, California

Aleutian-see sculpin, coastrange

Arctic . . . . . . . . . . . . . . . . . 40

Aretic staghorn... . . . . . . . . . . . 39

bald . . . . . . . . . . . . . . . . 39

banded . . . . . . . . . . . . . . 39 39

blackfin................... 40

bonehead................... 39

buffalo.................... 39

bull. . . . . . . . . . . . . . . 39

calico.................... . 39

California-see scorpionfish, California

cirrated-see sculpin, fluffy

coastrange. . . . . . . . . . . . . . 39

Columbia. . . . . . . . . . . . . . . . 39

coralline.................. 38

crested.................... 39

daddy-see sculpin, shorthorn

darter.................... . 40

see also sculpin, slim

deepwater-see sculpin, fourhorn

dusky ................... 39

eagle.................... 39

filamented-see sculpin, threadfin

fluffy . . . . . . . . . . . . . . . . 40 40

fourhorn . . . . . . . . . . . . . 40

fringed . . . . . . . . . . . . . . . 39

frogmouth . . . . . . . . . . . . . . . 39

giant marbled--see cabezon

globe-headed-see sculpin, mosshead

great. . . . . . . . . . . . . . . 40

grunt................... 40

hookear................... 38

Klamath Lake. . . . . . . . . . . . . . . . 39

lavender................. 40

lesser filamented-see sculpin, spotfin

longfin.................... . 39

longhorn . . . . . . . . . . . . . . 40

long-rayed-see sculpin, threadfin

mailed... . . . . . . . . . . . . . . 4 40

Malheur.................... 39

manacled .................. . 40

marbled ................... 39 
sculpin, marbled-see also cabezon

margined

mosshead

mossy-see sculpin, calico

mottled.................. 39

northern . . . . . . . . . . . . . . . . 39

Pacific staghorn.............. . 40

padded

see also sculpin, bonehead

pit-head.

39

Piute.

plumose-see sculpin, scalyhead

prickly

Puget Sound.

reticulate.

ribbed

riffle.

rosy.

rosylip.

rough.

roughback

roughcheek .

roughspine

round-headed-see sculpin, mosshead

roundnose-see sculpin, smoothhead

saddleback

sailfin .

scalyhead

sharpnose

shorthorn

Shoshone.

silverspotted.

slender

slim

slimy

smooth-see sculpin, Pacific staghorn

smoothgum

smoothhead

snubnose.

soft

spatulate

spinyhead

spinynose.

spoonhead

spotfin.

spurcheek

tadpole.

thornback

threadfin

tidepool

torrent.

twohorn

Wood River.

wooly

see also sculpin, spinyhead

yellowchin

sculpins .

scup seabass, black - see bass, giant sea white..................... 31

seahorse, dwarf . . . . . . . . . . . . 23

offshore................... 23

northern-see seahorse, spotted

Pacific.................... 23

spotted.................. 23

seahorses................. 23

sea mink-see minkfish

seaperch, barred - see surfperch, barred

black - see perch, black

blue-see seaperch, striped

brown-see perch, kelp

dusky - see perch, pile

forktail-see perch, pile

island. .

pink..................... 33

rainbow................... 33

rubberlip.................. 33

sharpnose................ 33

shiner-see perch, shiner

silver-see perch, pile

striped.

walleye-see surfperch, walleye

white.

yellowfin-see perch, pile

sea-poacher, deep-pitted-see rockhead window-tailed-sce poacher, northern spearnose

searcher................... . 42

searobin, armored............. 38

bandtail. . . ............... 38

barred..................... 38

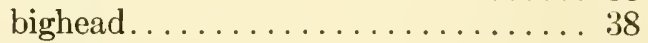

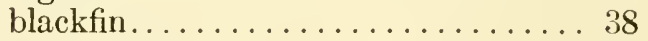

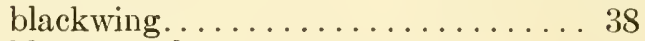

bluespotted................ 38

common-see searobin, northern

horned.................... 3s

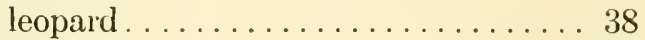

lumptail . . . . . . . . . . . . . . 38

Mexican................. 38

northern . . . . . . . . . . . . . 38

redwing - see searobin, striped

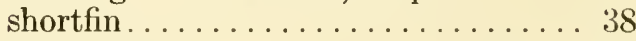

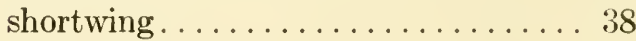

sicklefin.................... 38

slenderhead. ................. 38

spiny................... 38

streamer.................... 38

striped..................... . 38

searobins.................. 38

seasnail . . . . . . . . . . . . . 41

gelatinous................... 41

Greenland.................. 41

striped ................... 41

seatrout, bastard-see seatrout, silver gray - see weakfish 
seatrout, greenling - see greenling, kelp sand.

silver.

spotted

white-see seatrout, sand

sennet, northern .

southern.

señorita.

sergeant major.

sergeant, night

rock-see sergeant, night

sergeantfish - see cobia

seven-eleven-see perch, shincr

shad, Alabama.

American .

gizzard

golden - see herring, skipjack

hickory see also shad, gizzard

\section{Ohio}

threadfin.

white-see shad, American

yellowfin. .

shanny

Arctic.

radiated.

shark, Atlantic angel

Atlantic sharpnose

basking.

bay

bignose

blackfin-see shark, blacktip

blacknose

blacktip . .

blue.

see also shark, bonito

bonito.

bonnetnose - see bonnethead

bramble. .

brown-see shark, sandbar

brown cat.

bull. .

bullhead-see shark, horn

cat-see shark, leopard; shark, nurse

cow-see shark, sixgill

cub-see shark, bull

dusky .

false cat

filetail cat.

finetooth.

fox-see shark, thresher

frill

great blue-see shark, blue

great white-see shark, white

Greenland

hammerhead-see hammerhead

horn

kitefin. lemon.................. 7

leopard

sce also shark, tiger

mackerel-see mako; shark, salmon

maneater-see shark, white

mud-see shark, sixgill

narrowtooth

night.

nurse

see also shark, Greenland

Pacific angel............... 8

Pacific sharpnose.............. 7

Pacific sleeper.

pilot-see rudderfish, banded

rabbit-see ratfish

ridgeback-see shark, sickle

roundnose.................. 7

salmon................... 7

sand ..................... 6

sandbar................... 7

sevengill. ................ 6

shovelnose-see bonnethead; guitarfish, shovelnose

sickle................. 7

silky................. 7

sixgill................. 6

sleeper-see shark, Greenland

smalltail................ 7

smooth-tooth-see shark, finetooth

soupfin

spinner

spotfin-see shark, blacktip

swell

swiveltail-see shark, thresher

thresher................... 6

tiger..................

whale................... 7

white................. 7

whitetip.................. 7

sharks, angel............... 8

cat...................... 7

cow ...................... 6

$\operatorname{dogfish} \ldots \ldots \ldots \ldots \ldots \ldots . \ldots . \ldots$

frill $\ldots \ldots \ldots \ldots \ldots \ldots \ldots \ldots \ldots$

hammerhead ............... 7

horn..................... 6

mackerel................ 6

nurse ...................... 7

requiem.................... 7

sand.................... 6

whale................... 7

sharksucker..................448

whitefin.................. 48

8 sheefish-see inconnu

sheephead, California.............. 34

6 sheepshead.................. 32

8 shellcracker-see sunfish, redear

7

7
7
7
8
7
8

.

.

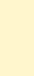

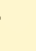

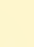

7

7

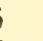

\begin{tabular}{l}
7 \\
8 \\
\hline
\end{tabular}

8

8

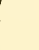

6

7


shellfish-see trunkfish

shiner, Alabama............... 15

Altamaha................... 16

Arkansas River . . . . . . . . . . . 15

bandfin................. 16

bigeye................. 15

bigmouth............... 15

blackchin. .............. 15

blacknose................. 15

blackspot............... 15

blacktail. . . . . . . . . . . . . 16

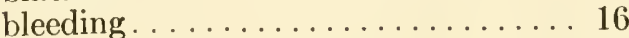

blue.................... 15

bluenose. . . . . . . . . . . . . . . . 16

bluestripe................ 15

bluntface................ 15

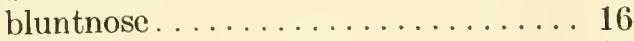

Brazos................... 15

bridle................. 15

broadstripe............... 15

burrhead.................. 14

cherryfin................ 16

Chihuahua............... 15

chub.................. 16

coastal................. 16

colorless . . . . . . . . . . . . . 15

comely................... 14

common................ 15

Coosa . . . . . . . . . . . . . . 16

crescent................ 15

dusky .................. 15

emerald................... 14

fieryblack. . . . . . . . . . . . 16

flagfin . . . . . . . . . . . . . 16

ghost................... 15

golden .................. 14

greenfin................... 15

greenhead ................ 15

highfin................. 14

highscale.................. 15

ironcolor.................. 15

Kiamichi................. 15

lake-see shiner, emerald

longnose . . . . . . . . . . . . . . . . 15

$\operatorname{mimic} \ldots \ldots \ldots \ldots \ldots \ldots \ldots$

mirror ................. 16

mountain................ 15

New River. . . . . . . . . . . . . . . . 16

Ocmulgee................ 15

Ohoopee................... 15

Ozark..................... 15

pallid.................... 14

pinewoods............... 15

plains...................... 15

plateau..................... 15

popeye................... 14

pretty ................... 15

Proserpine............... 16 pugnose................. 14

rainbow................. 15

red.................... 15

redfin................... 16

redlip.................. 15

Red River................. 15

redside.................. 17

ribbon.................. 15

Rio Grande. . . . . . . . . . . . 15

river................. 15

rosefin.................... 14

rosyface............... 16

rough................. 15

Sabine.................... 16

saffron................... 16

sailfin................... 15

sand .................. 16

sandbar.................. 16

satinfin.................. 14

sharpnose.................. 15

silver................... 16

silverband............... 15

silverstripe. ............... 16

smalleye ................. 15

spotfin.................. 16

spottail.................. 15

stargazing. . . . . . . . . . . 16

steelcolor.................. 16

swallowtail. ............... 16

taillight................. 15

Tamaulipas................. 15

Tennessee................... 15

Texas..................... 14

Topeka.................. 16

tricolor . . . . . . . . . . . . . 16

warpaint................. 15

wedgespot................. 15

weed . . . . . . . . . . . . . . 16

white...................... 14

whitefin................. 15

whitemouth ............. 14

whitetail................. 15

yellow-see perch, shiner

yellowfin................. 15

silverfish-see tarpon

silver-rag. ................ 45

silverside, Atlantic . . . . . . . . . . . 46

brook..................... 46

hardhead..................... 46

key.................... 46

Mississippi.................. . 46

reef.................... 46

rough................... 46

tidewater................ 46

Waccamaw................ 46

silversides................. 46

silverspot-see sculpin, silverspotted

singingfish-see midshipman 
skate, Alaska.

Aleutian.

barndoor . .

Bering.

big.

black

briar-see skate, clearnose

California

clearnose.

common-see skate, little

flathead.

freckled.

little.

longnose

ocellate.

Pacific prickly-see skate, starry

prickly - see skate, starry; skate, thorny

rosette...

roughtail.

roundel

sandpaper-see skate, black

smooth.

spinytail.

spotted-see skate, winter

spreadfin.

starry.

thorny

winter

skates

skilfish

skilletfish

skipjack-see also herring, skipjack; tuna, skipjack; saury, Atlantic; silverside, brook; bluefish

black.

wavyback

skipper-see saury, Pacific

sleeper, bigmouth.

blue.

emerald.

fat.

masked

spinycheek

spotted.

sleepers.

smelt, American

Arctic.

freshwater-see smelt, pond

longfin.

night.

pond.

Sacramento.

silver-see smelt, surf

surf

whitebait

smelts

deepsea
8 smoothhound, brown

8

8

8

8

8

8

8

8

8

8

8

8

Florida................. 7

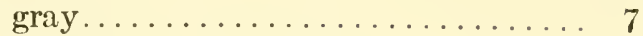

sicklefin................ 7

smoothtongue, California........... 12

snailfish, Bering................ 41

lobefin................... 41

longfin.................. 41

marbled...................44 41

polka-dot................ 41

prickly...................44 41

ribbon.................44 41

ringtail . . . .............. 41

showy ................... 41

slimy................... 41

slipskin................. 41

spotted..................44 41

tadpole................... 41

tidepool................... 41

9 snailfishes................. . 41

9 snakeblenny, fourline............ 44

Pacific.................... 44

8 snakefish.................. 13

9 snapper-see also bluefish

black................... 26

8 blackfin................ 26

9 blue-see bluefish

8 Caribbean red............... 26

8 Cuban-see snapper, cubera

cubera.................... 26

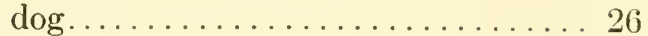

glasseye................. 26

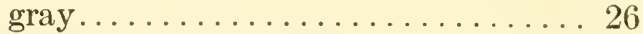

gunmouth-see snapper, blackfin

hog-see hogfish

lane.................... 26

mahogany............... 26

mangrove--see snapper, gray

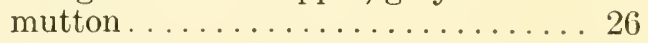

queen..................... 26

red.................... 26

see also rockfish, rasphead

silk..................... 26

spot-see snapper, lane

vermilion . . . . . . . . . . . . 26

yellowtail. ............... 26

snappers................. 26

snipefish, longspine............ 23

slender.................... 23

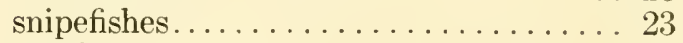

snook..................... 25

Cuban-see snook, tarpon

little..................... 24

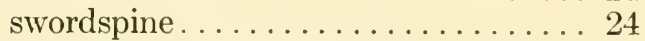

tarpon.................... 25

snooks...................... 24

soapfish................... 25

freckled.................. 26 
soapfish, spotted . . . . . . . . . 26

soldierfish, bigeye ............. 24

blackbar.................. 24

cardinal.................... 24

soldierfishes............. . . . . . 24

sole, American-see hogchoker

arrowtooth-see flounder, arrowtooth

Bellingham-see sole, butter

bigmouth

broadfin-see sole, rock

butter.................... 47

C-O . . . . . . . . . . . . . 47

curlfin................... 47

deepsea.................... 47

Dover.................... 47

English................ . 47

see also sole, petrale

fantail. . . . . . . . . . . . . . . . 47

flathead...................... 47

fringe - see sole, sand

gray-see flounder, winter; flounder, witch

lemon-see flounder, winter; sole, English

lined.

naked.

petrale

pointednose-see sole, English

rex.

rock.....................44 47

rough-see plaice, American; sole, slender

roundnose-see sole, petrale

sand.

scalyfin-see sole, butter

scrawled

slender.

slippery-see sole, Dover

yellowfin.

soles.

sourbelly - see saury, Pacific

sourfish - see saury, Pacific

spadefish, Atlantic.

Pacific.

spadefishes.

spearfish, longbill .

shortbill

spikedace.

spikefishes.

spinedace, Little Colorado $\cdots \cdots \cdots \cdots$

splittail

spoonbill-see paddlefish

spot. .

squaretail, bigeye

smalleye

squaretails

squawfish, Colorado

Columbia-see squawfish, northern common-see squawfish, northern

northern . . . . . . . . . . . . .

Oregon-see squawfish, northern

Sacramento............... 16

Umpqua.................... 16

squeteague, gray-see weakfish

sand-see seatrout, sand

silver - see seatrout, silver

spotted-see seatrout, spotted

squirrelfish ................... 24

deepwater.................. 24

dusky.................... 24

longjaw.................. 24

longspine................ 24

reef ..................... 24

squirrelfishes . . . . . . . . . . . . 24

stargazer, arrow ............... 42

freckled................... 42

lancer..................... 42

northern ................... 42

saddle. . . . . . . . . . . . . . . . . 42

sand ................... 42

smooth . . . . . . . . . . . . . 42

southern................ 42

stargazers.................. 42

sand .................... 42

starsnout, gray . . . . . . . . . . . 40

spinycheek................. 40

stickleback, brook.............. 23

common-see stickleback, threespine

fourspine................ 23

ninespine................ 23

threespine................ 23

twospine................. . 23

sticklebacks................ 23

stingaree - see stingray

stingray, Atlantic.............. 9

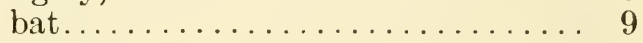

bluntnose................. 9

diamond .................. 9

pelagic................. 9

roughtail................... 9

round.................. 9

southern............... 9

yellow................. 9

stingrays.................. 9

stonecat.................. 18

stoneroller. . . . . . . . . . . . . 13

see also sucker, hog

Mexican.................. 13

streaker-see bass, yellow

studfish, northern ............... 21

southern ................. 21

stumpknocker-see sunfish, spotted

sturgeon, Atlantic.............. 9

common-see sturgeon, Atlantic

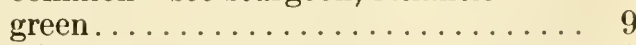

lake $\ldots \ldots \ldots \ldots \ldots \ldots \ldots \ldots . \ldots . \ldots$ 
sturgeon, Pacific-see sturgeon, white pallid.

river-see sturgeon, shovelnose

shortnose.

shovelnose

white.

sturgeons

sucker, Alabama hog

black-see sucker, northern hog

blackfin.

blue. .

bluehead

bridgelip

coarsescale-see sucker, largescale

Columbia finescale-see sucker, bridgelip

Columbia largescale-see sucker, largescale

Columbia smallscale-see sucker, bridgelip

common-see sucker, white

finescale-see sucker, longnose

flamelmouth . . . . .......... 17

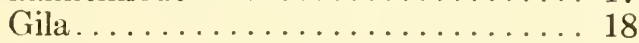

green.................... 18

harelip................. 17

highfin-see carpsucker, highfin

humpback................. 18

June. . . . . . . . . . . . . . . . . . . . . 17

Klamath largescale............. 17

Klamath smallscale............. 17

Lahontan . . . . . . . . . . . . . . . 18

largescale................... 17

longnose... . . . . . . . . . . . . . 17

Lost River. . . . . . . . . . . . . . . . 17

Missouri-see sucker, blue

Modoc....................... 17

mountain................ 18

northern-see sucker, longnose

northern hog .

razorback-see sucker, humpback

Rio Grande

river-see redhorse, northern

Roanoke hog............... 17

rustyside.................. 18

Sacramento................. 17

Santa Ana. . . . . . . . . . . . . 18

shortnose................ 17

Sonora................... 17

spearfish-see marlinsucker

spotted.................. 17

sturgeon-see sucker, longnose

sweet-see chubsucker

Tahoe

torrent

Utah

Warner.

webug. . western-see sucker, Sacramento

white. .

White River.

suckerfish-see also remora

slender.

white.

suckers.

sunfish, banded

banded pygmy

bantam ................... 27

blackbanded................. 27

bluespotted.................. . 27

common-see pumpkinseed

dollar.................... 27

Everglades pygmy . . . . . . . . . . . 27

green ..................... 27

longear . . . . . . . . . . . . . . . 27

mud.................... 27

ocean................... . 49

Okefenokee pygmy ............. . 27

orangespotted .............. 27

peacock-see flier

red-see sunfish, spotted

redbreast.................. 27

redear..................... 27

round-see flier

sharptail ocean-see mola, sharptail

spotted.

yellowbelly - see sunfish, redbreast

sunfishes................. 27

surffish-see corbina, California; croaker, yellowfin

surfperch, barred.............. 33

calico... . . . . . . . . . . . . . . . 33

redtail................... 33

silver. . . . . . . . . . . . . . . . . 33

spotfin.................... 33

walleye................... 33

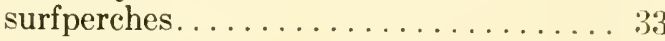

surgeon, ocean................. . 34

surgeonfish, gulf . . . . . . . . . . . 34

surgeonfishes................. 3t

swampfish................. 22

sweeper, glassy . . . . . . . . . . . . . . 32

sweepers................... . . 32

swellfish-see puffer

swiveltail-see shark, thresher

swordfish

broadbill-see swordfish

swordfishes.

\section{$\mathbf{T}$}

tai-see porgy, Pacific

tambor-see rockfish, rasphead

tang, blue

ocean-see surgeon, ocean

tarpon..................... 10

tarpons................... 10 
tattler.

tautog

taylor - see bluefish

tench.

tenpounder-see ladyfish; machete

tetra, Mexican................. 13

tetras........................ 13

thornback................ 8

thornhead-see rockfish, channel

threadfin, Atlantic.

Pacific.

yellow

46

threadfins

threadfish-see pompano, African

thresher, bigeye................
tiger, sand-see shark, sand

tilefish

blackline.

sand.

tilefishes

timucu.

toadfish, gulf .

leopard.

oyster.

toadfishes

tobacco box-see skate, little

togue - see trout, lake

tolly, ocean-see durgon, black

tomcod-see also hake, southern

Atlantic. .

Pacific.

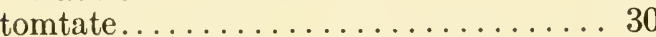

tonguefish, blackcheek. . . . . . . . . 48

California..................... 48

deepwater................ 48

largescale.................. 48

longtail. ................. 48

northern................... 48

offshore................ 48

pygmy................. 48

spottail................... 48

spottedfin................... 48

tonguefishes................ 48

topminnow-see also mosquitofish

banded.

blackspotted .................. 21

blackstripe................ 21

Gila... . . . . . . . . . . . . . . . . 22

golden................... 21

plains.................. 21

saltmarsh ................. 21

starhead .................. 21

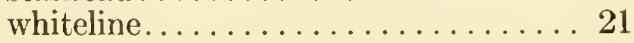

topsmelt................. 46

torpedo, Atlantic.............. 8

Pacific-see ray, Pacific electric

treefish. triggerfish, common-see triggerfish, gray finescale.................. 48

gray................... 48

ocean.................. 48

queen..................... 48

redtail.................. 49

rough................... 48

sargassum................. 49

spotted.................. 48

triggerfishes................. 48

tripletail.................. 26

tripletails. . . . . . . . . . . . 26

trout, bastard-see seatrout, silver

blackspotted - see trout, cutthroat

blueback................... 12

brook.................... 12

brown . . . . . . . . . . . . . . 12

bull-see Dolly Varden

cutthroat................... 11

Eastern brook - see trout, brook

Gila................... 11

golden................... 11

grey-see trout, lake; weakfish

Kamloops-see trout, rainbow

lake.

Mackinaw-see trout, lake

mountain-see trout, cutthroat

Quebec red

rainbow.

rock-see greenling, kelp

sand-see seatrout, sand

sea-see also greenling, kelp; seatrout, spotted

speckled-see trout, brook

steelhead..............

see also trout, rainbow

summer-see weakfish

Sunapee.

see also char, Arctic

white--see char, Arctic; seatrout, sand winter-see seatrout, spotted

trout-perch.

Columbia-see sand roller

trout-perches.

trouts..................... 11

trumpetfish................. 23

trumpetfishes................ 23

trunkfish..................... 49

horned-see cowfish

smooth . . . ............. 49

spiny .................... 49

spotted .................. 49

trunkfishes . . . . . . . . . . . . . . . . 49

tube-eye. . . . . . . . . . . . . . . . . 24

tube-eyes. . . . . . . . . . . . . . . 24

tube-snout. . . . . . . . . . . . . . 23

tube-snouts.................. 23 
tuna, Atlantic-see tuna, bluefin

bigeye................... 35

blackfin................... 35

bluefin.................... . 35

little.................... . 34

skipjack . . . . . . . . . . . . 34

striped-see tuna, skipjack

yellowfin.

tunas........................ 34

turbot, California-see sole, curlfin

$\mathrm{C}-\mathrm{O}$ - see sole, $\mathrm{C}-\mathrm{O}$

common-see triggerfish, gray

curlfin-see sole, curlfin

diamond.

hornyhead.

Nassau - see triggerfish, queen sharpridge - see turbot, hornyhead spotted.

tyee-see salmon, chinook

\section{$\mathbf{V}$}

Varden, Dolly

\section{W}

wahoo

snake-see wahoo

walleye.

warmouth.................. 27

Warsaw-see grouper, Warsaw

weakfish..................... 31 spotted-see seatrout, spotted

weatherfish, oriental.

wenchman.................. 26

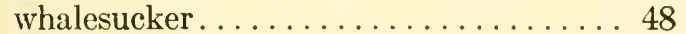

whiff, bay. ................... 46

hormed.................. 46

spotted................. 46

whipray - see ray, spotted eagle

whitebait-see silverside

whitefish-see also wolffish, Atlantic

Bear Lake.

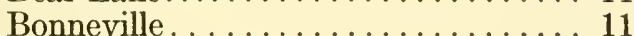

broad...................... 11

humpback................ 11 lake..................... 11

Menominee-see whitefish, round mountain . . . . . . . . . . . . . . . 11

ocean..................... 29

pygmy.................. 11

Rocky Mountain-see whitefish, mountain

round..................... 11

whitefishes. . . . . . . . . . . . 11

whiting-see also hake, silver; pollock, walleye

California-see corbina, California sand-see windowpane

windowpane................ 47

wolf-eel. . . . . . . . . . . . . . 43

wolffish-see also lancetfish, Pacific

Atlantic................... 43

Bering . . . . . . . . . . . . . . . . . 43

northern .................. 43

spotted .................... 43

wolffishes . . . . . . . . . . . . . . . . . 43

wormfish, pink.............. . 36

pugjaw...................... 36

wormfishes . . . . . . . . . . . . . . 36

woundfin.................. 16

wrasse, blackear. . . . . . . . . . . . 34

clown...................... 34

creole.................... 33

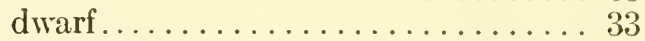

greenband................. 33

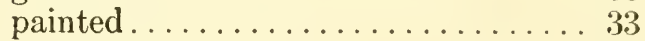

rock....................... 34

yellowhead................... 33

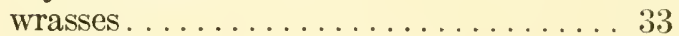

wreckfish.................. 25

wrymouth................. 44

dwarf..................... 44

giant.................... 44

$\mathbf{Y}$

Y-blenny.................... 44

yellowtail.................. 30

see also flounder, yellowtail; snapper, yellowtail; perch, silver

yowler-see pout, ocean

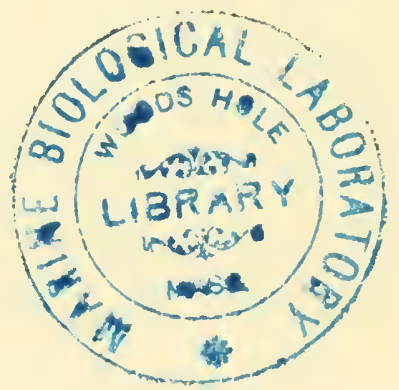




\section{PART III}

\section{Index to Scientific Names}

Names of classes, orders, and families appear in roman; those of genera and species in italies.

\section{A}

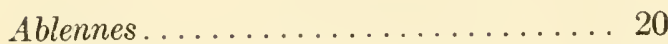

Abudefduf.................................. 33

abyssicola, Prosopium............. 11

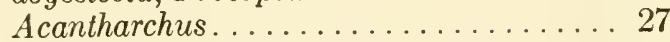

Acanthemblemaria.............. 42

acanthias, Squalus............... 8

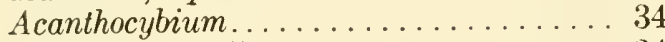

Acanthopterygii................ 24

Acanthostracion-see Lactophrys

Acanthuridae................. 34

Acanthurus.................... 34

Achirus................. 47

Acipenser.................. 9

Acipenseridae............... 9

Acipenseriformes............... 9

acipenserinus, Agonus............ . 40

ackleyi, Raja.............. 8

Acrocheilus................. 13

acronotus, Carcharhinus........... 7

acros, Eremichthys. ............... 13

aculeatus, Gasterosteus.............. 23

Halieutichthys................ 50

Prognathodes.................. 32

Stenotomus-see chrysops, S.

acuminatus, Equetus . . . . . . . . . . 31

Etrumeus. . . . . . . . . . . . . . 10

Myrichthys . . . . . . . . . . . . . . 19

acus, Strongylura................. 20

acuticeps, Clinocottus.............. 39

Etheostoma . . . . . . . . . . . . . 27

acutus, Fodiator. . . . . . . . . . . . . 21

Acyrtops................... 48

Adinia..................... 21

adscensionis, Epinephelus........... 25

adspersus, Tautogolabrus............. 34

aeglefinus, Melanogrammus......... 22

aeneus, Myoxocephalus........... 40

aenigmaticus, Icosteus. . . . . . . . . 45

aepyptera, Lampetra. . . . . . . . . . 6

aestivalis, Alosa................ 10

Hybopsis................ 14

Aetobatus.................. 9

afer, Alphestes............... 25

affinis, Atherinops............. 46
Chilomycterus................ 49

Elops....................... 10

Gambusia................... 22

Hirundichthys................ . 21

agassizi, Chologaster.............. 22

Scorpaena................... 36

aggregata, Cymatogaster. . . . . . . . 33

Agnatha..................... 6

Agonidae ..................... 40

Agonopsis...................440

Agonostomus................ 46

Agonus...................... 40

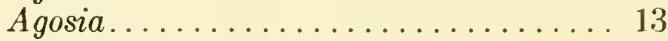

aguabonita, Salmo............... 11

Ahlia......................... 19

alabamae, Alosa................. 10

alalunga, Thunnus................ 35

alascana, Asterotheca.............. 40

alascanus, Sebastolobus.............. 38

alatus, Prionotus.................. 38

albacares, Thunnus.............. 35

albeolus, Notropis. . . ............. 14

albescens, Remorina..............44

albicans, Istiophorus............. 35

albida, Makaira............... 35

albigutta, Kathetostoma.............. 42

Paralichthys.................. 47

albirosiris, Corythoichthys........... 23

albolineatus, Fundulus. . . . . . . . . . 21

alborus, Notropis. . . . . . . . . . . . . . 14

Albula......................... 10

Albulidae. . . . . . . . . . . . . . . . . . . 10

album, Haemulon. . . . . . . . . . . . . 30

albus, Scaphirhynchus. . . . . . . . . . 9

Alectis...................... 29

alepidota, Chaenopsis. . . . . . . . . . 42

alepidotus, Peprilus.............. 45

Alepisauridae. . . . . . . . . . . . . . 13

Alepisaurus.................. 13

aleutensis, Lyconectes. . . . . . . . . . . . . . 44

aleutianus, Sebastodes............. 37

aleutica, Raja.................... 8

alcuticus, Cottus.................. 39

Allanetta.................... 46

alleni, Rathbunella............. 42

alletteratus, Euthynnus............ 34 
Alloclinus .

Allolumpenus.

Allosmerus

Allotriognathi

Alopias.

Alosa.

alosoides, Hiodon

alpenae, Coregonus

Alphestes.

alpinus, Salvelinus.

alta, Pristigenys.

altavela, Gymnura

altima, Carcharhinus

altipinnis, Notropis.

altivelis, Sebastolobus

alutaceus, Acrocheilus.

Alutera

alutus, Apogon.

Sebastodes

amabilis, Notropis

Ambloplites.

amblops, Hybopsis

Amblyopsidae.

Amblyopsis.

amblyrhynchus, Hemicaranx

Ameiurus-see Ictalurus

americana, Dasyatis

americanus, Ammodytes

Esox americanus

Hemitripterus.

Istiophorus-see albicans, $I$.

Lophius. .

Macrozoarces.

Menticirrhus.

Polyprion

Pseudopleuronectes.

Roccus.

Amia

Amiidae

Amiiformes.

Ammocrypta

Ammodytes.

Ammodytidae

amnis, Notropis

amoenus, Notropis.

Amphiodon-see Hiodon

Amphistichus.

Anacanthini .

anale, Hyperprosopon

analis, Clinocottus.

Lutjanus .

analogus, Epinephelus. . . . . . . . . . . . 25

analostanus, Notropis. . . . . . . . . . . 14

Anarchias.

Anarhichadidae.

Anarhichas.

Anarrhichthys . anatirostris, Bembrops

Anchoa...................... 11

anchovia, Sardinella............. 10

Anchoviclla.................... 11

Ancylopsetta................... 46

andersoni, Pristipomoides........... 26

Anguilla ...................... 19

anguillicaudatus, Misgurnus.......... 18

Anguillidae.................... 19

Anguilliformes................... 19

anguineum, Chlamydosclachus....... 6

angustirostris, Tetrapturus.......... 35

Anisotremus.................. 30

anisurum, Moxostoma............. 17

annae, Cottus. . . . . . . . . . . . . . . 39

annularis, Pomoxis. . . . . . . . . . . 27

Prionodes................... 25

annulatus, Sphaeroides............. 49

anogenus, Notropis. . . . . . . . . . . . . 14

anomalum, Campostoma............ 13

Anoplagonus..................440

Anoplarchus...................444

Anoplopoma................. 38

Anoplopomatidae. . . . . . . . . . . . 38

Antennariidae. . . . . . . . . . . . . 50

Antennarius................... 50

9 antennatus, Chilomycterus. . . . . . . . . 49

41 Anthias....................... 25

12 Antigonia.................... 24

39 antillarum, Chilomycterus............ 49

Monolene...................44 47

Apeltes..................... 23

aphododera, Dysomma.............. 20

Aphredoderidae.................. 24

Aphredoderus.................. 24

Aplodinotus..................... 31

Apodes......................... 19

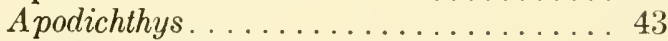

apodus, Lutjanus. . . . . . . . . . . . . . 26

Apogon ....................... 26

Apogonidae................... 26

approximans, Polydactylus........... 46

Aprionodon.................. 7

Apristurus................ 7

Aprodon..................... 44

Apsilus..................... 26

Aptocyclus................... 41

aquosus, Scophthalmus........... 47

Archoplites..................... 27

Archosargus ................... 32

arcticus, Thymallus................. 12

Trachipterus.................. 24

arctifrons, Calamus................. 32

Citharichthys................. 46

Arctogadus..................... 22

Arctoscopus...................... 41

arctus, Syngnathus................ 23 
arcuatus, Pomacanthus .

ardens, Catostomus

Notropis

arenarius, Cynoscion.

arenatus, Priacanthus .

Rypticus

argentea, Sphyraena

argenteum, Hyperprosopon.

argenteus, Amphistichus .

Diplodus.

Eucinostomus.

Argentina .

Argentinidae.

argentissimus, Plagopterus.

argi, Centropyge .

Argyreiosus-see Selene

Ariidae.

ariommum, Moxostoma.

ariommus, Notropis.

Arioscopus

Ariosoma.

armatus, Leptocottus

Artediellus. .

artedii, Coregonus .

Artedius.

Ascelichthys.

ascensionis, Holocentrus.

Asemichthys .

asper, Cottus

aspera, Acanthemblemaria

Limanda

asperifrons, Notropis.

asperilinguis, Anthias.

asperrimus, Cottus.

Aspicottus-see Enophrys

Aspidophoroides.

asprella, Ammocrypta .

asprellus, Radulinus. .

asprigene, Etheostoma. .

Asterotheca.

Astronotus.

Astroscopus.

Astyanax.

Atheresthes.

Atherinidae

atherinoides, Chriodorus .

Notropis

Atherinomorus.

Atherinops.

Atherinopsis

atinga, Chilomycterus

atlantica, Emblemaria .

Megalops.

Scorpaena.

atlanticus, Liparis.

Ophioblennius.

Tetragonurus .
Thunnus...

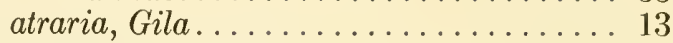

atratulus, Rhinichthys............. 16

atricauda, Symphurus............. 48

atripes, Phanerodon............. 33

atripinne, Etheostoma . . . . . . . . . 27

Moxostoma................... 17

atrocaudalis, Notropis............. 15

atromaculatus, Semotilus............ 17

atropurpureus, Xiphister............ 44

atrovirens, Sebastodes............. 37

audax, Makaira.................. 35

audens, Menidia................ 46

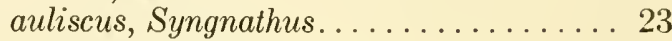

Aulorhynchidae................ 23

Aulorhynchus.................. 23

Aulostomidae.................... 23

Aulostomus.................... 23

aurantiaca, Percina.............. 29

auratus, Carassius................ 13

Mullus................... 31

aureolum, Moxostoma-see macrolepidotum, $M$.

aureorubens, Pronotogrammus........ 25

aureus, Pomacanthus................ 32

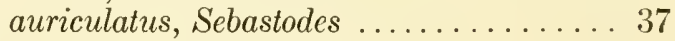

aurifrons, Opisthognathus. . . . . . . . . 41

auritus, Lepomis. ................ 27

aurofrenatum, Sparisoma........... 34

aurolineatum, Bathystoma.......... 30

aurolineatus, Apogon............. 26

aurora, Micrometrus............... 33

Sebastodes.................. 37

aurorubens, Rhomboplites........... 26

australis, Remora................ 48

autumnalis, Coregonus.............. 11

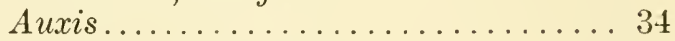

avalonis, Antennarius............. 50

averruncus, Kathetostoma........... 42

Awaous..................... 35

axillare, Sparisoma.............. 34

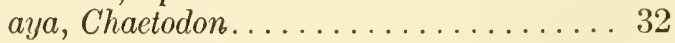

ayresi, Lampetra............... 6

Ayresia-see Chromis

azurea, Hermosilla............... 32

azureus, Carcharhinus............ 7

\section{B}

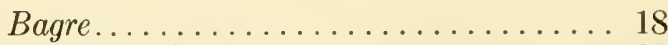

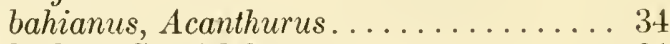

baileyi, Crenichthys..............21

Notropis................ 15

bairdi, Callionymus............. 42

Cottus...................... 39

Nezumia................... 22

Notropis.................. 15

Bairdiella.................... 31 
bajonado, Calamus.

balao, Hemiramphus.

20

baldwini, Prionodes.

25

Balistes.

Balistidae

48

48

balteatus, Richardsonius............. 17

barbata, Brotula. . . . . . . . . . . . . 45

Pallasina.

barbatum, Lyconema

Barbulifer

\section{0}

44

barracuda, Sphyraena

barratti, Etheostoma...

bartholomaei, Caranx.

bartoni, Aspidophoroides

Bascanichthys.

Bathyagonus

bathybius, Embassichthys.

Bathygobius .

Bathylagidae.

Bathymaster.

Bathymasteridae.

bathyphilus, Halichocres.

Bathystoma.

Batoidei.

Batrachoididae.

Batrachoidiformes.

bdellium, Ichthyomyzon.

beani, Ammocrypta

Prionotus.

beldingi, Cottus.

Bellator.

bellica, Hybopsis.

bellus, Notropis.

belone, Tetrapturus.

Belonidae.

Beloniformes ..................... 20

Bembrops...................... 42

bendirei, Cottus................... 39

bergi, Scorpaena.................. 36

beringiana, Polypera............. 41

bermudensis, Carapus............. 45

Holacanthus.............. . . . . . 32

Hypleurochilus............... 43

Beryciformes..................... 24

Berycoidei.................... 24

beryllina, Menidia.............. 46

beryllinus, Acyrtops............... 48

beta, Opsanus. . . . . . . . . . . . . . 49

bicaudalis, Lactophrys. . . . . . . . . . . . . . 49

bicolor, Gila...................... 13

Siphateles................. 17

bicornis, Icelus. . . . . . . . . . . . . 39

bifasciatum, Thalassoma............ 34

bifrenatus, Notropis. . . . . . . . . . . . 15

biguttata, Hybopsis . . . . . . . . . . . 14

bilinearis, Merluccius............... 22

bilineata, Lepidopsetta . . . . . . . . . . . . 47 bilobus, Blcpsias............... 39

binoculata, Raja.............. 8

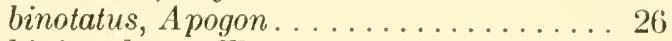

bipinnulatus, Elagatis............ 30

birostris, Manta.................. 9

bison, Enophrys. . . . . . . . . . . . . . 39

bivittatum, Diplectrum............ 25

bivittatus, Halichoeres. . . . . . . . . . . 33

blackfordi, Lutjanus. . . . . . . . . . . . . . 26

blanchardi, Neoclinus. . . . . . . . . . . 43

Blenniidae. . . . . . . . . . . . . . . . 43

blennioides, Etheostoma . . . . . . . . 27

Blennius...................... 43

blennius, Etheostoma............... 27

Notropis. . ................... 15

Blepsias....................... 39

Bodianus...................... 33

boekci, Callionymus............... 42

boleoides, Radulinus. . . . . . . . . . . . 40

Boleosoma-see Etheostoma

boleosoma, Gobionellus.............. 36

Bollmannia.................. 35

bonaci, Mycteroperca............. 25

bonariense, Haemulon. . . . . . . . . . . 30

bonasus, Rhinoptera.............. 9

boops, Notropis.................. 15

borealis, Icelinus. . . . . . . . . . . . . . 39

Sphyraena.................. 45

Boreogadus.................... 22

bosci, Gobiosoma................ 36

bosquianus, Chasmodes............. 43

Bothidae...................... 46

Bothragonus.................. 40

Bothus...................... 46

bovinus, Cyprinodon.............. 21

brachycephalus, Corythoichthys......... 23

brachychir, Bellator................ 38

Brachygenys.................. 30

Brachyistius................... 33

brachyptera, Remora............... 48

brachypterus, Parexocoetus........... 21

brachysomus, Calamus. . . . . . . . . . . 32

Brama..................... 30

Bramidae. . . . . . . . . . . . . . . . 30

Branchiostegidae............... 29

Brannerella................... 42

brasiliensis, Hemiramphus. . . . . . . . . 20

Narcine................... 8

Saurida.................... 13

Scorpaena................... 36

braytoni, Notropis................ 15

brazosensis, Notropis............... 15

brederi, Pseudogrammus............ . 25

breviceps, Moxostoma.............. 18

brevipes, Lycodes. . . . . . . . . . . . . 44

brevirostris, Chasmistes........... 17

Negaprion................ 7 
brevirostrum, Acipenser.

brevispinis, Sebastodes.

Brevoortia.

Brosme.

brosme, Brosme

Brosmophycis.

Brotula.

Brotulidae.

broussonneti, Gobioides

brucus, Echinorhinus. .

brunneus, Apristurus.

bubalus, Ictiobus

buccanella, Lutjanus.

buccata, Ericymba.

buccula, Notropis.

buchanani, Notropis

bullisi, Holocentrus.

burchami, Icelinus.

\section{C.}

caballus, Caranx...

Caccula.

caeruleofasciatus, Bathymaster

caeruleum, Etheostoma.

caeruleus, Notropis .

cahni, Hybopsis.

Calamus.

calamus, Calamus.

calcarata, Scorpaena

californica, Oxyjulis

Squatina

Torpedo

californicus, Cypselurus

Mustelus.

Myliobatis.

Paralichthys.

californiensis, Atherinopsis.

Medialuna.

Syngnathus.

Typhlogobius

Xenistius.

Callechelys.

Callionymidae

Callionymus

callisema, Notropis

callistius, Notropis.

callitaenia, Notropis

calliurus, Callionymus

Ioglossus.

callyodon, Liparis

calva, Amia

Calycilepidotus- see Hemilepidotus

campechanus, Lutjanus. .

Campostoma.

camurum, Etheostoma

camurus, Notropis.

canadense, Stizostedion. canadum, Rachycentron............ 29

canis, Mustelus............... 7

Cantherines.................... 48

Canthidermis.................. 48

Canthigaster..................... 49

capellei, Lophotus.................. 24

capistratus, Chaetodon.............. 32

caprinus, Stenotomus.............. 32

capriscus, Balistes................ 48

caprodes, Percina................ 29

Caproidae.................... 24

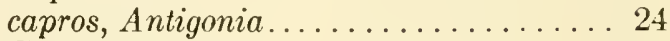

Carangidae................... 29

Caranx........................ 29

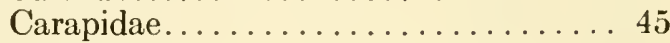

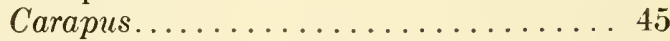

Carassius..................... 13

carbonarium, Haemulon. . . . . . . . . . 30

Carcharhinidae............... 7

Carcharhinus................. 7

Carcharias.................. 6

carcharias, Carcharodon........... 7

Carchariidae................. 6

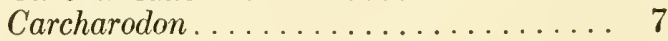

Careproctus...................4 41

caribbaea, Saurida.............. 13

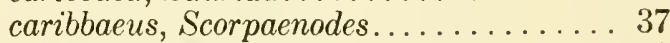

carinatum, Moxostoma............. 18

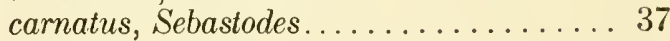

carolinae, Cottus................ 39

carolinensis, Balistes-see capriscus, $B$.

carolinus, Prionotus................. 38

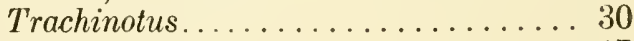

carpio, Carpiodes............... 17

Cyprinus................. 13

Floridichthys.................. 21

Carpiodes.................. 17

carri, Microgobius.............. 36

caryi, Hypsurus. . . . . . . . . . . . 33

castaneus, Ichthyomyzon.......... 6

castor, Pontinus................ 36

catalinensis, Rhynchocymba........ 19

cataractae, Rhinichthys............ 16

catenata, Echidna.................. 19

catenatus, Fundulus.............. 21

Catostomidae.................. 17

Catostomus.................... 17

catostomus, Catostomus............. 17

Phenacobius................. 16

catus, Ictalurus. . . . . . . . . . . . 18

caudalis, Halichoeres. . . . . . . . . . . 33

caudilimbatus, Conger............. 19

Caulolatilus.................... 29

caurinus, Mylocheilus............. 14

Sebastodes................. 37

cavalla, Scomberomorus............. 35

cavifrons, Ambloplites............ 27 
Icelinus.

39

cayorum, Anchoa

Ogilbia.

Cebidichthys

Centrarchidae.

Centrarchus.

Centriscidae.

Centrolophus.

Centropomidae

Centropomus

Centropristes

Centropyge.

Centroscyllium

centroura, Dasyatis .

cepedianum, Dorosoma

Cephalopholis

Cephaloscyllium

cephalus, Mugil.

cerasinus, Notropis

Ceratacanthus-see Alutera

cerdale, Scytalina.

ceriinum, Lepophidium.

Moxostoma.

Cetengraulis.

Cetorhinus.

ceuthoccus, Barbulifer

Chaenobryttus

Chaenopsis.

Chaetodipterus.

Chaetodon

chaetodon, Enneacanthus

Chaetodontidae

chalcogrammus, Theragra

chalybaeus, Notropis.

chamaeleonticeps, Lopholatilus.

Characidae.

Chascanopsetta .

Chasmistes.

Chasmodes.

Cheilotrema.

chesteri, Phycis.

chihuahua, Notropis

chiliensis, Sarda.

chiliticus, Notropis

Chilomycterus.

Chimaerae.

Chimaeridae.

Chimaeriformes

Chirolophus.

chirurgus, Acanthurus.

chirus, Phytichthys.

Chitonotus.

chittendeni, $C_{y}$ clopsetta .

Chlamydoselachidae.

Chlamydoselachus.

chloristius, Notropis.

chlorocephalus, Notropis
11

45

44

27

27

23

45

24

24

25

32

8
Chloroscombrus................. 29

chlorosomum, Etheostoma........... 27

chlorostictus, Sebastodes............. 37

Chologaster................... 22

Chondrichthyes................. 6

Chondrostei.................. 9

chordatus, Stylephorus............. 24

Chriodorus....................20 20

Chromis.................... 33

Chrosomus.................... 13

chrosomus, Notropis............... 15

chrysargyrea, Brachygenys. . . . . . . 30

chrysochloris, Alosa.............. 10

chrysogaster, Agosia............... 13

chrysomelas, Sebastodes............ 37

chrysops, Roccus................ 26

Stenotomus.................. 32

chrysopterum, Sparisoma.......... 34

chrysopterus, Orthopristis. . . . . . . . 31

chrysotus, Fundulus . . . . . . . . . . . 21

chrysura, Bairdiella.............. 31

chrysurus, Chloroscombrus.......... 29

Microspathodon............... 33

Ocyurus................... 26

chuss, Urophycis................... 22

Cichlasoma................... 33

Cichlidae.................... 33

ciliaris, Holacanthus. . . . . . . . . . . 32

ciliatus, Monacanthus.............. 49

Sebastodes................. 37

cimbrius, Enchelyopus............. 22

cinereum, Etheostoma. . . . . . . . . . 27

cinereus, Gerres. . . . . . . . . . . . . . . . 30

cingulatus, Fundulus.............. 21

Paraclinus................... 43

cirratum, Ginglymostoma.......... 7

cirratus, Urophycis. . . . . . . . . . . . 22

cirrhosus, Blepsias................ 39

Citharichthys................. 46

civitatus, Symphurus............. 48

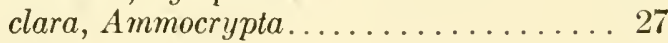

clarki, Pantosteus................. 18

Salmo..................... 11

clathratus, Paralabrax............ 25

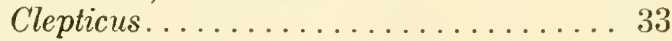

Clevelandia.................... 35

Clinidae........ . . . . . . . . . . . . 42

Clinocottus.................... 39

Clinostomus..................... 13

Clupea....................... 10

clupeaformis, Coregonus............ 11

Clupeidae.................... 10

Clupeiformes.................... 10

clupeola, Harengula. . . . . . . . . . . . . 10

Cobitidae. . . . ................. 18

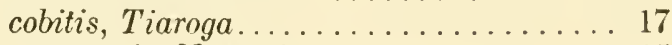

coccogenis, Notropis.............. 15 
coelestinus, Scarus.................. 34

coenosus, Pleuronichthys............ 47

coeruleus, Acanthurus............. 34

Scarus...................... 34

cognatus, Cottus................... 39

colburni, Seriola................. 30

colias, Scomber.................. 35

collapsum, Moxostoma............ 18

colliei, Hydrolagus. . . . . . . . . . . . 9

collis, Etheostoma.............. 27

Cololabis...................... 20

columbianus, Catostomus............. 17

comatus, Cypselurus............... 20

combatia, Antigonia............... 24

commersoni, Catostomus. ........... 17

compressa, Anchoa................ 11

conchorum, Menidia..............446

concolor, Scomberomorus............ 35

confluentus, Fundulus. . ........... 21

Conger........................ 19

congestum, Moxostoma........... 18

Congridae..................... 19

Congrina..................... 19

conklini, Apogon............... 26

C'aecula..................... 19

connectens, Lethops. . . . . . . . . . . . 36

conocephalus, Mylopharodon........ 14

Conodon..................... 30

constellatus, Sebastodes............. 37

coosae, Etheostoma.............. 27

Micropterus............... 27

copei, Snyderichthys............. 17

copelandi, Percina.............. 29

corallinum, Cryptotrema........... 42

corallinus, Artedius. . . . . . . . . . . . 38

Coregonus..................... 11

coregonus, Moxostoma.............. 18

coriacea, Moаpa................. 14

corinus, Hexanchus-see griseum, $H$.

cornuta, Chologaster.

cornutus, Citharichthys.

Notropis.

coroides, Umbrina.

corporalis, Semotilus.

cortczianus, Aprodon.

coruscus, Holocentrus.

Coryphaena.

Coryphaenidae $\ldots \ldots \ldots \ldots \ldots \ldots \ldots \ldots \ldots \ldots$

Coryphopterus................... 35

Corythoichthys.................. 23

Cottidae................... 38

Cottus....................... 39

Couesius..................... 14

coulteri, Prosopium............. 11

cragini, Etheostoma. . . . . . . . . . 27

crameri, Hybopsis. . . . . . . . . . 14

Sebastodes................... 37 crassa, Percina................... 29

crassicauda, Gila................. 13

creaseri, Artedius................ 39

Crenichthys.................. 21

crinigerus, Micrognathus........... 23

crinitus, Alectis. . .............. 29

Micrognathus................ 23

cristatus, Blennius.............. 43

Trachipterus............... 24

Cristivomer-see Salvelinus

crocro, Pomadasys................. 31

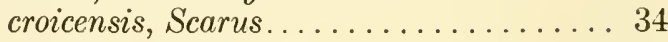

cromis, Pogonias. . . . . . . . . . . . . . . 31

crossotus, Etropus. . . . . . . . . . . . . 46

crotalina, Embryx.............. 44

cruentatum, Petrometopon............ 25

cruentatus, Priacanthus.............. 26

crumenophthalmus, Selar........... 30

Cryptacanthodes................. 44

Cryptopterygium................ 19

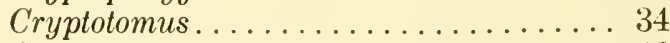

Cryptotrema................. 42

crysoleucas, Notenigonus............. 14

crysos, Caranx.................. 29

cubana, Anchoa.................... 11

cubensis, Squalus.................. \&

Cubiceps.................... 45

cujus, Chasmistes................. 17

cummingsae, Notropis............... 15

curacao, Bathygobius............. 35

curema, Mugil................ 46

cuvieri, Galeocerdo................. 7

Tetragonurus................ 45

cyanea, Chromis................. 33

cyanellus, Lepomis. . . . . . . . . . . 27

cyanoguttatum, Cichlasoma.......... 33

cyanophrys, Psenes............... 45

cyanops, Caulolatilus............... 29

cyanopterus, Cypselurus........... 20

Lutjanus................... 26

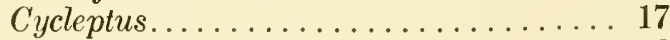

Cyclopsetta................. 46

Cyclopterichthys................ 41

Cyclopteridae...................4 41

Cyclopteropsis................. 41

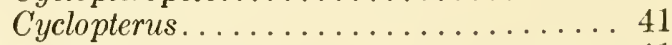

cyclopus, Liparis. . . . . . . . . . . . . . 41

cyclostigma, Liparis. . . . . . . . . . . . 4 41

cylindraceum, Prosopium............ 11

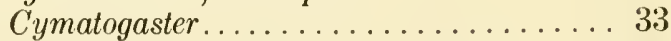

cymatotaenia, Percina............. 29

Cynoglossidae. ............... 48

cynoglossus, Glyptocephalus. . . . . . . . 47

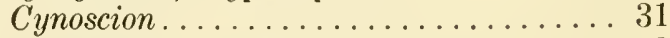

cypha, Gila................. 13

cyprinellus, Ictiobus. . . . . . . . . . . 17

Cyprinidae................. 13 
Cypriniformes

Cyprinodon

13

Cyprinodontidae

Cyprinodontiformes

cyprinoides, Lophogobius.

Cyprinus

cyprinus, Carpiodes.

Cypselurus.

\section{D}

Dactylopteridae

Dactylopterus .

dactylopterus, Helicolenus.

Dactyloscopidae.

Dactyloscopus.

Dalatias.

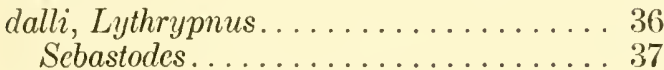

Dallia..................... 12

Damalichthys - see Rhacochilus

Dasyatidae.

Dasyatis. .

Dasycottus . .

davidsoni, Anisotremus.

deani, Paraliparis.

Polistotrema.

decagonus, Agonus.

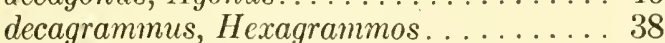

Decapterus. . . . . . . . . . . . . . . . 29

declivifrons, Vomer............... 30

Decodon.................... 33

decurrens, Pleuronichthys.......... 47

delicatissima, Anchoa ............ 11

Delolepis..................... 44

delphinus, Pantosteus............ 18

dennyi, Liparis. . . . . . . . . . . . . . 41

dentatus, Apsilus............... 26

Neoscopelarchoides............. 13

Paralichthys.................47 47

Pseudupeneus................. 31

dentex, Odontoscion............... 31

Osmerus...................... 12

denticulatus, Anarhichas.......... 43

derjugini, Eumicrotremus. .......... 41

Dermatolepis.................... 25

Dermatostethus................ 23

diaboli, Dionda................ 13

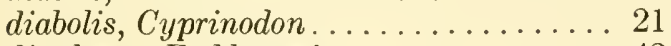

diaphana, Emblemaria . . . . . . . . . 42

Lactoria... . . . . . . . . . . . . . . . . 49

diaphanes, Raja-see ocellata, $R$.

diaphanus, Fundulus.

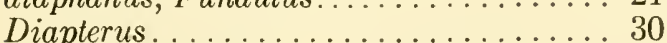

diapterus, Lycodes............... 44

dilatus, Spirinchus............... 12

dilecta, Ancylopsetta . ............ 46

Diodon.......................... 49
Diodontidae................. 49

diomedianus, Symphurus........... 48

Dionda.................... 13

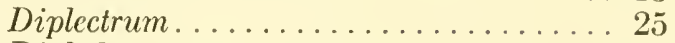

Diplodus...................... 32

diploproa, Scbastodes............. 37

dipterurus, Dasyatis. . . . . . . . . . . 9

Discocephali.................... 48

dispar, Scorpaena............... 36

dissimilis, Hybopsis.............. 14

ditaenia, Gila................ 14

ditropis, Lamna................ 7

dodecaedra, Occa..............440

dolichogaster, Pholis............. 43

dolomieui, Micropterus............. 27

Doratonotus..................... 33

Dormitator. . . . . . . . . . . . . 35

dormitor, Gobiomorus.............. 35

Dorosoma..................... 10

dorsalis, Notropis................ 15

Seriola.................... 30

Sphaeroides................. 49

dorsopunicans, Eupomacentrus. . . . . . . 33

drummondhayi, Epinephelus........ 25

dubius, Ammodytes............... 41

ductor, Naucrates................ 30

dumerili, Seriola................. 30

Squatina................... 8

dunckeri, Syngnathus. . . . . . . . . 23

duodecim, Anchoa ............... 11

duquesnei, Moxostoma.............. 18

duryi, Etheostoma............. 27

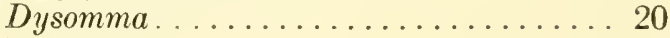

Dysommidae................. 20

E

earlli, Urophycis. . .............. 22

Echeneidae.................. 48

Echeneiformes................. 48

Echeneis....................... 48

Echidna..................... 19

Echinorhinus................ 8

edwini, Etheostoma............. 28

eglanteria, Raja............... 8

egmontis, Ahlia............... 19

egregius, Arioscopus............. 42

Richardsonius................. 17

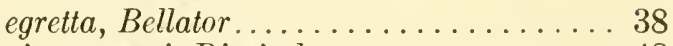

eigenmanni, Rimicola............. 48

Elacatinus................... 35

Elagatis................... 30

elassodon, Hippoglossoides. . . . . . . . 47

Elassoma..................... 27

elater, Zalieutes................ 50

elegans, Cyprinodon............. 21

Gibbonsia................. 42

Eleginus................... 22 
Eleotridae..................... 35

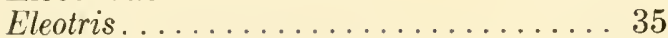

eleutherus, Noturus. . . . . . . . . . . . 18

ellipticum, Hyperprosopon . . . . . . . . . 33

elongatus, Allosmerus............. 12

Clinostomus................. 13

Cycleptus................. 17

Ophiodon..................... 38

Sebastodes................. 37

Elopidae..................... 10

Elops....................... 10

elucens, Syngnathus.............. 23

Embassichthys................. 47

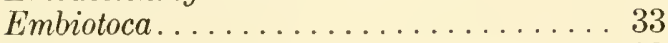

Embiotocidae................... 33

Emblemaria..................... 42

embryum, Clinocottus............. 39

Embryx.................... 44

emiliae, Opsopoeodus.............. 16

emmelane, Agonopsis............. 40

Empetrichthys................ 21

emphaeus, Sebastodes.............. 37

Enchelycore................ 19

Enchelyopus.................. 22

enchrysurus, Chromis............ 33

Engraulidae................. 11

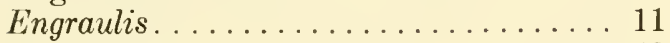

Engyophrys................ 46

Enneacanthus.................. 27

Enneapterygius................. 42

Enophrys.................... 39

ensiferus, Centropomus . . . . . . . . . 24

Entomacrodus................. 43

entomelas, Sebastodes............. 37

Entosphenus-see Lampetra

Eopsetta...................... 47

eos, Chrosomus................. 13

Sebastodes.................. 37

Ephippidae.................. 32

Epinephelus.................. 25

episcopa, Dionda . . . . . . . . . . . . 13

Eques-see Equetus

Equetus..................... 31

equisetis, Coryphaena. . . . . . . . . 30

erectus, Hippocampus. . . . . . . . . . 23

Eremichthys................ 13

Ericymba................. 13

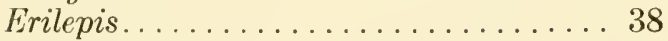

Erimystax................. 14

Erimyzon.................... 17

erinacea, Raja............... 8

Erotelis. ...................... 35

erythra, Gibbonsia.............. 42

erythrogaster, Chrosomus........... 13

erythrophthalmus, Scardinius......... 17

crythrurum, Moxostoma............ 18

Esocidae..................... 12
Esox.................... 12

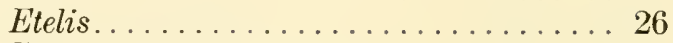

Etheostoma.................. 27

etowanum, Hypentelium. . . . . . . . . . 17

Etropus..................... 46

Etrumeus..................... 10

Eucalia........................ 23

Eucinostomus................. 30

Eucyclogobius................. 35

Euleptorhamphus............... 20

Eumesogrammus................ 44

Eumicrotremus................. 41

Eupomacentrus................. 33

eurystole, Anchoviella.............. 11

eurystomus, Satan................ 19

euryzonus, Notropis............... 15

Euthynnus.................. 34

euzonum, Etheostoma............ 28

evergladei, Elassoma. . . . . . . . . . 27

evermanni, Rhinichthys............ 16

Evermannichthys ............. 36

evides, Percina................ 29

Plectobranchus................... 44

Eviota . . . . . . . . . . . . . 35

evolans, Prionotus. . . . . . . . . . . . 38

Evorthodus.................... 36

exasperata, Zapteryx............. 8

exile, Etheostoma. . . . . . . . . . . . . . 28

exilicauda, Lavinia . . . . . . . . . . . . . 14

exilis, Lyopsetta. . . . . . . . . . . . 47

Noturus.................... 18

Strongylura................ 20

Exocoetidae.................. 20

Exocoetus. . . . . . . . . . . . . . . . . . 21

Exoglossum.................... 13

exsiliens, Cypselurus. . . . . . . . . . 20

extensa, Menidia................ 46

Extrarius...................... 14

\section{F}

faber, Chaetodipterus............ 32

fabricii, Centroscyllium........... 8

Lumpenus. . . . . . . . . . . . . . . . . 44

falcata, Seriola................ 30

falcatus, Rhinichthys............ 16

Trachinotus................ 30

falciformis, Carcharhinus.......... 7

fasciata, Pholis................. 43

Seriola.................... 30

fasciatus, Larimus. . . . . . . . . . . . 31

Opisthognathus.............. 41

Paraclinus.................... 43

fecundus, Catostomus.............. 17

felis, Galeichthys................ 18

fenestralis, Artedius.............. 39

ferox, Alepisaurus............... 13

ferruginea, Limanda............. 47 
fierasfer, Lycodapus.

filamentosus, Icelinus.

fimbria, Anoplopoma.

fimbriata, Cyclopsetta.

fimbriatus, Icelinus

Fistularia.

Fistulariidae.

flabellare, Ethcostoma.

flammea, Hemitremia

flava, Congrina.

flavescens, Perca.

flavidus, A podichthys.

Aulorhynchus

Sebastodes.

flavobrunneum, Lepidocybium

flavolimbatus, Epinephelus.

flavolineatum, Haemulon.

flavus, Noturus.

florae, Liparis.

floridae, Jordanella

Syngnathus.

floridanus, Carcharhinus.

Microdesmus.

Urophycis. .

Floridichthys.

focaliger, Menticirrhus.

Fodiator.

foetens, Synodus.

fonticola, Etheostoma

fontinalis, Salvelinus.

forbesi, Carpiodes.

forcipatus, Balistes.

formosa, Heterandria

Mollienesia.

formosum, Diplectrum

fossor, Ichthyomyzon.

francisci, Heterodontus

freminvillei, Myliobatis .

frenata, Zaniolepis.

frenatus, Brachyistius. .

Sarritor.

fricksium, Etheostoma

frontalis, Gastropsetta

fryi, Hesperomyrus

fucensis, Liparis.

fucorum, Xererpes.

fulgida, Meda.

fulva, Cephalopholis.

fulvescens, Acipenser

fumeus, Notropis.

funduloides, Clinostomus

Fundulus.

funebris, Gymnothorax.

Noturus

furcatus, Cypselurus

Ictalurus.

Phanerodon furcifer, Paranthias............. 25

furiosus, Noturus. . . . . . . . . . . . 18

fuscus, Syngnathus............... 23

fusiforme, Etheostoma ............. 28

\section{G}

Gadidae

Gadiformes.................... 22

Gadus...................... . 22

gagei, Ichthyomyzon............ 6

gaigei, Gambusia . . . . . . . . . . . . . . . . . 22

gaimardiana, Mugil.............. 46

gairdneri, Salmo................. 11

galacturus, Notropis.............. 15

Galeichthys................... 18

Galeocerdo.................... 7

Galeorhinus................... 7

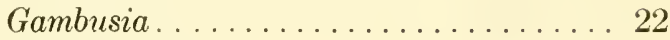

garmani, Raja.............. 8

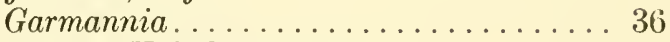

garnoti, Halichoeres. ............. 33

Garrupa-see Epinephelus

Gasterosteidae... . . . . . . . . . . . . . . 23

Gasterosteiformes................ 23

Gasterosteus................... 23

Gastropsetta................ 47

geiseri, Gambusia............... . 22

gelida, Hybopsis................ 14

geminatus, Hypleurochilus. . . . . . . . . 43

gemmiferum, Prosopium............ 11

Gempylidae.................... 34

Gempylus..................... 34

gentilis, Hypsoblennius. . . . . . . . . . 43

Genyonemus . . ................ 31

Germo--see Thunnus

Gerres........................ . 30

Gerridae................... 30

gibbifrons, Prognichthys. .......... 21

Gibbonsia.................... 42

gibbosus, Lepomis. . . . . . . . . . . . . . . 27

gigantea, Delolepis............... 44

gigas, Stereolepis. . . . . . . . . . . . 26

Gila........................ 13

gilae, Salmo................. 11

gilberti, Hypsoblennius . . . . . . . . . . 43

Ilypnus.................. . 36

Noturus................... 18

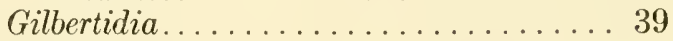

Gillellus.................... 42

gilli, Pholis................ 43

Sebastodes.................... 37

Synchirus................. 40

Gillichthys.................. 36

Ginglymodi . . . . . . . . . . . . . . 10

Ginglymostoma.............. 7

ginsburgi, Gobiosoma. . . . . . . . . . . 36

girardi, Notropis . . . . . . . . . . . 15 
Girella.

Girellidae.

griseus, Lutjanus. .

glaber, Cyclopterichthys............ 41

glacialis, Arctogadus. . . . . . . . . . . . 22

Liopsetta. . . . . . . . . . . . . . 47

gladius, Xiphias............... 35

glauca, Prionace............... 7

glaucofraenum, Coryphopterus........ . 35

glaucum, Stizostedion vitreum......... 29

glaucus, Isurus................ 7

Trachinotus................. 30

glesne, Regalecus. . . . . . . . . . . . . 24

globiceps, Clinocottus.............. 39

gloriosus, Enneacanthus. . . . . . . . . 27

glutinosa, Myxine............... 6

Glyptocephalus................ 47

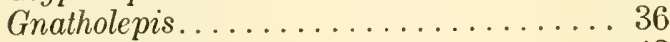

Gobiesocidae................ 48

Gobiesociformes............... . 48

Gobiesox................... 48

Gobiidae... . . . . . . . . . . . . . . 35

Gobioides.................... 36

gobioides, Bembrops............. 42

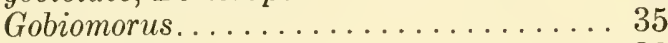

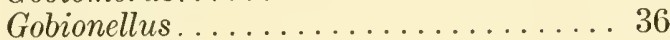

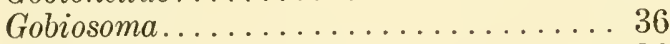

Gobulus.................. 36

gomesi, Ophichthus.............. 20

Gonioplectrus.................. 25

goodei, Lucania . . . . . . . . . . . . . . 22

Myliobatis................ 9

Ptilichthys.................. 44

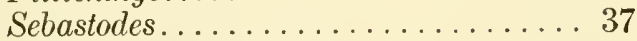

gorbuscha, Oncorhynchus.......... 11

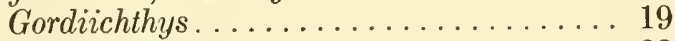

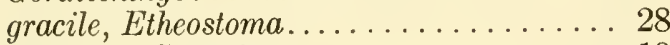

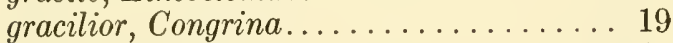

gracilis, Cubiceps................ 45

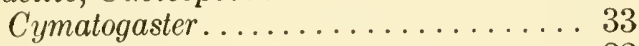

Eleginus.................... . . 22

Hybopsis.................... 14

Macrorhamphosus............. 23

gracillimus, Gobionellus............. 36

graellsi, Lepophidium............. 45

grahami, Etheostoma.............. 28

grandicomis, Paraclinus............. 43

grandicornis, Scorpaena . . . . . . . . . 36

grandis, Fundulus............... 21

Ptychocheilus............... 16

grceleyi, Ichthyomyzon........... 6

greenei, Cottus.................. 39

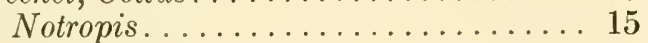

greeni, Polypera............... 41

greyae, Gillellus. . . . . . . . . . . . 42

greyi, Istiophorus. . . . . . . . . . 35

griseolineatus, Syngnathus. . . . . . . . 23

griseum, Hexanchus.............. 6

gronowi, Nomeus................. 45

grunniens, Aplodinotus............. 31

guacamaia, Scarus................ 34

guachancho, Sphyraena............ 46

gula, Eucinostomus. . . . . . . . . . . . 30

gulosus, Chaenobryttus............ 27

Cottus...................... 39

Microgobius............... 36

gunnellus, Pholis.............. 43

gunteri, Brevoortia . . . . . . . . . . . . 10

Syacium.................. 47

guppyi, Labrisomus. . . . . . . . . . . 43

gutselli, Etheostoma............ 28

guttata, Scorpaena............... 37

guttatus, Astroscopus. . . . . . . . . . . 42

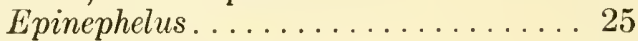

guttulata, Hypsopsetta. . . . . . . . . 47

Gymnachirus................ 47

Gymnelis................... 44

Gymnocanthus................ 39

Gymnothorax................. 19

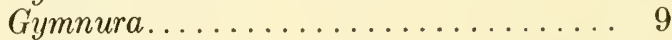

gyrinus, Noturus................ 18

Gyropleurodus-see Heterodontus

H

Haemulon....................... 30

Halecomorphi . . . . . . . . . . . . . . 10

Halichoeres.................... 33

Halieutichthys.................. 50

halleri, Urolophus. . . . . . . . . . ... 9

hamiltoni, Manta................. 9

Moxostoma..................... 18

hankinsoni, Artedius. . . . . . . . . . 39

Hybognathus . .............. 14

Haplodoci...................... 49

Harengula.................... 10

harengus, Clupea harengus.......... 10

harperi, Hybopsis................ 14

harringtonensis, Allanetta.......... 46

harringtoni, Artcdius............... 39

hastatus, Gobionellus............... 36

havana, Eucinostomus.............. 30

hayi, Hybognathus................ 14

heidi, Uraspis. . . . . . . . . . . . . . . . 30

Helicolenus..................... 36

helvomaculatus, Sebastodes........... 37

Hemanthias..................... 25

Hemibranchii ................... 23

Hemicaranx. . . . . . . . . . . . . . 30

Hemiemblemaria................. 42

Hemilepidotus.................... 39

hemilepidotus, Hemilepidotus.......... 39

Hemiramphidae.................. 20

Hemiramphus................... 20

Hemitremia................... 14 
Hemitripterus.

hemphilli, Stathmonotus

henlei, Triakis.

hentzi, Hypsoblennius

hepsetus, Anchoa

Herichthys - see Cichlasoma

Hermosilla .

Hesperoleucus.

Hesperomyrus. .

Heterandria.

Heteristius.

heterochir, Gambusia.............. 22

heteroclitus, Fundulus.............. 21

heterodon, Notropis.

Heterodontidae.

Heterodontus.

heterolepis, Notropis

Heteromi

Heterosomata.

Heterostichus.

heterurus, Cypselurus.

Hexagrammidae.

Hexagrammos.

Hexanchidae.

Hexanchus.

hexapterus, Ammodytes

hians, Ablennes.

hildebrandi, Noturus

Hiodon

Hiodontidae.

Hippocampus

Hippoglossina .

Hippoglossoides.

hippoglossoides, Reinhardtius

Hippoglossus .

hippoglossus, Hippoglossus.

hippos, Caranx.

hippurus, Coryphaena

Hirundichthys.

hirundo, Leiocottus.

hispanus, Gonioplectrus

hispidus, Monacanthus

Histrio

histrio, Etheostoma.

Histrio. .

Holacanthus.

holacanthus, Diodon

holbrooki, Diplodus

Ophidion.

Holconotus-see Amphistichus

holderi, Alloclinus.

Holocentridae .

Holocentrus.

holochroma, Cryptopterygium.

hopkinsi, Plagiogrammus.

Sebastodes.

hopliticus, Paricelinus.
39
Hoplunnis................. 19

horsti, Gobiosoma............... 36

hoyi, Coregonus.................. 11

hubbsi, Cottus................... 39

Cyprinodon................. 21

Ichthyomyzon................ 6

Moxostoma................. 18

Novumbra. . . . . . . . . . . . . . . . 12

hudsonius, Notropis............... 15

humeralis, Harengula . . . . . . . . . . 10

humilis, Lepomis. . . . . . . . . . . . . . . 27

Hybognathus.................. 14

Hybopsis..................... 14

Hyborhynchus - see Pimephales

Hydrolagus.................... 9

Hypentelium.................. 17

Hyperoartii. . . . ............ 6

Hyperotreti. . . . . . . . . . . . . . 6

Hyperprosopon. . . . . . . . . . . . . 33

Hypleurochilus................ 43

hypochromus, Allolumpenus......... 44

hypodus, Decapterus. . . . . . . . . . . . . 29

Hypomesus. . . . . . . . . . . . . . . . . 12

Hypoplectrus................... 25

hypoplectus, Rathbunella........... 42

Hypoprion................. 7

Hyporhamphus................... 20

hypostoma, Mobula.............. 9

Hypsagonus . . . . . . . . . . . . . 40

hypselopterus, Notropis............ 15

hypsilepis, Notropis............... 15

hypsinotus, Hybopsis............... 14

Hypsoblennius. ............... 43

Hypsopsetta . ...............44 47

Hypsurus. . ................ 33

Hypsypops................ 33

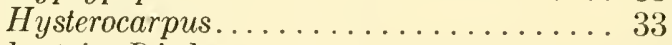

hystrix, Diodon............... 49

\section{I}

Icelinus. . . . . . . . . . . . . . . 39

Icelus..................... 39

Ichthyomyzon.............. 6

Icichthys................. 45

icistius, Bairdiella............. 31

Icosteidae. . . . . . . . . . . . . . . . . 45

Icosteus. . . . . . . . . . . . . 45

Ictaluridae................. 18

Ictalurus. . . . . . . . . . . . . . . . 18

Icticus. . . . . . . . . . . . . 45

Ictiobus. . . . . . . . . . . . . . 17

illecebrosus, Notropis.............. 15

Ilypnus.................. 36

imberbe, Peristedion.............. 38

imperialis, Luvarus. . . . . . . . . . . . 35

impressa, Ariosoma.............. 19

incisor, Kyphosus. . . .......... 32 
inconstans, Eucalia

indica, Makaira

inermis, Anoplagonus

Dermatolepis.

Scorpaena.

infraspinata, Asterotheca.

ingens, Hippocampus.

Iniomi. .

inornata, Raja

inscriptum, Etheostoma

inscriptus, Trinectes.

insignis, Catostomus .

Hybopsis.

Noturus.

insolatus, Chromis

integripinnis, Paraclinus

intermedius, Pantosteus.

Synodus.

interrupta, Raja.

interruptus, Archoplites.

interstitialis, Mycteroperca

intertinctus, Mystriophis.

Ioglossus.

ionthas, Hypsoblennius.

ios, Clevelandia.

Iotichthys.

irretitus, Gordiichthys .

isodon, A prionodon . .

isolepis, Isopsetta

Isopsetta .

Isospondyli.

Istiophoridae

Istiophorus.

Isurus.

itajara, Epinephelus

\section{J}

jacksoni, Embiotoca.

jacobus, Myripristis

jamaicensis, Urolophus.

japanica, Mobula.

japonica, Lampetra .

japonicus, Arctoscopus

Scomber

jeannae, Bollmannia.

Lepophidium

jemczanus, Notropis.

jenkinsi, Fundulus.

Jenkinsia

jessiae, Etheostoma

jocu, Lutjanus.

johannae, Coregonus

Jordanella.

jordani, Eopsetta

Etheostoma.

Mycteroperca .

Ronquilus.
Sebastodes. 37

Jordania..................... . 39

juliae, Etheostoma.............. 28

\section{$\mathbf{K}$}

kalisherae, Labrisomus. . . . . . . . . . . . 43

kanawhae, Etheostoma............. 28

kansae, Fundulus................ 21

Kathetostoma................. 42

Katsuwonus-see Euthynnus

kendalli, Verma............... 20

kennicotti, Etheostoma........... 28

keta, Oncorhynchus.............. 11

kincaidi, Malacocottus.............40

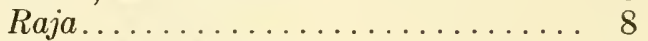

kisutch, Oncorhynchus............ 11

kiyi, Coregonus................... 11

klamathensis, Cottus.............. 39

koefoedi, Liparis. . . . . . . . . . . . 41

koclzi, Amphistichus.............. 33

Kyphosidae................. 32

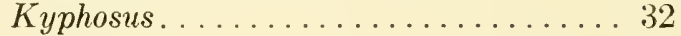

\section{$\mathbf{L}$}

Labidesthes. . . . . . . . . . . . . . . . 46

Labridae. . . . . . . . . . . . . . . . . . . 33

Labrisomus. . . . . . . . . . . . . . . . . . 43

labrosa, Hybopsis. . . . . . . . . . . 14

lacera, Lagochila . . . . . . . . . . . . . 17

lachneri, A pogon. . . . . . . . . . . . 26

Moxostoma.............. 18

Lachnolaimus. . . . . . . . . . . . . . . 34

lachrymale, Moxostoma............ 18

Lactophrys . . . . . . . . . . . . . . . 49

Lactoria. . . . . . . . . . . . . . . . . 49

lacustris, Ictalurus-see punctatus, $I$.

laeta, Pholis................... 43

laevigatus, Lagocephalus . . . . . . . . . . 49

laevis, Raja................. 8

Ranzania................... 49

Lagocephalus . . . . . . . . . . . . . . . . 49

lagocephalus, Lagocephalus. . . . . . . . . 49

Lagochila . . . . . . . . . . . . . . . 17

Lagodon..................... . 32

lahontan, Pantosteus. . . . . . . . . 18

lamiella, Carcharhinus. ........... 7

Lamna... . . . . . . . . . . . . . . . . . 7

Lamnidae.................... 6

lamottei, Lampetra. . . . . . . . . . . . . 6

Lampetra................... 6

Lamprididae.................. 23

Lampridiformes. . . . . . . . . . . . . . 23

Lampris. . . . . . . . . . . . . . . . 23

lamprotaenia, Anchoa........... 11

Jenkinsia.................... 10

lanccolata, Mola................ 49

lanceolatus, Equetus.............. 31 
lanceolatus, Stellifer

Larimus.

lateralis, Artedius

Embiotoca

lathami, Trachurus

latifrons, Xeneretmus.

latipinna, Mollienesia.

latipinnis, Catostomus.

Zaniolepis.

latos, Empetrichthys.

latus, Caranx.

laurae, Parexoglossum.

lavalaci, Lycodes.

Lavinia .

leedsi, Notropis.

lefroyi, Eucinostomus

Leiocottus.

leiopomus, Cottus.

leiops, Xeneretmus.

Leiostomus.

lentiginosa, Raja.

lentiginosus, Rhinobatos

Lepibema-see Roccus

Lepidocybium.

Lepidogobius.

Lepidomeda.

Lepidopsetta. .

Lepidopus

lepidum, Etheostoma

lepidus, Lepidogobius .

Notropis.

Lepisosteidae.

Lepisosteus.

Lepomis.

Lepophidium.

leptacanthus, Noturus

leptocephala, Hybopsis

Leptocottus.

Leptolucania.

leptorhynchus, Sarritor

lepturus, Trichiurus.

leptus, Hemanthias .

Letharchus.

Lethops.

lethostigma, Paralichthys

leucas, Carcharhinus .

Leucichthys .

leucichthys, Stenodus.

leuciodus, Notropis.

leucosteus, Calamus.

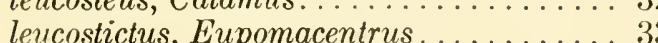

Leuresthes.

Leuroglossus. . . . . . . . . . . . . . . . . . . 12

levis, Sebastodes. . . . . . . . . . . . . 37

lewini, Sphyrna.............. 7

libertate, Opisthonema . . . . . . . . 10

licha, Dalatias .
Limanda

limbatus, Carcharhinus............... 7

limi, Umbra. ................ 12

lindneri, Lonchopisthus. . . . . . . . . . . 41

lineata, Parahollardia . . . . . . . . . 48

lineatus, Achirus............... 47

Euthynnus.................. 34

Genyonemus................. 31

Oostethus................... 23

Phtheirichthys................ 48

lineopunctatus, Xanthichthys........ 49

liolepis, Xystreurys............ 47

Liopsetta. . . . . . . . . . . . . . 47

liorus, Chasmistes. . . . . . . . . . . 17

Liparis. . . . . . . . . . . . . . . . 41

liparis, Liparis . . . . . . . . . . . . . 41

lirus, Notropis................. 15

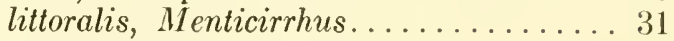

liza, Mugil. . . . . . . . . . . . . . . 46

Lobotes... . . . . . . . . . . . . . . . 26

Lobotidae. . . . . . . . . . . . . . 26

lockingtoni, Icichthys. . . . . . . . . 45

Lonchopisthus. . . . . . . . . . . . . . 41

lonchurus, Opisthognathus. . . . . . . . . 41

longicaudus, Gobionellus . . . . . . . . . 36

longimanum, Etheostoma. . . . . . . . . . . . 28

longimanus, Carcharhinus. . . . . . . . 7

longipala, Gobiosoma............. . 36

longipinnis, Careproctus........... 41

Microdesmus............... 36

Taractes................... 30

longirostris, Lumpenella. . . . . . . . . 44

Notropis................. 15

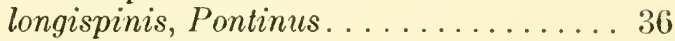

longum, Gobiosoma. . . . . . . . . . . 36

longurio, Scoliodon.............. 7

Lophiidae. . . . . . . . . . . . . . . . . 49

Lophiiformes . . . . . . . . . . . . . . . . 49

Lophius. . . . . . . . . . . . . . . . . . 49

Lophobranchii. . . . . . . . . . . . . . 23

Lophogobius . . . . . . . . . . . . . . . 36

Lopholatilus. . . . . . . . . . . . . . 29

Lophopsetta-see Scophthalmus

Lophotidae. . . . . . . . . . . . . . . . . 24

Lophotus. . . . . . . . . . . . . . . . 24

Lota. . . . . . . . . . . . . . . . . . . 22

lota, Lota.................... 22

louisianae, Syngnathus........... 23

lowei, Polymixia . . . . . . . . . . . . 24

Lucania....................... 22

lucasana, Mobula............... 9

luciac, Fundulus. . . . . . . . . . . 21

lucioceps, Synodus. . . . . . . . . . . . 13

lucius, Esox.................. 12

Ptychocheilus............. 16

lugubris, Caranx. . . . . . . . . . . . 29

Chascanopsetta.............. 46 
Lumpenella..................... 44

Lumpenus..................... 44

lumpretaeformis, Lumpenus. . . . . . . . . 44

lumpus, Cyclopterus. . . . . . . . . . . . 41

lunatus, Bothus. . ............. 46

lunulatus, Mustelus.............. 7

iupus, Anarhichas..............44 43

Ictalurus. . . . . . . . . . . . . . . 18

luteovinctum, Etheostoma. . . . . . . . 28

lutipinnis, Notropis.............. 15

Lutjanidae. . . . . . . . . . . . 26

Lutjanus................... 26

lutrensis, Notropis. . . . . . . . . . 15

Luvaridae. . . . . . . . . . . . . . . . . 35

Luvarus. . . . . . . . . . . . . . . . 35

luxatus, Catostomus............. 17

Lycenchelys.................... 44

Lycichthys-see Anarhichas

Lycodapus. . . . . . . . . . . . . . . 44

Lycodes . . . . . . . . . . . . . . . . . . . 44

Lycodopsis. . . . . . . . . . . . . . . . . 44

Lyconectes. . . . . . . . . . . . . . . 44

Lyconema. . . . . . . . . . . . . . 44

lyolepis, Anchoa............... 11

Lyopsetta. . . . . . . . . . . . . . 47

lyricus, Evorthodus. . . . . . . . . . 36

Lythrypnus. . . . . . . . . . . . . 36

M

macalpini, Cyclopteropsis .

macarellus, Decaptcrus. . . . . . . . . . . 29

macdonaldi, Scbastodes............. 37

maccllus, Triglops............... 40

Macrhybopsis ................. 14

macrocephala, Percina............ 29

macrocephalus, Gadus............... 22

macrocheilus, Catostomus.......... 17

macrochirus, Lepomis. . . . . . . . . . 27

macrodon, Garmannia............. 36

macrognathus, Opisthognathus........ 41

macrolepidotum, Moxostoma......... 18

macrolepidotus, Pogonichthys........ 16

Macropinna................. 12

macrops, Citharichthys............ 46

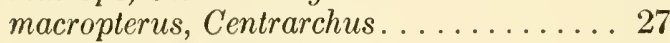

macropus, Malacoctenus. . . . . . . . . . 43

Macrorhamphosus............... 23

macrostomum, Hacmulon. . . . . . . . 31

Macrouridae................. 22

Macrozoarces................ 44

macrurus, Hoplunnis. . . . . . . . . . . 19

macularius, Cyprinodon........... 21

maculata, Percina. . . . . . . . . . . . 29

maculatofasciatus, Paralabrax......... 25

maculatum, Etheostoma............ 28

maculatus, Apogon............. 26

Aulostomus................... 23
Canthidermis............... 48

Cryptacanthodes.............. 44

Dormitator. . . . . . . . . . . . . . 35

Lumpenus................... 44

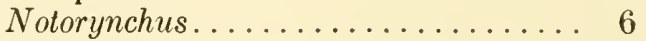

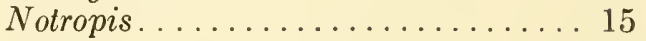

Psenes....................4.45

Pseudupeneus............... 32

Scomberomorus............... 35

Sphaeroides................ 49

Trinectes................. 47

maculipinna, Halichoeres........... 34

maculipinnis, Carcharhinus......... 7

maculosus, Oligocottus. . . . . . . . . . 40

maeandricus, Gobiesox............. 48

mahogoni, Lutjanus.............. 26

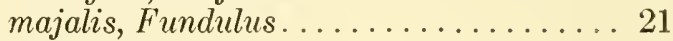

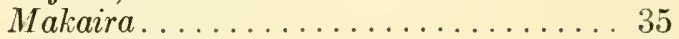

Malacanthus................... 29

Malacocottus............... 40

Malacoctenus................ 43

maliger, Scbastodes.............. 37

Mallotus.................. 12

malma, Salvelinus.............. 12

mandibularis, Lycodapus. . . . . . . . . 44

Manta.................... 9

margarita, Semotilus............. 17

marginata, Brosmophycis.......... 45

Rissola.................. 45

marginatus, Cottus.............. 39

Lepomis. . . . . . . . . . . . . . . 27

mariac, Etheostoma.............. 28

marianus, Holocentrus. . . . . . . . . . 24

marina, Strongylura............. 20

marinus, Bagre. . . . . . . . . . . . 18

Petromyzon............... 6

Sebastes................... 37

marmorata, Gymnura............ 9

marmoratus, Paraclinus. . . . . . . . . 43

Rivulus. ................. 22

Scorpaenichthys.............. 40

marmoreus, Blennius.............. 43

martinica, Membras............. 46

martinicensis, Ocyanthias........... 25

Xyrichthys................ 34

martinicus, Mulloidichthys.......... 31

martis, Prionotus. . . . . . . . . . . . . 38

masquinongy, Esox. . . . . . . . . 12

matutinus, Notropis............... 15

maxillingua, Exoglossum . . . . . . . . 13

maxillosus, Opisthognathus. . . . . . . . . 41

maximus, Cetorhinus.............. 7

Lachnolaimus................. 34

megintyi, Ogcocephalus. . . . . . . . . . . 50

meanyi, Ruscarius. .............. 40

Meda....................... 14

Medialuna................. 32 
mediocris, Alosa

medirostris, Acipenser

medius, Lumpenus.

meeki, Hybopsis.

megalepis, Doratonotus.

Megalops.

megalotis, Lepomis

Melanogrammus.

melanops, Minytrema.

Scbastodes

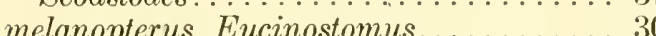

melanostictus, Psettichthys. . . . . . . . . 47

melanostomus, Sebastodes.

mclanurum, Haemulon.

melanus, Centropristes.

melas, Ictalurus. .

Melichthys.

Membras.

Menidia.

menidia, Menidia.

Menticirrhus.

Merluccius.

merriami, Empetrichthys.

Mesogonistius - see Enneacanthus

metzi, Gibbonsia.

mexicana, Pikea. .

mexicanus, Astyanax...

miarcha, Anchoviella.

microccphalus, Somniosus.

Microcyprini.

Mierodesmidae.

Microdesmus.

microdon, Pseudotriakis .

Microgadus.

Micrognathus

Microgobius.

microlepidotus, Orthodon .......... 16

microlepis, Microgobius............ 36

Mycteroperca...............25

Scorpaena................. 37

microlophus, Lepomis . . . . . . . . . . . 27

Micrometrus.................. 33

microperca, Etheostoma........... 28

Micropogon................. 31

micropogon, Hybopsis. . . . . . . . 14

microps, Catostomus. . . ......... 17

Micropterus................ 27

micropterus, Oxyporhamphus........ 21

Microspathodon................ 33

microstoma, Macropinna............ 12

Microstomus.................. 47

microstomus, Etropus. . . . . . . . . 46

micrura, Gymnura............ 9

micrurum, Syacium............. 47

milberti, Carcharhinus. ........... 7

miliaris, Muraena.............. 19

militaris, Bellator............... 38 miniatum, Peristedion............. 38

miniatus, Sebastodes.............. 37

minimus, Micrometrus............ 33

minor, Anarhichas................ 43

Symphurus................. 48

Minytrema................ 17

mirabilis, Gillichthys............ 36

Phenacobius................. 16

Misgurnus............... 18

mississippiensis, Roccus........... 26

mitchilli, Anchoa.............. 11

miurus, Noturus. . . . . . . . . . . . . 19

Moapa.................... 14

Mobula...................9

Mobulidae.................... 9

mohavensis, Siphatcles.............. 17

mokarran, Sphyrna.............. 7

Mola.................... 49

mola, Mola................ 49

Molidae....................... 49

Mollienesia.................... 22

Monacanthus................... 49

monacha, Hybopsis. . . . . . . . . . . 14

monoceros, Alutera................. 48

Monolene..................... 47

monopterygius, Aspidophoroides....... 40

Pleurogrammus............... 38

montereycnsis, Gibbonsia........... 42

monticola, Agonostomus. ........... 46

mordax, Engraulis................ 11

Gymnothorax............... 19

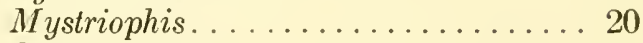

Osmerus.................. 12

morhua, Gadus................. 22

moringa, Gymnothorax. . . . . . . . 19

morio, Epincphelus. . . . . . . . . . . 25

Morone-see Roccus

Moxostoma................. 17

mucosus, Liparis. . . . . . . . . . . . . . . 41

Xiphister................. 44

mucronatus, Neoconger. . . . . . . . . 19

Mugil...................... 46

Mugilidae................... 46

Mullidae...................... 31

Mulloidichthys................. 31

Mullus...................... 31

multilineata, Chromis............. 33

multiocellatus, Antennarius......... 50

Muraena....................... 19

muraena, Callechelys............... 19

Muraenidae.................... 19

muscarum, Rimicola............ 48

Mustelus.................... 7

Mycteroperca................ 25

Myctophidae................ 13

Myctophiformes.............. 13

myersi, Gobulus............... 36 
Myliobatidae

Myliobatis.

Mylocheilus. .

Mylopharodon................... 14

myops, Trachinocephalus........... 13

Myoxocephalus................ 40

myriaster, Porichthys.............. 49

Myrichthys.................. 19

Myripristis.................. 24

Myrophis................... 20

mystacinus, Epinephelus............ 25

Opisthognathus................ 42

mysticetus, Cetengraulis............ 11

mystinus, Sebastodes. . . . . . . . . . . 37

Mystriophis.................. 20

Myxine..................... 6

Myxinidae................... 6

Myxiniformes .

Myxinoidea .

\section{$\mathbf{N}$}

namaycush, Salvelinus .

Narcine

narinari, Aetobatus.

nasus, Coregonus.

Lamna.

Notacanthus .

nasuta, Percina

nasutus, Ogcocephalus................ 50

natalis, Ictalurus. . . . . . . . . . . 18

Naucrates..................... 30

naucrates, Echeneis.............. 48

Nautichthys................. 40

nebulifer, Paralabrax............. 25

nebulosus, Cynoscion............... 31

Ictalurus.................. 18

Sebastodes.................. 37

Nectoliparis. . ................... 41

Negaprion.................. 7

Nematistius................... 30

Nemichthyidae.................. 19

Neoclinus..................... 43

Neoconger..................... 19

neogaeus, Chrosomus............... 13

Neomerinthe................... 36

Neoscopelarchoides............... 13

Neothunnus-see Thunnus

nephelus, Sphaeroides.

nerka, Oncorhynchus

neucratoides, Echeneis.

nevadae, Crenichthys. .

nevadensis, Cyprinodon

newberryi, Eucyclogobius.

Nezumia.

nianguae, Ethcostoma .

nicholsi, Blennius.

Coryphopterus.
Nicholsina................... 34

niger, Centrolophus.............. 45

Esox................... 12

Ictiobus.................... 17

nigrescens, Gila............... 14

nigriargenteus, Cubiceps........... 45

nigricans, Enchelycore............. 19

Girella..................... 32

Hypentelium................ 17

Makaira................... 35

nigricinctus, Labrisomus............ 43

nigripinnis, Bathyagonus.......... 40

Coregonus.................... 11

Paraclinus.................. 43

nigritus, Epinephelus............. 25

nigrocinctus, Sebastodes.............. 37

nigrofasciata, Percina.............. 29

nigromaculatus, Pomoxis........... 27

nigromarginatus, Gymnothorax....... 19

nigrum, Etheostoma............. 28

nipigon, Coregonus.............. 11

nitens, Trichiurus.............. 34

niveatus, Epinephelus.............. 25

niveus, Notropis................. 15

nobiliana, Torpedo............... 8

nobilis, Conodon.................. 30

Cynoscion.................. 31

Gambusia.................... 22

Nocomis..................... 14

nocturnus, Noturus. . ............. 19

Nomeus...................... 45

normani, Saurida.............. 13

norrisi, Mustelus............... 7

Notacanthidae................ 20

Notacanthiformes. . . . . . . . . . . . . 20

Notacanthus................. 20

notata, Strongylura. . . . . . . . . . 20

notatus, Fundulus. . ............. 21

Pimephales.................. 16

Porichthys.................. 49

Notemigonus................... 14

nothus, Cynoscion................ 31

notius, Micropterus.............. 27

notogramma, Percina.............. 29

Notorynchus................... 6

notospilotus, Artedius............ 39

notospilus, Prionodes............... 25

Notropis..................... 14

notti, Fundulus. . . ............ 21

Noturus...................... 18

novaculum, Ophidion.............. 45

Novumbra..................... 12

nubila, Dionda................. 13

nuchalis, Hybognathus.............. 14

nuchipinnis, Labrisomus............. 43

nudus, Gymnachirus. ............. 47

nugator, Chirolophus.............. 44

nybelini, Triglops............... 40 
O

obesus, Enneacanthus

Thunnus.

obeyense, Etheostoma

oblongus, Erimyzon.

Paralichthys.

obscurus, Carcharhinus

obtusirostris, Exocoetus

obtusus, Hippocampus.

Occa

occidentalis, Catostomus

Poeciliopsis.

oceanicus, Conger.

Gobionellus...

oceanops, Elacatinus

ocellata, Brannerella.

Chaenopsis.

Raja

Sciaenops

Zenopsis

ocellatus, Anarrhichthys

Antennarius.

Astronotus.

Bothus.

Chaetodon

Ophichthus

octodecemspinosus, Myoxocephalus.

octogrammus, Hexagrammos.

octonemus, Polydactylus.

oculatus, Etelis

Icelinus.

Lepisosteus.

Myrichthys

oculofasciatus, Nautichthys.

Ocyanthias.

Ocyurus.

ocyurus, Centropristes.

Odontaspis-see Carcharias

Odontopyxis.

Odontoscion.

ogac, Gadus.

Ogcocephalidae

Ogcocephalus.

Ogilbia...

oglinum, Opisthonema

ohiensis, Alosa.

okefenokee, Elassoma...

olidus, Hypomesus.

Oligocottus

Oligoplites.

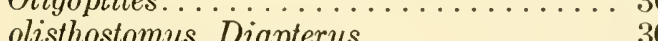

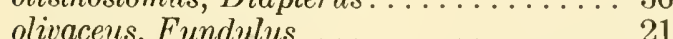

olivaris, Pylodictis. . . . . . . . . . . . . 19

olriki, Aspidophoroides. . . . . . . . . . . 40

olseni, Raja. . . . . . . . . . . . . 8

omiscomaycus, Percopsis........... 24

ommata, Leptolucania . . . . . . . . . . . . 22

omostigmum, Otophidium.
Oncorhynchus.

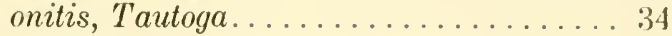

Oostethus..................... 23

opercularis, Polydactylus. . . . . . . . . 46

Ophichthidae. . . . . . . . . . . . . 19

Ophichthus..................20.

Ophidiidae................... 45

Ophidion................... 45

Ophioblennius................. 43

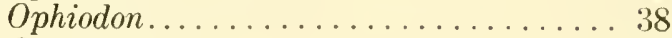

Ophioscion.................... 31

ophis, Ophichthus.............. 20

ophryas, Prionotus............... 38

Opisthognathidae............... 41

Opisthognathus..................41

Opisthonema.................. 10

Opisthoproctidae................ 12

Opsanus..................... 49

Opsopoeodus................. 16

orbis, Eumicrotremus.............. 41

orcutti, Gila. . . ............... 14

oreas, Chrosomus................. 13

Orectolobidae................. 7

oregonensis, Ptychocheilus........... 16

Oregonichthys.................. 14

orientalis, Anarhichas............. 43

Sarda.................... 35

ornata, Pholis................ 43

ornatum, Campostoma............ 13

ortenburgeri, Notropis. . . . . . . . . 15

Orthodon.................. 16

Orthonopias.................. 40

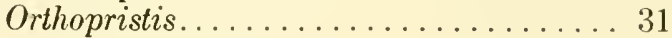

osburni, Etheostoma............... 28

osculus, Rhinichthys. . . . . . . . . . . 16

Osmeridae................... 12

Osmerus.................... 12

osseus, Lepisosteus. . . . . . . . . . . . . 10

Ostariophysi................. 18

Osteichthyes................. 9

osteochir, Remora............... 48

Ostichthys................... 24

Ostraciidae.................. 49

Otophidium................. 45

Otrynter-see Stenotomus

ovalis, Sebastodes................ 37

Oxyjulis.................... 34

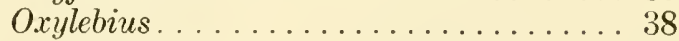

Oxyporhamphus................21

oxyrhyncha, Percina. . . . . . . . . . . 29

oxyrhynchus, Acipenser............ 9

Notropis.................... 15

oxyrinchus, Isurus. . . . . . . . . . 7

ozarcanus, Notropis............ 15

$\mathbf{P}$

pachycephalus, Lagocephalus. . . . . . . 49

pacifica, Lycodopsis. . . . . . . . . . . . 44 
pacificus, Microstomus.

Somniosus...

Thaleichthys.

\section{2}

Pagrus.

paitensis, Trachinotus.

palearis, Lycodes.

Palinurichthys.

pallasi, Clupea harengus

Pallasina

pallidus, Lycodes.

palmaris, Percina.

Palometa.

pandionis, Emblemaria.

pantherina, Percina.

Pantosteus.

papillifer, Gobiesox.

papillosum, Syacium

pappillosum, Moxostoma

Paracentropristes

Paraclinus.

paradoxus, Psychrolutes

Parahollardia.

Paralabrax

paralatus, Prionotus.

Paralichthys.

Paraliparis.

parallelus, Centropomus .

Paranthias.

Parathunnus-see Thunnus

pardus, Opsanus.

Parexocoetus.

Parexoglossum

Paricelinus.

Parmaturus.

parmifera, Raja

Parophrys.

parrai, Clepticus

Haemulon.

partitus, Eupomacentrus.

paru, Peprilus. . .

parva, Lucania. .

parvipinne, Etheostoma

parvipinnis, Cynoscion.

Fundulus.

parvus, Ogcocephalus.

Symphurus.

Upeneus.

patronus, Brevoortia.

pattersoni, Trogloglanis

paucispinis, Sebastodes.

pectinatus, Centropomus .

Pristis.

pectoralis, Dallia.

Enneapterygius.

Nematistius.

Prionotus.

Pediculati. pelagicus, Nectoliparis .

Syngnathus.

41

pelamis, Euthynnus.............. 34

pelicanus, Symphurus............. 48

pellucida, Ammocrypta............ 27

pellucidus, Icticus. . . . . . . . . . . . 45

peltata, Percina................ 29

Pempheridae.................. 32

Pempheris.................... 32

penna, Calamus................. 32

pensacolae, Harengula............. 10

pentacanthus, Asterotheca........... 40

Peprilus................... 45

Perca..................... 29

Percidae..................... 27

Perciformes.................. 24

perciformis, Palinurichthys. . . . . . . 45

Percina..................... 29

percobromus, Notropis............ 15

Percomorphi................. 24

Percophididae.................. 42

Percopsidae.................. 24

Percopsiformes................ 24

Percopsis..................... 24

perfasciata, Anchoviella............. 11

Peristedion.................. 38

perlongum, Etheostoma........... 28

perotteti, Pristis............... 8

perpallidus, Notropis............. 15

perplexus, Cottus................. 39

perryae, Callechelys............... 19

personata, Eviota............... 35

peruanus, Hemanthias. . . . . . . . . . . 25

petenense, Dorosoma . . . . . . . . . . . 10

petersoni, Notropis.............. 16

Petrometopon................. 25

Petromyzon................. 6

Petromyzontia.............. 6

Petromyzontidae............... 6

Petromyzontiformes............ 6

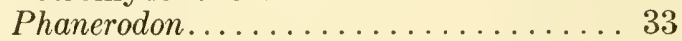

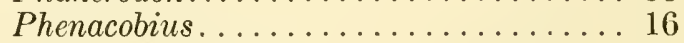

phenax, Mycteroperca............ 25

philadelphicus, Centropristes......... 25

phlegethontis, Iotichthys. . . . . . . . 14

phoebe, Prionodes. . . . . . . . . . . . . 25

Pholidae................... 43

Pholis...................... 43

photogenis, Notropis. . ......... 16

phoxocephala, Percina............ 29

Phtheirichthys.................. 48

Phycis.................... 22

Phytichthys............... 44

picta, Eleotris. . . . . . . . . . . 35

pictus, Oxylebius. . . . . . . . . . . . 38

picudilla, Sphyraena............. 46

pidschian, Coregonus.............. 11 
piger, Symphurus.

pigmentarius, Apogon

Pikea.

pilicornis, Blennius

Pimelometopon.

Pimephales

pingeli, Triglops.

pinniger, Sebastodes.

piratula, Emblemaria.

pisonis, Eleotris.

Placopharynx - see Moxostoma

Plagiogrammus

plagiusa, Symphurus.

Plagopterus.

planeri, Lampetra

planifrons, Apogon.

Eupomacentrus.

platessoides, Hippoglossoides.

Platichthys.

platorynchus, Scaphirhynchus

platostomus, Lepisosteus

platycephalus, Ictalurus

Platygobio

platyrhincus, Lepisosteus.

Platyrhinoidis.

platyrhynchus, Pantosteus.

plebeius, Pantosteus.

Plectobranchus.

Plectognathi.

Plectrypops.

Pleurogrammus.

Pleuronectes. .

Pleuronectidae.

Pleuronectiformes

Pleuronichthys.

plumbea, Hybopsis.

plumieri, Diapterus.

Hacmulon.

Malacanthus.

Scorpaena

Pneumatophorus-see Scomber

podostemone, Etheostoma.

Poecilichthys - see Etheostoma

Poeciliidae.

Poeciliopsis.

poecilurum, Moxostoma.

poeyi, Halichoeres.

Synodus.

Pogonias.

Pogonichthys.

Polistotrema.

politus, Seriphus.

Pollachius.

pollux, Neomerinthe.

polyacanthocephalus, Myoxocephalus.

polyactocephalus, Chirolophus.

Polydactylus.
48 polylepis, Balistes

26

25

43

34

16

40

37

42

35

44

48

16

Polymixia.................... 24

Polymixiidae................. 24

Polynemidae................ 46

Polyodon...................... 9

Polyodontidae................ 9

Polypera................... 41

Polyprion....................25

polyspinis, Scbastodes............. 37

polystictus, Trachipterus............ 24

Pomacanthus.................. 32

Pomacentridae.... . . . . . . . . . . . . . 33

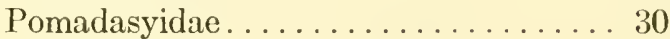

Pomadasys..................... 31

Pomatomidae................... 29

Pomatomus...................... 29

Pomolobus - see Alosa

pomospilus, Paracentropristes........ 25

pomotis, Acantharchus............. 27

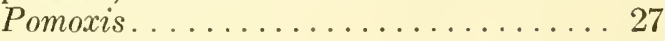

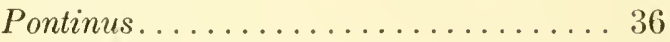

Porichthys.................. 49

Poroclinus.................. 44

Poronotus...................... 45

porosissimus, Porichthys............ 49

porosus, Carcharhinus............. 7

potteri, Notropis............... 16

praecisus, Eumesogrammus. . . . . . . . 44

pretiosus, Hypomesus. . . . . . . . . . . . 12

Ruvettus................. 34

Priacanthidae............... 26

Priacanthus................. 26

pricei, Ictalurus. . . . . . . . . . . . 18

princeps, Caulolatilus.............. 29

Cottus..................... 39

Prionace.................... 7

Prionodes.................... 25

Prionotus..................... 38

Pristidae................. 8

Pristigenys.................. 26

Pristipomoides................ 26

Pristis.................... 8

probatocephalus, Archosargus......... 32

proboscidea, Limanda. . . . . . . . . . 47

procne, Notropis................ 16

productus, Merluccius.............. 22

Rhinobatos................. 8

proeliare, Etheostoma. . . . . . . . . . 28

Prognathodes.................. 32

Prognichthys................... 21

promelas, Pimephales. . . . . . . . . . 16

Promicrops-see Epinephelus

Pronotogrammus............... 25

proridens, Calamus............... 32

proriger, Sebastodes. . . . . . . . . . . 37

proserpinus, Notropis............. 16

Prosopium................. 11 
Protospondyli................ 10

proximus, Microgadus.............. 22

Psenes.................... 45

Psettichthys............... 47

Pseudogrammus................ 25

pseudogula, Eucinostomus. . . . . . . . 30

pseudoharengus, Alosa............ 10

pseudomaculatus, Apogon.......... 26

Pseudopleuronectes.............. 47

Pseudoscarus - see Scarus

Pseudotriakis................ 7

Pseudupeneus.................. 31

psittacus, Xyrichthys.............. 34

Psychrolutes.................. 40

Ptilichthyidae............... 44

Ptilichthys................... 44

Ptychocheilus................ 16

puellaris, Decodon.............. 33

pugetensis, Chitonotus............. 39

pulchellus, Bodianus............. 33

Liparis. . . . . . . . . . . . . . . 41

pulcher, Equetus................ 31

pulchrum, Pimelometopon........... 34

pullus, Cantherines............... 48

pulvereus, Fundulus.............. 21

pumilio, Serraniculus............. 26

punctatus, Decapterus. . . . . . . . . . . 29

Equetus................... 31

Ictalurus. . . . . . . . . . . . 18

Lepomis.................. 27

Myrophis.................. 20

Stichaeus................... 44

punctifer, Mystriophis............ 20

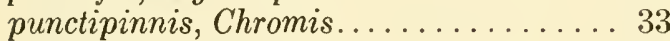

Dermatostethus................. 23

punctulatum, Etheostoma........... 28

punctulatus, Gobiesox. ............ 48

Micropterus................ 27

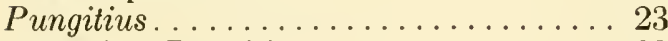

pungitius, Pungitius............ 23

purpurea, Gila................. 14

purpurescens, Anoplarchus........... 44

pusillus, Symphurus.............. 48

putnami, Liopsetta . . . . . . . . . . . . . 47

pygmaea, Umbra . . . . . . . . . . . 12

Pylodictis.................. 19

pyrrhomclas, Notropis............ 16

Q

quadracus, Apeltes.............. 23

quadricornis, Hypsagonus. . . . . . . . 40

Lactophrys . ................... 49

Myoxocephalus.............. 40

quadriseriatus, Icelinus. . . . . . . . . . . . 39

quadrisquamatus, Apogon........... 26

quadrituberculatus, Pleuronectes........ 47

quadrocellata, Ancylopsctta..........446

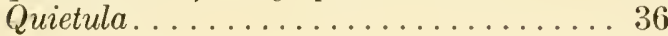

$\mathbf{R}$

Rachycentridae............... 29

Rachycentron.................. 29

radians, Sparisoma.............. 34

radiata, Raja. .............. 8

radiatus, Halichoeres. . . . . . . . . . . . 34

Ogcocephalus................. 50

radiosum, Etheostoma . . . . . . . . . 28

radiosus, Antennarius............. 50

Cyprinodon.................. 21

radula, Melichthys...............448

Radulinus.................. 40

Raja..................... 8

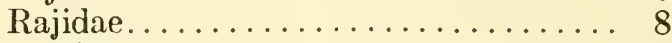

Rajiformes................. 8

randalli, Acanthurus............. 34

Ranzania.................. 49

raphidoma, Strongylura. . . . . . . . . 20

rastrelliger, Sebastodes............. 37

Rathbunella................ 42

rathbuni, Fundulus.............. 21

Pontinus................... 36

rayi, Brama................ 30

recalvus, Clinocottus.............. 39

Regalecidae.................. 24

Regalecus................... 24

regalis, Cynoscion.............. 31

Scomberomorus................ 35

regius, Lampris . . . . . . . . . . . . 23

Urophycis................. 22

regulus, Psenes............... 45

reighardi, Coregonus. . . . . . . . . . 11

Reinhardtius................ 47

Remora................... 48

remora, Remora............... 48

Remorina................. 48

remotus, Carcharhinus............. 7

reticulatus, Lycodes. . . . . . . . . . . 44

retifer, Scyliorhinus............. 7

retifera, Muraena................. 19

retrospinis, Plectrypops........... 24

rex, Percina................. 29

Rhacochilus.................. 33

Rhamphocottus...............440

rhessodon, Gobiesox............. 48

rhina, Raja................ 8

Rhincodon................ 7

Rhincodontidae............. 7

Rhinichthys............... 16

Rhinobatidae............... 8

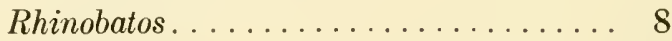

Rhinoptera................. 9

Rhodeus................... 16

rhodochloris, Sebastodes............ 37

rhodopus, Trachinotus. . . . . . . . . . 30

rhodorus, Ascelichthys............. 39

rhodoterus, Amphistichus............ 33

rhomboidalis, Archosargus.......... 32 
rhomboides, Lagodon

Rhomboplites.

rhotheus, Cottus.

rhothoecum, Moxostoma

Rhynchocymba

ricei, Cottus

richardsoni, Alepisaurus

Rhamphocottus.

Richardsonius.

rimensis, Oligocottus

Rimicola

rimiculus, Catostomus

rimosus, Etropus.

ringens, Xanthichthys.

Risor.

Rissola

ritteri, Pleuronichthys .

Xeneretmus.

Rivulus

roanokense, Hypentelium

robusta, Gila.

robustum, Gobiosoma

Moxostoma.

robustus, Hippoglossoides

Roccus.

Roncador.

roncador, Umbrina

rondeleti, Prognichthys

Ronquilus

rosaceus, Sebastodes.

Zalembius

rosae, Amblyopsis.

Hyporhamphus.

roseipinnis, Notropis.

roseus, Cryptotomus.

Prionotus.

rosispinis, Raja.

rostrata, Anguilla

Canthigaster.

rostratus, Heterostichus

rothrocki, Poroclinus.

rubellio, Oligocottus.

rubellus, Notropis.

ruber, Caranx.

Risor.

ruberrimus, Sebastodes

rubicunda, Hypsypops .

rubio, Prionotus.

rubreques, Moxostoma-see valenciennesi, $M$.

rubricroceus, Notropis

rubrifrons, Hybopsis.

rubripinne, Sparisoma

rubrivinctus, Sebastodes.

rubrocinctus, Heteristius

rubrofluviatilis, Cyprinodon

rufilineatum, Etheostoma.

rufus, Bodianus.
32

26

39

18

19

39

13

40

17

40

48

17

47

49

36

45

47

41

22

17

14

36

18

47

25

31

31

21

42

37

33

22

20

16

34

38
Holocentrus................. 24

rupestre, Etheostoma............ 28

rupestris, Ambloplites............. 27

rupiscartes, Moxostoma........... 18

Ruscarius................... 40

rutteri, Liparis . . . . . . . . . . . . . 41

Ruvettus...................... 34

Rypticus................ 26

\section{$\mathbf{S}$}

sabina, Dasyatis............... 9

sabinae, Notropis................ 16

saburrae, Chasmodes.............. 43

sadina, Etrumeus................ 10

sagax, Sardinops............... 10

sagitta, Etheostoma . . . . . . . . . . 28

Lumpenus................... 44

saida, Boreogadus. . . . . . . . . . . . 22

saira, Cololabis.................. 20

salar, Salmo.................. 11

salinus, Cyprinodon.............. 21

Salmo....................... 11

salmoides, Micropterus. ........... 27

Salmonidae................... 11

Salmopercae.................. 24

saltator, Hemiramphus. . . . . . . . . 20

saltatrix, Pomatomus.............. 29

saludae, Etheostoma . . . . . . . . . . 28

Salvelinus..................... 12

sanctaerosae, Ulvicola............ 43

santaanae, Pantosteus. . . . . . . . . . . 18

sapidissima, Alosa............. 10

saponaceus, Rypticus............. 26

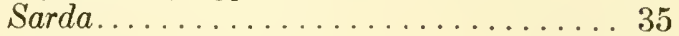

sarda, Sarda.................. 35

Sardinella................. 10

sardinella, Coregonus.............. 11

Sardinops................... 10

Sarritor..................... 40

Satan..................... 19

saturnum, Cheilotrema............ 31

Saurida..................... 13

saurus, Elops. ................ 10

Oligoplites. . . . . . . . . . . . . 30

Scomberesox................. 20

saxatilis, Abudefduf............... 33

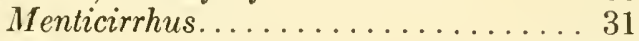

Roccus.................. 26

saxicola, Sebastodes............. 37

sayanus, Aphredoderus............. 24

sayi, Dasyatis................... 9

scaber, Antennarius............. 50

scabrata, Raja-see radiata, $R$.

scabriceps, Notropis............... 16

Scaphirhynchus................. 9

Scardinius.................. 17

Scaridae. . . . . . . . . . . . . . . . 34

Scarus....................... 34 
scepticus, Notropis.

16

Schilbeodes-see Noturus

schoepfi, Alutera.

Chilomycterus.

schomburgki, Pempheris

schultzi, Pholis.

sciadicus, Fundulus. .

Sciaenidae.

Sciaenops.

sciera, Percina.

scitulus, Prionotus.

sciurus, Haemulon.

Scoliodon.

scolopax, Macrorhamphosus............ 23

Scomber...................... 35

Scomberesocidae................ 20

Scomberesox................. 20

Scomberomorus................. 35

Scombridae.................. 34

scombrus, Scomber............... 35

Scopelarchidae................ 13

Scophthalmus................. 47

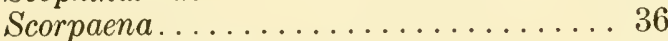

Scorpaenichthys............... 40

Scorpaenidae................. 36

Scorpaenodes................. 37

Scorpidae. . . . . . . . . . . . . . . . . . 32

scorpioides, Myoxocephalus. . . . . . . . 40

scorpius, Myoxocephalus............ 40

scovelli, Syngnathus.............. 23

scrippsae, Otophidium............ 45

scripta, Alutera................ 48

scuticaris, Bascanichthys.......... 19

Scyliorhinidae.

Scyliorhinus.

Scytalina.

Scytalinidae.

Sebastes.

Sebastodes.

Sebastolobus.

sectatrix, Kyphosus

secundodorsalis, Thunnus-see thynnus, $T$.

sedecim, Pagrus................. 32

sedentarius, Chaetodon............ 32

Selachii.

Selar

Selene.

selenops, Ophidion

sellare, Etheostoma

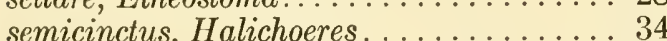

Sebastodes.

semifasciata, Triakis

seminolis, Fundulus.

Semionotiformes .

Semotilus.

senilis, Gambusia. senta, Raja.

sentus, Engyophrys. . . . . . . . . . 46

sericeus, Rhodeus................ 16

Seriola..................... 30

Seriphus.................... 31

serpens, Gempylus............... 34

Serranellus.................. 26

Serraniculus................. 26

Serranidae.................. 25

serranoides, Sebastodes............. 38

Serranus.................... 26

serriceps, Sebastodes.............. 38

serriferum, Etheostoma. . . . . . . . . . 28

serrula, Pristigenys. . . . . . . . . . . 26

sessilicauda, Monolene. . . . . . . . . . 47

setapinnis, Vomer.............. 30

setifer, Monacanthus............. 49

setiger, Dasycottus . . . . . . . . . . . . 39

shufeldti, Gobionellus.............. 36

shumardi, Percina............. 29

sialis, Argentina . ............ 12

Vacuoqua................. 31

sicculus, Labidesthes . . . . . . . . . . . 46

sigalutes, Gilbertidia. . . . . . . . . . 39

signatus, Bathymaster. . . . . . . . . . . 42

Hypoprion. . ............. 7

signipinnis, Notropis. . ......... 16

silenus, Zaprora.............. 45

silus, Argentina . . . . . . . . . . . . 12

similis, Fundulus................ 21

simillima, Palometa............. 45

simoterum, Etheostoma........... 28

simulus, Hemiemblemaria........... 42

simus, Notropis. . . . . . . . . . . . 16

Siphateles................. 17

smaragdus, Erotelis. . . . . . . . . . . 35

Gobionellus................... 36

smithi, Brevoortia............. 10

snyderi, Catostomus. . . . . . . . . . . 17

Oligocottus.................. 40

Snyderichthys............... 17

solanderi, Acanthocybium.......... 34

Soleidae. . . . . . . . . . . . . . . . 47

Solenichthyes................ 23

Somniosus.................. 8

soporator, Bathygobius............ 35

sordidus, Citharichthys. . . . . . . . . 46

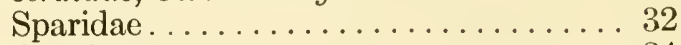

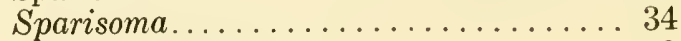

spathula, Polyodon............... 9

spatula, Icelus . . . . . . . . . . . . . . 39

Lepisosteus. . . . . . . . . . . . . . 10

spectabile, Etheostoma............ 28

spectrunculus, Notropis............ 16

spelaea, Amblyopsis.............. 22

spengleri, Sphaeroides............. 49

Sphaeroides.................. 49 
Sphyraena

Sphyraenidae

Sphyrna...

Sphyrnidae.

spilonotus, Prosopium.

spilopterus, Citharichthys

Notropis

spinicauda, Raja.

spinosa, Acanthemblemaria

spinosus, Eumicrotremus .

Hemilepidotus.

Spirinchus.

spongicola, Evermannichthys .

springeri, Gordiichthys .

Syngnathus.

Squalidae.

Squaliformes

Squalus

squamata, Percina.

squamiceps, Etheostoma

squamilentus, Paralichthys

Squatina

Squatinidae.

stabuliforis, Raja-see laevis, $R$.

stahli, Stathmonotus.

starksi, Spirinchus.

Stathmonotus.

stearnsi, Prionotus.

Roncador.

Stelgis

stellatus, Apogon

Platichthys.

stelleri, Hexagrammos.

Stcllerina.

Stellifer.

stellifer, Fundulus

stellulata, Raja.

Stenodus.

Stenotomus

Stephanolepis-see Monacanthus

stephanophrys, Prionotus .

stephensae, Neoclinus

Stereolepis.

sterletus, Agonopsis

Stichaeidae.

Stichaeus. .

stigmaeum, Etheostoma

stigmaeus, Citharichthys

stigmaticus, Gobionellus.

stigmaturus, Gobionellus.

stilbius, Leuroglossus.

Notropis.

stipes, Atherinomorus

Stizostedion.

stomata, Hippoglossina.

stomias, Atheresthes. storeriana, Hybopsis

stouti, Polistotrema

stramineus, Notropis............. 16

striatus, Centropristes. . . . . . . . . . 25

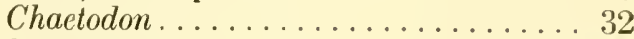

Epinephelus................ 25

Stromateidae................. 45

Strongylura.................. 20

strumosus, Gobiesox. . . . . . . . . . . . . 48

Stylephoridae................ 24

Stylephorus................... 24

subbifrenatus, Rypticus............ 26

subbifurcata, Ulvaria.............. 44

subligarius, Serranellus............. 26

subterraneus, Typhlichthys. . . . . . . . 22

sucetta, Erimyzon. . . . . . . . . . . . 17

sucklcyi, Squalus-see acanthias, S.

sufflamen, Canthidermis.

superciliosus, Alopias.

Hexagrammos.

surinamensis, Anisotremus.......... 30

Lobotes. . . . . . . . . . . . . . . . . 26

swaini, Etheostoma............. 28

swani, Bothragonus.............. 40

swannanoa, Etheostoma............. 28

Syacium..................... 47

symmetricus, Hesperoleucus. . . . . . . . 14

Lepomis. . . . . . . . . . . . . . . . 27

Trachurus.................. 30

Symphurus................. 48

synagris, Lutjanus. . . . . . . . . . 26

syncheilus, Catostomus-see columbianus, C.

Synchirus.

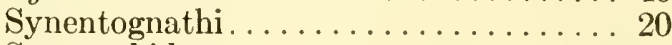

Syngnathidae. . . . . . . . . . . . . 23

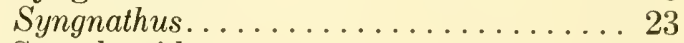

Synodontidae................. 13

Synodus...................... 13

synodus, Synodus................ 13

\section{$\mathbf{T}$}

tabacaria, Fistularia............ 23

Taeniotoca-see Embiotoca

tahoensis, Catostomus. . . . . . . . . . 17

tajasica, Awaous. . . . . . . . . . . 35

Taractes.................... 30

Tarpon-see Megalops

tau, Opsanus . . . . . . . . . . . . . . . 49

taurina, Enophrys. . . . . . . . . . . . . 39

taurus, Abudefduf ............... 33

Carcharias................. 6

Tautoga.................... 34

Tautogolabrus.................. 34

taylori, Asemichthys.............. 39

Otophidium............... 45

tenellus, Pimephales. . . . . . . . . . 16 
tenuis, Cottus 39

Erimyzon

Icelinus. .

Leuresthes

Urophycis.

teres, Bascanichthys

teretulus, Phenacobius.

tergisus, Hiodon.

terraenovae, Eumicrotremus

Scoliodon

testudineus, Sphaeroides

Tetragonuridae.

Tetragonurus.

Tetranarce-see Torpedo

Tetraodontidae.

Tetraodontiformes

Tetrapturus.

tetrazonum, Etheostoma.

texana, Raja

texanus, Notropis.

$X$ yrauchen.

textilis, Entomacrodus.

thalassinum, Etheostoma.

thalassinus, Microgobius.

Thalassoma.

Thaleichthys .............. 12

thaleichthys, Spirinchus

thazard, Auxis .

Theragra

thompsoni, Gnatholepis.

Ophioscion

Thoracostei.

thrissina, Harengula

Thunnus.

Thymallus.

thynnus, Thunnus.

Tiaroga.

tiburo, Sphyrna.

tigrinus, Myrichthys

tigris, Mycteroperca.

Isurus-see oxyrinchus, $I$.

timucu, Strongylura.

Tinca.

tinca, Tinca.

tippecanoe, Etheostoma.

tomcod, Microgadus.

topeka, Notropis.

Torpedinidae

Torpedo.

tortugarum, Serranus.

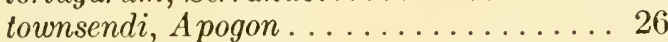

toxotes, Rhacochilus............. 33

Trachinocephalus.............. 13

Trachinotus................. 30

Trachipteridae............... 24

Trachipterus................. 24

trachura, Raja.

Trachurops-see Selar
Trachurus................ 30

trachypomus, Ostichthys........... 24

trachypterus, Trachipterus. . . . . . . . . 24

transmontana, Percopsis............ 24

transmontanus, Acipenser............ 9

traski, Hysterocarpus. . . . . . . . . . 33

treculi, Micropterus. . . .......... 27

Triacanthodidae.............. 48

triacanthus, Poronotus. . . . . . . . . . 45

Xeneretmus................41

triacis, Orthonopias. . . . . . . . . . 40

Triakis.................... 7

triangulatus, Malacoctenus.......... 43

tribulus, Prionotus................ 38

Trichiuridae................. 34

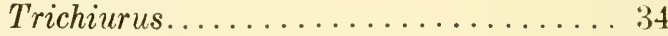

Trichodon..................41

trichodon, Mugil. ..............4 46

Trichodon.................41

Trichodontidae.............. 41

Trichopsetta................ 47

trichroistius, Notropis. . . . . . . . . 16

tricolor, Holacanthus. . . . . . . . . . . 32

tricuspis, Gymnocanthus............ 39

tridentata, Lampetra. . . . . . . . . . . . 6

tridigitatus, Dactyloscopus. . . . . . . . . 42

Triglidae..................... 38

Triglops. . . . . . . . . . . . . . . 40

trigonus, Lactophrys. . . . . . . . . . . . 49

Trinectes.................... 47

triporiceps, Conger............... 19

triqueter, Lactophrys. . . . . . . . . . . . 49

triserialis, Ophichthus............. 20

triseriata, Platyrhinoidis........... 8

trispinosa, Odontopyxis........... 40

Trogloglanis. ................ 19

trutta, Salmo.................. 12

tshawytscha, Oncorhynchus......... 11

tuckeri, Monacanthus. . . . . . . . . . . 49

tunicatus, Liparis. . . . . . . . . . . . 41

turneri, Lycodes. . . . . . . . . . . . . 44

tuscumbia, Etheostoma. . . . . . . . . 28

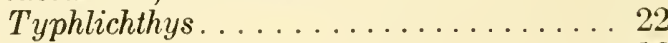

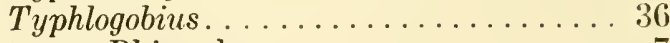

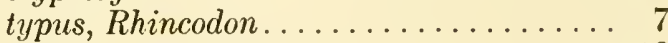

tyrannus, Brevoortia............ 10

\section{$\mathbf{U}$}

Ulvaria.....................44 44

Ulvicola.................... 43

Umbra.................. 12

umbratilis, Notropis............. 16

Umbridae. . . . . . . . . . . . . . . 12

Umbrina..................... 31

umbrosus, Sebastodes............... 38

umpquae, Ptychocheilus. . . . . . . . . 16

uncinatus, Artediellus............. 38

undecimalis, Centropomus. . . . . . . . 25 
undulatus, Menticirrhus

Iicropogon .

unicolor, Hypoplectrus

unicuspis, Ichthyomyzon.

unifasciatus, Hyporhamphus .

uninotatus, Neoclinus

Upeneus

uranidea, Percina

uranops, Phenacobius

Uranoscopidae.

uranoscopus, Notropis

Uraspis.

Urolophus. .

Urophycis

urospilus, Symphurus

usta, Nicholsina.

uter, Cephaloscyllium.

\section{V}

vacca, Rhacochilus

Vacuoqua

valencicnnesi, Moxostoma

variabilis, Eupomacentrus.

variatum, Etheostoma

variegatus, Cyprinodon .

velifer, Carpiodes.

Letharchus.

velox, Euleptorhamphus

venenosa, Mycteroperca. . .

ventralis, Alutera.

Trichopsetta .

Xyrichthys.

ventricosus, Aptocyclus. . . . . . . . . . 41

venustus, Notropis................ 16

Verma..................... 20

vermiculatus, Esox americanus......... 12

verrilli, Lycenchelys. . . . . . . . . . . . . 44

verrucosa, Occa.................40 40

verticalis, Pleuronichthys.......... 47

vespertilio, Ogcocephalus........... 50

vetula, Balistes................ 48

Scarus..................... 34

vetulus, Parophrys.............. 47

vexillaris, Sebastodes.............. 38

vexillarius, Holocentrus. . . . . . . . . . . 24

vicinus, Gymnothorax. . . . . . . . . . . . 19

vigila.x, Pimephales. . . . . . . . . . . 16

villosus, Mallotus................. 12

vinculus, Radulinus............... 40

violacea, Dasyatis................. 9

violaceus, Cebidichthys............. 44

virens, Pollachius................. 22

virescens, Pantosteus. . . . . . . . . . . 18

virgatum, Etheostoma............. 28

virginicus, Anisotremus............ 30

Polydactylus.................. 46

viride, Sparisoma............... 34

viridis, Euleptorhamphus.......... 20
Gymnelis..................44 44

vitreum, Etheostoma. . . . . . . . . . . 28

Stizostedion vitreum . . . . . . . . . . . 29

vittata, Lepidomeda. . . . . . . . . . . . 14

vittatus, Micrognathus............. 23

vivanus, Hemanthias. . . . . . . . . . . . 25

Lutjanus. . . . . . . . . . . . . . . . 26

vivax, Ammocrypta............. 27

volador, Istiophorus-see albicans, $I$.

volitans, Dactylopterus. . . . . . . . . . . 41

Exocoetus.................... 21

volucellus, Notropis............... 16

Vomer......................30

vomer, Selene................. 30

vulpes, Albula............... 10

vulpinus, Alopias.............. 6

vulsa, Stelgis................ 40

W

waccamensis, Fundulus........... 21

warnerensis, Catostomus........... 17

welaka, Notropis. . . . . . . . . . . . 16

welshi, Ophidion. . . . . . . . . . . 45

wheatlandi, Gasterosteus. . . . . . . . 23

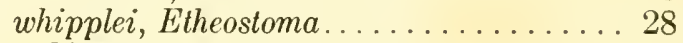

Notropis................... 16

whitehursti, Opisthognathus. . . . . . . 42

williamsoni, Prosopium . . . . . . . . . . 11

witsoni, Sebastodes............... 38

\section{$\mathbf{X}$}

xaenocephalus, Notropis.......... 16

xaenurus, Notropis. ............ 16

xaniurus, Parmaturus............. 7

Xanthichthys................. 49

xanthostigma, Citharichthys..........446

xanthulus, Cynoscion.............. 31

xanthurus, Leiostomus.............. 31

xantusi, Lepidopus. . . . . . . . . . . . 34

xenarcha, Mycteroperca........... 25

Xeneretmus................... 40

xenica, Adinia................ 21

Xenistius.................... 31

Xenopterygii .................. 48

Xererpes................... 43

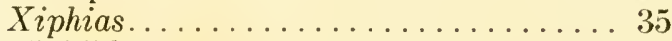

Xiphiidae...................... 35

Xiphister.................... 44

x-punctata, Hybopsis.............. 14

xyosterna, Stellerina............. 40

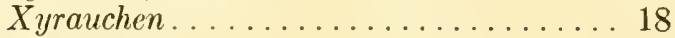

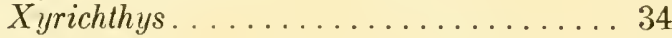

Xystreurys.............. 47

$\mathbf{Y}$

yaito, Euthynnus................. 34

$y$-cauda, Quietula............. 36 
y-graecum, Astroscopus........... 42

yoshiae, Anarchias............... 19

\section{$\mathbf{Z}$}

zacentrus, Sebastodes.............. 38

zachirus, Glyptocephalus. . . . . . . . . 47

Zalembius................... 33

Zalieutes................... 50

Zaniolepidae................... 38

Zaniolepis................... 38

Zaprora................. 45

Zaproridae................ 45

Zapteryx................ 8

zebra, Lythrypnus............... 36

zebrinus, Fundulus. . . . . . . . . . 21

Zeidae.................... 24

Zeiformes................... 24 zenithicus, Coregonus............. 11

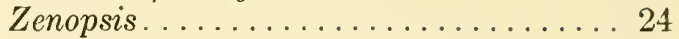

Zeomorphi.................... 24

Zoarcidae................... 44

zonale, Etheostoma.............. 28

zonata, Seriola.................. 30

zonatum, Elassoma.............. 27

zonatus, Chaetodipterus............ 32

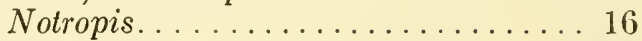

zonifer, Erilepis. . . . . . . . . . . 38

zoniferum, Etheostoma........... 29

zonistius, Notropis. . . . . . . . . . 16

zonope, Jordania . . . . . . . . . . . 39

zophochir, Ophichthus............. 20

zosterae, Hippocampus. . . . . . . . . 23

zygaena, Sphyrna.............. 8

zyopterus, Galeorhinus............. 7 

